\title{
A LAGRANGIAN INTERIOR REGULARITY RESULT FOR THE INCOMPRESSIBLE FREE BOUNDARY EULER EQUATION WITH SURFACE TENSION
}

\author{
MARCELO M. DISCONZI, IGOR KUKAVICA, AND AMJAD TUFFAHA
}

\begin{abstract}
We consider the three-dimensional incompressible free-boundary Euler equations in a bounded domain and with surface tension. Using Lagrangian coordinates, we establish a priori estimates for solutions with minimal regularity assumptions on the initial data.
\end{abstract}

\section{INTRODUCTION}

We consider the free boundary Euler equation of incompressible flow defined on a moving three dimensional domain $\Omega(t) \subseteq \mathbb{R}^{3}$, which read

$$
\begin{aligned}
& u_{t}+(u \cdot \nabla) u+\nabla p=0 \text { in } \mathcal{D} \\
& \operatorname{div} u=0 \text { in } \mathcal{D} \\
& p=\sigma \mathcal{H} \text { on } \partial \mathcal{D} \\
& \left.\left(\partial_{t}+u^{\alpha} \partial_{x_{\alpha}}\right)\right|_{\partial \mathcal{D}} \in T \partial \mathcal{D}
\end{aligned}
$$

where $\mathcal{D}=\bigcup_{0 \leq t \leq T}\{t\} \times \Omega(t), u$ is the fluid's velocity and $p$ its pressure. The symbol $\sigma \geq 0$ denotes the surface tension parameter and $\mathcal{H}$ is, for each $t$, the mean curvature of the boundary $\partial \Omega(t)$ embedded into $\mathbb{R}^{3}$. Also, $T \partial \mathcal{D}$ stands for the tangent bundle of $\partial \mathcal{D}$ and (1.4) expresses the condition that the boundary moves with the speed equal to the normal component of $u$. The initial data are given by

$$
\begin{aligned}
& u(\cdot, 0)=u_{0} \\
& \Omega(0)=\Omega .
\end{aligned}
$$

Our aim in this paper is to obtain a priori estimates for a local-in-time existence result of solutions to this system with minimal regularity assumptions on the initial data and when $\sigma>0$.

The first existence results for (1.1)-(1.6) are those of Nalimov [65] and Yosihara [78], who considered regular irrotational data. In the case of zero surface tension, i.e., $\sigma=0$, Ebin has shown in [36] that the problem is illposed without the Rayleigh-Taylor stability condition. The problem of well-posedness under the Rayleigh-Taylor condition and in the case of zero surface tension was solved by $\mathrm{Wu}[74,75]$. Regarding optimal regularity of the initial data, Wang et al obtained in [73] the local existence under the sharp Sobolev regularity $H^{2.5+\delta}$ for the zero surface tension case, extending the previous result of Alazard et al [6], who considered irrotational data. For the Euler equations in $\mathbb{R}^{2}$ or $\mathbb{R}^{3}$, the sharpness of the exponent $2.5+\delta$ was shown in [15].

The well-posedness of the non-zero surface tension problem, although requiring no additional stability condition, is challenging on its own right and has to be approached differently. While the surface tension has a regularizing effect, the boundary evolution contributes to the energy estimates at top order. Controlling such top order boundary terms, which would automatically vanish in the $\sigma=0$ case, requires an intricate analysis of several boundary terms that express the coupling of the boundary geometry with the interior evolution. Such analysis is particularly delicate in low regularity spaces in that the ellipticity provided by the mean curvature cannot be exploited to same extent as in higher regularity due to the presence of rough coefficients in the mean curvature equation.

MMD is partially supported by the NSF grant DMS-1812826, a Sloan Research Fellowship provided by the Alfred P. Sloan foundation, and from a Discovery grant administered by Vanderbilt University.

IK is partially supported by the NSF grants DMS-1615239 and DMS-1907992. 
Consequently, currently, one does not have estimates that close in spaces near the threshold $H^{2.5+\delta}$ in the case $\sigma>0$, with exception of the simpler situation of irrotational data, for which Alazard, Burq, and Zuily established a full local-wellposedness result with optimal regularity [4].

Regarding rotational fluids with $\sigma>0$, Schweizer [69] constructed solutions with rotational data in $H^{4.5}$ with an additional vorticity condition at the surface. Coutand and Shkoller [25] used the Lagrangian formulation and constructed solutions with $H^{4.5}$ initial data without this restriction. At the same time, Shatah and Zeng obtained in [70] a priori estimates for $H^{3}$ data in Eulerian coordinates using techniques of infinite dimensional geometry in the spirit of Ebin and Marsden [37] (see also [71], where the authors showed how to use their a priori estimates to obtain a local existence result). Ignatova and the second author obtained in [47] a priori estimates with interior regularity in $H^{3.5}$, using the Lagrangian (direct) approach, while Ebin and the first author established a local-existence result in $H^{3.5+\delta}$ using a combination of the Lagrangian approach, infinite-dimensional geometry, and semi-group theory [34].

For other results on irrotational fluids with surface tension see $[2,3,5,11,12,13,17,48,31,41,45,52,79]$. Further related results with non-zero surface tension, including the case of rotational fluids, vortex sheets, twophase fluids, and singular limits, are [22, 27, 32, 33, 39, 51, 66, 68]. Free-boundary problems constitute a very active and fast-growing area of research, and a complete, or even thorough review of prior works is beyond the scope of this paper. A partial list of references relevant to the above discussion and the results of this paper is $[1,7,8,9,10,6,14,18,19,20,21,23,28,26,29,30,40,43,44,46,49,50,56,57,58,60,61,62,63,64,67,76,77]$.

In this manuscript, we use the Lagrangian variables and derive a priori estimates assuming that the initial velocity is in $H^{2.5+\delta}$, where $0<\delta<0.5$. Some further minimal assumptions on the data are also necessary in order to obtain that the second time derivative of the velocity is in $L^{2}$ (cf. Remark 4.3 below).

Unlike in the zero surface tension case, when $\sigma>0$ the interface regularity is driven by the regularity of the pressure, which can be controlled as a solution to an elliptic problem with Neumann boundary condition, in terms of the velocity time derivative. The control of the velocity and its time derivatives is established using a combination of time and tangential energy estimates. Such time and tangential estimates for the velocity lead to some crucial boundary terms whose control is technically challenging (we stress that such boundary terms are absent if $\sigma=0$ ). Exploiting the non-linear structure of the equations and of the boundary condition, we are able to obtain an estimate that reads schematically as

$$
\frac{d}{d t}\left\|\partial_{t}^{2} v\right\|_{L^{2}(\Omega)}^{2}+\frac{d}{d t}\left\|\bar{\partial} \partial_{t}(v \cdot N)\right\|_{L^{2}(\partial \Omega)}^{2}+\frac{d}{d t}\left\|\bar{\partial}^{2+\delta}(v \cdot N)\right\|_{L^{2}(\partial \Omega)}^{2} \lesssim \frac{d}{d t} \int_{\Omega} P_{1}+\frac{d}{d t} \int_{\partial \Omega} P_{2}+P_{3},
$$

where $v$ is the Lagrangian velocity, $P_{1}$ and $P_{2}$ are polynomial expressions on the Lagrangian velocity, the Lagrangian pressure, and their time derivatives, $P_{3}$ is a polynomial in several norms of the fluid variables, $\bar{\partial}$ are derivatives tangent to the boundary, $N$ is the unit outer normal to $\partial \Omega$, and $\delta$ is a small number. Upon time-integration, the term $P_{3}$ is treated by a standard Gronwall argument. The remaining two terms on the right hand side, however, do not have a definite sign. To control such terms we need to show that they can be bounded by lower order terms plus top order terms with small coefficients. Unfortunately, it turns out that this does not seem possible.

However, if we define "non-linear energies" that involve powers of the velocity and its derivatives, we arrive at

$$
\frac{d}{d t}\left\|\partial_{t}^{2} v\right\|_{L^{2}(\Omega)}^{a}+\frac{d}{d t}\left\|\bar{\partial} \partial_{t}(v \cdot N)\right\|_{L^{2}(\partial \Omega)}^{b}+\frac{d}{d t}\left\|\bar{\partial}^{2+\delta}(v \cdot N)\right\|_{L^{2}(\partial \Omega)}^{c} \lesssim \frac{d}{d t} \int_{\Omega} P_{1}+\frac{d}{d t} \int_{\partial \Omega} P_{2}+P_{3},
$$

for certain $a, b, c>0$ (and possibly different $P_{1}, P_{2}$, and $P_{3}$ ). Now, using a combination of interpolation, Sobolev embeddings, and Young's inequality, we obtain that, after time integration, the right hand side is bounded by

$$
\epsilon_{0}\left(\left\|\partial_{t}^{2} v\right\|_{L^{2}(\Omega)}^{\alpha}+\left\|\bar{\partial} \partial_{t}(v \cdot N)\right\|_{L^{2}(\partial \Omega)}^{\beta}+\left\|\bar{\partial}^{2+\delta}(v \cdot N)\right\|_{(\partial \Omega)}^{\gamma}\right)+\int_{0}^{t} P,
$$

where $\epsilon_{0}$ is a small number and $\alpha, \beta$, and $\gamma$ depend on $a, b, c$ and $\delta$. The problem then reduces to the algebraic question of whether it is possible to choose $a, b, c$, so that the powers on both sides match. This turns out to be 
possible ${ }^{1}$ precisely when $0<\delta<0.5$ which, unwrapping all definitions, corresponds to estimating $v$ in $H^{2.5+\delta}$ (this can be seen explicitly in the last estimate, see equations (11.11) and (11.12)). Note that these energies control, aside from $\partial_{t}^{2} v$ in the interior, only tangential derivatives of the normal component of the velocity on the boundary. But once these have been controlled, a bound for the full norms of $v$ is obtained via div-curl estimates, with control of the divergence coming from the divergence-free condition, control of the curl from the Cauchy invariance, and control of the normal components given by the above energy estimate.

To treat the case of a general bounded domain, we employ local coordinates near the boundary and suitably chosen cut-off functions. Such localization techniques are not straightforwardly adapted to the framework of fractional derivatives that we need to employ to obtain estimates in $H^{2.5+\delta}$. Therefore, we consider the problem in two steps. First, we take the initial domain $\Omega$ to have the simpler topology

$$
\Omega=\mathbb{T}^{2} \times[0,1]
$$

and denote its bottom and top boundaries by $\Gamma_{0}$ and $\Gamma_{1}$, respectively. Assume that the lower boundary

$$
\Gamma_{0}=\mathbb{T}^{2} \times\{0\}
$$

is rigid, while the upper boundary $\Gamma_{1}(t)$ evolves in time according to the unknown flow map

$$
\eta\left(x_{1}, x_{2}, 1, t\right): \Gamma_{1} \rightarrow \Gamma_{1}(t)
$$

and is such that $\Gamma_{1}(0)$ equals

$$
\Gamma_{1}=\mathbb{T}^{2} \times\{1\} .
$$

We then establish our result for this type of domains, see Theorem 2.1. This simplified setting already presents all the main difficulties of the problem, but makes it easier to focus on its core aspects without being distracted by the technicalities caused by the use of fractional derivatives in local charts and their interaction with cut-off functions. Then, we show how to adapt the estimates leading to Theorem 2.1 to a general domain, stated as Theorems 12.1 and 12.2 .

\section{THE LAGRANGIAN VARIABLES AND THE MAIN STATEMENT}

We assume that $\Omega(t)$ is initially the 1-periodic channel

$$
\Omega(0)=\Omega=\mathbb{T}^{2} \times[0,1],
$$

with the rigid bottom boundary $\Gamma_{0}=\mathbb{T}^{2} \times\{0\}$. The top boundary $\Gamma_{1}(t)$ evolves and is initially equal to $\Gamma_{1}=$ $\mathbb{T}^{2} \times\{1\}$. (The general case is discussed in Section 12 below.) We use $\eta$ to denote the Lagrangian variable and $a$ the inverse of the matrix $\nabla \eta$. The Lagrangian formulation of the Euler equations then reads

$$
\begin{aligned}
& \partial_{t} v^{\alpha}+a^{\mu \alpha} \partial_{\mu} q=0 \text { in } \Omega \times[0, T] \\
& a^{\alpha \beta} \partial_{\alpha} v_{\beta}=0 \text { in } \Omega \times[0, T] \\
& \partial_{t} \eta=v \text { in } \Omega \times[0, T] \\
& \partial_{t} a^{\alpha \beta}+a^{\alpha \gamma} \partial_{\mu} v_{\gamma} a^{\mu \beta}=0 \text { in } \Omega \times[0, T] \\
& a^{\mu \alpha} N_{\mu} q+\sigma\left|a^{T} N\right| \Delta_{g} \eta^{\alpha}=0 \text { on } \Gamma_{1} \times[0, T] \\
& v^{\mu} N_{\mu}=0 \text { on } \Gamma_{0} \times[0, T],
\end{aligned}
$$

where $N$ is the unit outward normal vector to $\partial \Omega$ and $\Delta_{g}$ is the Laplacian induced on $\partial \Omega(t)$ by $\left.\eta\right|_{\Gamma_{1}}$ i.e.,

$$
\Delta_{g}(\cdot)=\frac{1}{\sqrt{g}} \partial_{i}\left(\sqrt{g} g^{i j} \partial_{j}(\cdot)\right),
$$

where

$$
g_{i j}=\partial_{i} \eta^{\mu} \partial_{j} \eta_{\mu}
$$

\footnotetext{
${ }^{1}$ In the presentation of the results it is not necessary to work with such general $a, b$, and $c$. Having found the correct exponents, we already define our energy with them; see (2.14).
} 
while $g$ is the determinant of the matrix $\left[g_{i j}\right]_{i, j=1,2}$. Above and in the sequel, we use the summation convention on repeated indices. The Greek letters run from 1 to 3, while the Latin go from 1 to 2.

The following is the main statement in which we establish a priori estimates for the local existence of solutions with initial data $v_{0}=\left(v_{0}^{1}, v_{0}^{2}, v_{0}^{3}\right) \in H^{2.5+\epsilon}$, where $\epsilon \in(0,1 / 2)$,

Theorem 2.1. Let $\sigma>0$ and $\epsilon \in(0,1 / 2)$. Assume that $v_{0}$ is a smooth divergence-free vector field on $\Omega$. Then there exist constants $C_{*}, T_{*}>0$ such that any smooth solution $(v, q)$ to (2.2)-(2.7) with initial condition $v_{0}$ defined on the time interval $\left[0, T_{*}\right]$, satisfies

$$
\|v\|_{H^{2.5+\epsilon}}+\left\|\partial_{t} v\right\|_{H^{1.5}}+\left\|\partial_{t}^{2} v\right\|_{L^{2}}+\|q\|_{H^{2.25+\epsilon / 2}}+\left\|\partial_{t} q\right\|_{H^{1}} \leq C_{*},
$$

where $T_{*}, C_{*}>0$ depend only on $\left\|v_{0}\right\|_{H^{2.5+\epsilon}},\left\|\left.v_{0}^{3}\right|_{\Gamma_{1}}\right\|_{H^{2.5}\left(\Gamma_{1}\right)}$, and $\sigma>0$.

Above and in the sequel, if the domain of the Sobolev space is not designated, it is understood to be $\Omega$, while other domains (typically $\Gamma_{1}, \Gamma_{0}$, and $\partial \Omega$ ) are explicitly noted.

In Remark 4.3 below we show that the condition $\left\|\left.v_{0}\right|_{\Gamma_{1}}\right\|_{H^{2.5}\left(\Gamma_{1}\right)}<\infty$ can be replaced by $\left\|\left.\Delta_{2} v_{0}^{3}\right|_{\Gamma_{1}}\right\|_{H^{0.5}\left(\Gamma_{1}\right)}<$ $\infty$, where $\Delta_{2}$ is the boundary Laplacian. This last condition is not only sufficient but is also necessary for $\partial_{t}^{2} v_{0} \in$ $L^{2}$.

Instead of working with $\epsilon>0$, we introduce, for simplicity of notation, the parameter $\nu=1 / 2-\epsilon$ and thus consider

where we assume

$$
v_{0} \in H^{3-\nu}
$$

$$
\nu \in[0,0.5)
$$

By introducing $\nu$, many exponents and Sobolev parameters have simpler forms. Note that we include also the value $\nu=0$ since all the results below hold for this borderline value as well.

The proof consists of a series of estimates on $v$ and $q$ involving the energies

$$
E_{0}=\|v\|_{H^{2.5-\nu / 2}}
$$

and

It is also convenient to introduce the total energy

$$
E_{1}=\left\|\partial_{t} v\right\|_{H^{1.5}}+\left\|\partial_{t}^{2} v\right\|_{L^{2}} .
$$

$$
E=E_{0}^{2}+E_{1}+1
$$

Note that in (2.14) $E_{0}$ is squared while $E_{1}$ is not.

Since $\sigma>0$ does not vary, we set $\sigma=1$ from here on.

As usual, in what follows, the symbol $a \lesssim b$ stands for $a \leq C b$, where $C$ is a constant.

\section{Preliminary estimates}

In the first lemma, we collect a priori estimates on the map $\eta$ and the cofactor matrix $a=(\nabla \eta)^{-1}$.

Lemma 3.1. Assume that $\|v\|_{L^{\infty}\left([0, T] ; H^{3-\nu}(\Omega)\right)} \leq M$. If

$$
T \leq \frac{1}{C M}
$$

where $C \geq 1$ is a sufficiently large constant, then the following statements hold:

(i) $\|\eta\|_{H^{3-\nu}} \leq C$ for $t \in[0, T]$,

(ii) $\|a\|_{H^{2-\nu}} \leq C$,

(iii) $\left\|\partial_{t} a\right\|_{H^{s}} \leq C\|\nabla v\|_{H^{s}}$ for $0 \leq s \leq 2-\nu$ with

$\left\|\partial_{t}^{2} a\right\|_{H^{0.5-\nu / 2}} \leq C\left(\left\|\partial_{t} v\right\|_{H^{1.5-\nu / 2}}+\|v\|_{H^{2}}\|v\|_{H^{2-\nu / 2}}\right)$ and $\left\|\partial_{t}^{2} a\right\|_{H^{\nu / 2}} \leq C\left(\left\|\partial_{t} v\right\|_{H^{1+\nu / 2}}+\|v\|_{H^{2}}\|v\|_{H^{1.5+\nu / 2}}\right)$, and

(iv) For every $\epsilon_{0} \in(0,1]$, we have $\|a-I\|_{H^{2-\nu}} \leq \epsilon_{0}$ and $\|\nabla \eta-I\|_{H^{2-\nu}} \leq \epsilon_{0}$ provided $T \leq 1 / C \epsilon_{0} M$.

Since the proofs follow easily from (2.4) and (2.5), we only briefly outline them. 
Proof of Lemma 3.1. (i) By (2.4), we have

$$
\|\eta\|_{H^{3-\nu}} \leq\|x\|_{H^{3-\nu}}+\left\|\int_{0}^{t} v\right\|_{H^{3-\nu}} \lesssim 1+T M
$$

and the rest follows from the choice (3.1).

(ii) From (2.5), we get $\|a\|_{H^{2-\nu}} \lesssim 1+M \int_{0}^{t}\|a\|_{H^{2-\nu}}^{2}$, and the claim is obtained using the Gronwall lemma.

(iii) Follows directly from (2.5).

(iv) To obtain the claim, we use $a-I=\int_{0}^{t} \partial_{t} a$ and then apply (ii) to obtain

$$
\|a-I\|_{H^{2-\nu}} \lesssim \int_{0}^{t}\left\|\partial_{t} a\right\|_{H^{2-\nu}} \lesssim \int_{0}^{t}\|\nabla v\|_{H^{2-\nu}} \lesssim \epsilon_{0}
$$

for $t \leq T^{\prime}=\epsilon / C M$. Similarly,

$$
\|\nabla \eta-I\|_{H^{2-\nu}} \lesssim \int_{0}^{t}\|\nabla v\|_{H^{2-\nu}} \lesssim \int_{0}^{t}\|v\|_{H^{3-\nu}} \lesssim \epsilon_{0}
$$

under the condition $t \leq \epsilon_{0} / C M$.

\section{Pressure estimates}

For reference, we state the trace inequality for the vector fields with the square integrable divergence (cf. [24, 72]).

Lemma 4.1. Let $\phi$ be a $3 D$ vector field in $L^{2}(\Omega)$, and a $(x)$ a matrix function with components $a^{\mu \alpha} \in L^{\infty}(\Omega)$. If $\partial_{\mu}\left(a^{\mu \alpha} \phi_{\alpha}\right) \in L^{2}(\Omega)$ and $a^{\mu \alpha} \phi_{\alpha} \in L^{2}(\Omega)$ for $\mu=1,2,3$, then $a^{\mu \alpha} \phi_{\alpha} N_{\mu} \in H^{-1 / 2}(\partial \Omega)$ and

$$
\left\|a^{\mu \alpha} \phi_{\alpha} N_{\mu}\right\|_{H^{-1 / 2}(\partial \Omega)} \lesssim\left\|\partial_{\mu}\left(a^{\mu \alpha} \phi_{\alpha}\right)\right\|_{L^{2}(\Omega)}+\sum_{\mu=1}^{3}\left\|a^{\mu \alpha} \phi_{\alpha}\right\|_{L^{2}(\Omega)} .
$$

Next, we derive elliptic estimates satisfied by the Lagrangian pressure $q$ and its time derivative $\partial_{t} q$.

Lemma 4.2. (i) For the Lagrangian pressure q, we have

$$
\|q\|_{H^{2.5-\nu / 2}} \lesssim\|v\|_{H^{1.5}}\|v\|_{H^{2.5-\nu / 2}}+\left\|\partial_{t} v\right\|_{H^{1.5}}^{1-\nu / 3}\left\|\partial_{t} v\right\|_{L^{2}}^{\nu / 3}+\|q(0)\|_{H^{1}}+1+\int_{0}^{t}\left\|\partial_{t} q\right\|_{H^{1}} .
$$

(ii) For the time derivative of the Lagrangian pressure, we have

$$
\begin{aligned}
\left\|\partial_{t} q\right\|_{H^{1}} \lesssim \| & \partial_{t}^{2} v\left\|_{L^{2}}+\right\| \partial_{t} v\left\|_{L^{2}}^{(1-\nu) / 3}\right\| \partial_{t} v\left\|_{H^{1.5}}^{(2+\nu) / 3}\right\| v\left\|_{H^{2.5-\nu / 2}}+\right\| v\left\|_{H^{1.5}}^{(3-3) /(2-\nu)}\right\| v \|_{H^{2.5-\nu / 2}}^{3 /(2-\nu)} \\
& +\|v\|_{H^{2.5-\nu / 2}}\|q\|_{H^{1}}^{\left(2-2 \nu-2 \delta_{0}\right) /(3-\nu)}\|q\|_{H^{2.5-\nu / 2}}^{\left(1+\nu+2 \delta_{0}\right) /(3-\nu)} \\
& +\|v\|_{H^{3-\nu}}^{\nu /(1-\nu)}\|v\|_{H^{2.5-\nu / 2}}^{(1-2 \nu) /(1-\nu)}\left(\|q\|_{H^{1}}+1\right) .
\end{aligned}
$$

Above and in the sequel, $\delta_{0}>0$ denotes an arbitrarily small constant. In most places it appears when bounding the $L^{\infty}$ norm of a quantity with a suitable Sobolev norm.

The exponent $2.5-\nu / 2$ in (4.1) is not the highest regularity of the pressure one may obtain (which is $3-\nu$ ). It is chosen because it is the highest Sobolev exponent for $q$ which can be estimated in terms of $\|v\|_{H^{2.5-\nu / 2}}$, for which in turn we have control based on Section 7 and the properties (9.1) and (10.4) below.

Using the notation (2.12) and (2.13) and introducing

$$
F=\|v\|_{H^{3-\nu}}
$$

we may rewrite (4.1) and (4.2) in simpler forms as

$$
G=\|q\|_{H^{2.5-\nu / 2}} \lesssim P_{0}+\left(P_{0}+\int_{0}^{t} P\right)\left(E_{0}+E_{1}^{1-\nu / 3}\right)+\int_{0}^{t} P
$$


and

$$
\begin{aligned}
H=\left\|\partial_{t} q\right\|_{H^{1}} \lesssim E_{1} & +\left(P_{0}+\int_{0}^{t} P\right) \\
& \times\left(E_{1}^{(2+\nu) / 3} E_{0}+E_{0}^{3 /(2-\nu)}+E_{0} G^{\left(1+\nu+2 \delta_{0}\right) /(3-\nu)}+F^{\nu /(1-\nu)} E_{0}^{(1-2 \nu) /(1-\nu)}\right) .
\end{aligned}
$$

Above and in the sequel, $P_{0}$ denotes a generic polynomial in $\left\|v_{0}\right\|_{H^{3-\nu}},\left\|\partial_{t} v(0)\right\|_{H^{1.5}}$, and $\left\|\partial_{t}^{2} v(0)\right\|_{L^{2}}$, while $P$ denotes a generic polynomial in $\|v\|_{H^{3-\nu}},\left\|\partial_{t} v\right\|_{H^{1.5}},\|q\|_{H^{2.5-\nu / 2}},\left\|\partial_{t} q\right\|_{H^{1}}$, and $\left\|\partial_{t}^{2} v\right\|_{L^{2}}$. Using the notation (2.14) and $\nu<1 / 2$, we then have

$$
G \lesssim\left(P_{0}+\int_{0}^{t} P\right) E^{(3-\nu) / 3}
$$

and

$$
\begin{aligned}
H \lesssim E+\left(P_{0}+\right. & \left.\int_{0}^{t} P\right) \\
& \times\left(E^{(7+2 \nu) / 6}+E^{3 /(4-2 \nu)}+E^{1 / 2} G^{\left(1+\nu+2 \delta_{0}\right) /(3-\nu)}+F^{\nu /(1-\nu)} E^{(1-2 \nu) /(2-2 \nu)}\right) .
\end{aligned}
$$

Since $(7+2 \nu) / 6 \geq 3 /(4-2 \nu)$, we get

$$
H \lesssim E+\left(P_{0}+\int_{0}^{t} P\right)\left(E^{(7+2 \nu) / 6}+E^{1 / 2} G^{\left(1+\nu+2 \delta_{0}\right) /(3-\nu)}+F^{\nu /(1-\nu)} E^{(1-2 \nu) /(2-2 \nu)}\right),
$$

where, as pointed out above, $\delta_{0}>0$ denotes an arbitrarily small constant.

Before the proof of the lemma, we recall the Piola identity

$$
\partial_{\mu} a^{\mu \alpha}=0
$$

(cf. [38, p. 462]).

Proof of Lemma 4.2. First, we apply $a^{\lambda \alpha} \partial_{\lambda}$ to the equation (2.2) and obtain

$$
a^{\lambda \alpha} \partial_{\lambda}\left(a^{\mu}{ }_{\alpha} \partial_{\mu} q\right)=-a^{\lambda \alpha} \partial_{\lambda} \partial_{t} v_{\alpha}=\partial_{t} a^{\lambda \alpha} \partial_{\lambda} v_{\alpha},
$$

where we used the divergence free condition (2.3) in the last step. Isolating $\Delta q$, we obtain the Poisson equation

$$
\begin{aligned}
\Delta q & =\partial_{t} a^{\lambda \alpha} \partial_{\lambda} v_{\alpha}+\left(\delta^{\lambda \alpha}-a^{\lambda \alpha}\right) \partial_{\lambda}\left(\delta_{\alpha}^{\mu} \partial_{\mu} q\right)+a^{\lambda \alpha} \partial_{\lambda}\left(\left(\delta_{\alpha}^{\mu}-a^{\mu}{ }_{\alpha}\right) \partial_{\mu} q\right) \\
& =\partial_{\lambda}\left(\partial_{t} a^{\lambda \alpha} v_{\alpha}\right)+\partial_{\lambda}\left(\left(\delta^{\lambda \alpha}-a^{\lambda \alpha}\right) \partial_{\alpha} q\right)+\partial_{\lambda}\left(a^{\lambda \alpha}\left(\delta_{\alpha}^{\mu}-a_{\alpha}^{\mu}\right) \partial_{\mu} q\right) \\
& =\partial_{\lambda}\left(\partial_{t} a^{\lambda \alpha} v_{\alpha}+\left(\delta^{\lambda \alpha}-a^{\lambda \alpha}\right) \partial_{\alpha} q+a^{\lambda \alpha}\left(\delta_{\alpha}^{\mu}-a_{\alpha}^{\mu}{ }_{\alpha} q\right)=: \partial_{\lambda} f^{\lambda}\right.
\end{aligned}
$$

on $\Omega$, in addition to the boundary conditions

$$
\partial_{3} q=\left(\delta^{\alpha 3}-a^{\alpha 3}\right) \partial_{\alpha} q-\partial_{t} v^{3}=: h_{1} \text { on } \Gamma_{1}
$$

and

$$
\partial_{3} q=\left(\delta^{\alpha 3}-a^{\alpha 3}\right) \partial_{\alpha} q=: h_{2} \text { on } \Gamma_{0},
$$

which result from restricting (2.2) to $\Gamma_{1}$ and $\Gamma_{0}$, respectively. Moreover, from the boundary condition (2.6), we have

$$
q=\left(1-a^{33}\right) q-\partial_{i}\left(\sqrt{g} g^{i j} \partial_{j} \eta^{3}\right) \text { on } \Gamma_{1} \times[0, T] .
$$

We now invoke the estimate for $q$ from $[47,35]$ whereby

$$
\|q\|_{H^{2.5-\nu / 2}} \lesssim\left\|\partial_{\lambda} f^{\lambda}\right\|_{H^{0.5-\nu / 2}}+\left\|h_{1}\right\|_{H^{1-\nu / 2}\left(\Gamma_{1}\right)}+\left\|h_{2}\right\|_{H^{1-\nu / 2}\left(\Gamma_{0}\right)}+\|q\|_{L^{2}\left(\Gamma_{1}\right)} .
$$


Note that

$$
\begin{aligned}
& \left\|\partial_{\lambda} f^{\lambda}\right\|_{H^{0.5-\nu / 2}} \lesssim\left\|\partial_{t} a^{\lambda \alpha} \partial_{\lambda} v_{\alpha}\right\|_{H^{0.5-\nu / 2}}+\left\|\left(\delta^{\lambda \alpha}-a^{\lambda \alpha}\right) \partial_{\alpha} \partial_{\lambda} q\right\|_{H^{0.5-\nu / 2}} \\
& +\left\|a^{\lambda \alpha} \partial_{\lambda} a_{\alpha}^{\mu} \partial_{\mu} q\right\|_{H^{0.5-\nu / 2}}+\left\|a^{\lambda \alpha}\left(\delta_{\alpha}^{\mu}-a_{\alpha}^{\mu}\right) \partial_{\lambda} \partial_{\mu} q\right\|_{H^{0.5-\nu / 2}} \\
& \lesssim\left\|\partial_{t} a^{\lambda \alpha} \partial_{\lambda} v_{\alpha}\right\|_{H^{0.5-\nu / 2}}+\left\|\delta^{\lambda \alpha}-a^{\lambda \alpha}\right\|_{H^{1.5+\delta_{0}}}\left\|\partial_{\alpha} \partial_{\lambda} q\right\|_{H^{0.5-\nu / 2}} \\
& +\left\|a^{\lambda \alpha}\right\|_{H^{1.5+\delta_{0}}}\left\|\partial_{\lambda} a_{\alpha}^{\mu} \partial_{\mu} q\right\|_{H^{0.5-\nu / 2}}+\left\|a^{\lambda \alpha}\right\|_{H^{1.5+\delta_{0}}}\left\|\delta_{\alpha}^{\mu}-a_{\alpha}^{\mu}\right\|_{H^{1.5+\delta_{0}}}\left\|\partial_{\lambda} \partial_{\mu} q\right\|_{H^{0.5-\nu / 2}} \\
& \lesssim\left\|\partial_{t} a^{\lambda \alpha} \partial_{\lambda} v_{\alpha}\right\|_{H^{0.5-\nu / 2}}+\sum_{\alpha, \lambda} \epsilon_{0}\left\|\partial_{\alpha} \partial_{\lambda} q\right\|_{H^{0.5-\nu / 2}} \\
& +\sum_{\alpha, \lambda}\left\|\partial_{\lambda} a_{\alpha}^{\mu} \partial_{\mu} q\right\|_{H^{0.5-\nu / 2}}+\epsilon_{0} \sum_{\lambda, \mu}\left\|\partial_{\lambda} \partial_{\mu} q\right\|_{H^{0.5-\nu / 2}},
\end{aligned}
$$

where we used a multiplicative Sobolev inequality $\|f g\|_{H^{r}} \lesssim\|f\|_{H^{1.5+\delta_{0}}}\|g\|_{H^{r}}$ for $0 \leq r \leq 1.5$. Also, $\epsilon_{0}>0$ denotes everywhere a constant which can be made arbitrarily small by choosing $T>0$ sufficiently small as in Lemma 3.1(iv) above. Therefore,

$$
\left\|\partial_{\lambda} f^{\lambda}\right\|_{H^{0.5-\nu / 2}} \lesssim\left\|\partial_{t} a\right\|_{H^{1}}\|v\|_{H^{2-\nu / 2}}+\epsilon_{0}\|q\|_{H^{2.5-\nu / 2}}+\|a\|_{H^{2-\nu}}\|q\|_{H^{2+\nu / 2}} .
$$

Using (4.10) with (4.7)-(4.9), we get

$$
\begin{aligned}
\|q\|_{H^{2.5-\nu / 2}} \lesssim \| & \partial_{t} a\left\|_{H^{1}}\right\| v\left\|_{H^{2-\nu / 2}}+\epsilon_{0}\right\| q\left\|_{H^{2.5-\nu / 2}}+\right\| q \|_{H^{2+\nu / 2}} \\
& +\|I-a\|_{H^{1+\delta_{0}\left(\Gamma_{1}\right)}}\|\nabla q\|_{H^{1-\nu / 2}\left(\Gamma_{1}\right)}+\left\|\partial_{t} v\right\|_{H^{1-\nu / 2}\left(\Gamma_{1}\right)}+\|I-a\|_{H^{1+\delta_{0}}\left(\Gamma_{0}\right)}\|\nabla q\|_{H^{1-\nu / 2}\left(\Gamma_{0}\right)} \\
& +\left\|1-a^{33}\right\|_{H^{1+\delta_{0}}\left(\Gamma_{1}\right)}\|q\|_{L^{2}\left(\Gamma_{1}\right)}+\left\|\partial_{i}\left(\sqrt{g} g^{i j} \partial_{j} \eta^{3}\right)\right\|_{L^{2}\left(\Gamma_{1}\right)},
\end{aligned}
$$

whence, by Lemma 3.1 (in particular $\|a-I\|_{H^{1.5+\delta_{0}}} \leq \epsilon_{0}$ ),

$$
\begin{aligned}
\|q\|_{H^{2.5-\nu / 2}} & \lesssim\|v\|_{H^{2}}\|v\|_{H^{2-\nu / 2}}+\epsilon_{0}\|q\|_{H^{2.5-\nu / 2}}+\|q\|_{H^{2+\nu / 2}}+\left\|\partial_{t} v\right\|_{H^{1.5-\nu / 2}}+Q\left(\|D \eta\|_{L^{\infty}\left(\Gamma_{1}\right)}\right)\|\eta\|_{H^{2}\left(\Gamma_{1}\right)} \\
& \lesssim\|v\|_{H^{1.5}}\|v\|_{H^{2.5-\nu / 2}}+\epsilon_{0}\|q\|_{H^{2.5-\nu / 2}}+\|q\|_{H^{2+\nu / 2}} \\
& +\left\|\partial_{t} v\right\|_{H^{1.5}}^{1-\nu / 3}\left\|\partial_{t} v\right\|_{L^{2}}^{\nu / 3}+Q\left(\|D \eta\|_{L^{\infty}\left(\Gamma_{1}\right)}\right)\|\eta\|_{H^{2}\left(\Gamma_{1}\right)}
\end{aligned}
$$

where $Q$ denotes a rational function in the indicated argument and where we used

$$
\|v\|_{H^{2}} \lesssim\|v\|_{H^{1.5}}^{(1-\nu) /(2-\nu)}\|v\|_{H^{2.5-\nu / 2}}^{1 /(2-\nu)}
$$

and $\|v\|_{H^{2-\nu / 2}} \lesssim\|v\|_{H^{1.5}}^{1 /(2-\nu)}\|v\|_{H^{2.5-\nu / 2}}^{(1-\nu) /(2-\nu)}$ in the last step. Finally, note that $Q\left(\|D \eta\|_{L^{\infty}\left(\Gamma_{1}\right)}\right)\|\eta\|_{H^{2}\left(\Gamma_{1}\right)} \lesssim 1$ and

$$
\|q\|_{H^{2+\nu / 2}} \leq \epsilon_{0}\|q\|_{H^{2.5-\nu / 2}}+C\|q\|_{H^{1}} \leq \epsilon_{0}\|q\|_{H^{2.5-\nu / 2}}+C\|q(0)\|_{H^{1}}+C \int_{0}^{t}\left\|\partial_{t} q\right\|_{H^{1}} .
$$

(ii) Differentiating $\partial_{\lambda}\left(a^{\lambda \alpha} a^{\mu}{ }_{\alpha} \partial_{\mu} q\right)=\partial_{\lambda}\left(\partial_{t} a^{\lambda \alpha} v_{\alpha}\right)$, we obtain that the time derivative of the Lagrangian pressure satisfies

$$
\begin{aligned}
\partial_{\lambda}\left(a^{\lambda \alpha} a_{\alpha}^{\mu} \partial_{\mu} \partial_{t} q\right)= & \partial_{\lambda}\left(\partial_{t}^{2} a^{\lambda \alpha} v_{\alpha}\right)+\partial_{\lambda}\left(\partial_{t} a^{\lambda \alpha} \partial_{t} v_{\alpha}\right)-\partial_{\lambda}\left(\partial_{t} a^{\lambda \alpha} \partial_{\alpha} q\right) \\
& +\partial_{\lambda}\left(\partial_{t} a^{\lambda \alpha}\left(\delta_{\alpha}^{\mu}-a^{\mu}{ }_{\alpha}\right) \partial_{\mu} q\right)-\partial_{\lambda}\left(a^{\lambda \alpha} \partial_{t} a^{\mu}{ }_{\alpha} \partial_{\mu} q\right)=: \partial_{\lambda} \tilde{f}^{\lambda}
\end{aligned}
$$

in $\Omega$. The boundary conditions, which are deduced from (2.2) and (4.9), read

$$
a^{3 \alpha} a^{\mu}{ }_{\alpha} \partial_{\mu} \partial_{t} q=-a^{3 \alpha} \partial_{t}^{2} v_{\alpha}-\partial_{t} a^{3 \alpha} \partial_{t} v_{\alpha}-\partial_{t}\left(a^{3 \alpha} a^{\mu}{ }_{\alpha}\right) \partial_{\mu} q=: \tilde{h}_{1} \text { on } \Gamma_{1}
$$

and

$$
a^{3 \alpha} a_{\alpha}^{\mu} \partial_{\mu} \partial_{t} q=-a^{3 \alpha} \partial_{t}^{2} v_{\alpha}-\partial_{t} a^{3 \alpha} \partial_{t} v_{\alpha}-\partial_{t}\left(a^{3 \alpha} a_{\alpha}^{\mu}\right) \partial_{\mu} q=: \tilde{h}_{2} \text { on } \Gamma_{0}
$$

with

$$
\partial_{t} q=\partial_{t}\left(1-a^{33}\right) q+\left(1-a^{33}\right) \partial_{t} q-\partial_{i} \partial_{t}\left(\sqrt{g} g^{i j} \partial_{j} \eta^{3}\right) \text { on } \Gamma_{1} \times[0, T] .
$$

Thus we may invoke the estimate

$$
\left\|\partial_{t} q\right\|_{H^{1}} \lesssim\|\tilde{f}\|_{L^{2}}+\left\|\tilde{h}_{1}\right\|_{H^{-1 / 2}\left(\Gamma_{1}\right)}+\left\|\tilde{h}_{2}\right\|_{H^{-1 / 2}\left(\Gamma_{0}\right)}+\left\|\partial_{t} q\right\|_{L^{2}\left(\Gamma_{1}\right)}
$$


from [47] and obtain

$$
\begin{aligned}
\left\|\partial_{t} q\right\|_{H^{1}} \lesssim \sum_{\lambda} & \left(\left\|\partial_{t}^{2} a^{\lambda \alpha} v_{\alpha}\right\|_{L^{2}}+\left\|\partial_{t} a^{\lambda \alpha} \partial_{t} v_{\alpha}\right\|_{L^{2}}+\left\|\partial_{t} a^{\lambda \alpha} \partial_{\alpha} q\right\|_{L^{2}}\right. \\
& \left.+\left\|\partial_{t} a^{\lambda \alpha}\left(\delta_{\alpha}^{\mu}-a^{\mu}{ }_{\alpha}\right) \partial_{\mu} q\right\|_{L^{2}}+\left\|a^{\lambda \alpha} \partial_{t} a^{\mu}{ }_{\alpha} \partial_{\mu} q\right\|_{L^{2}}\right) \\
& +\left\|a^{3 \alpha} \partial_{t}^{2} v_{\alpha}\right\|_{H^{-1 / 2}\left(\Gamma_{1}\right)}+\left\|\partial_{t} a^{3 \alpha} \partial_{t} v_{\alpha}\right\|_{H^{-1 / 2}\left(\Gamma_{1}\right)}+\left\|\partial_{t}\left(a^{3 \alpha} a^{\mu}{ }_{\alpha}\right) \partial_{\mu} q\right\|_{H^{-1 / 2}\left(\Gamma_{1}\right)} \\
& +\left\|a^{3 \alpha} \partial_{t}^{2} v_{\alpha}\right\|_{H^{-1 / 2}\left(\Gamma_{0}\right)}+\left\|\partial_{t} a^{3 \alpha} \partial_{t} v_{\alpha}\right\|_{H^{-1 / 2}\left(\Gamma_{0}\right)}+\left\|\partial_{t}\left(a^{3 \alpha} a^{\mu}{ }_{\alpha}\right) \partial_{\mu} q\right\|_{H^{-1 / 2}\left(\Gamma_{0}\right)} \\
& +\left\|\partial_{t} a^{33} q\right\|_{L^{2}\left(\Gamma_{1}\right)}+\left\|\partial_{i}\left(\partial_{t}\left(\sqrt{g} g^{i j} \partial_{j} \eta^{3}\right)\right)\right\|_{L^{2}\left(\Gamma_{1}\right)} .
\end{aligned}
$$

Denote by $S$ the sum in $\lambda$. Then

$$
S \lesssim\left\|\partial_{t}^{2} a\right\|_{H^{\nu / 2}}\|v\|_{H^{1.5-\nu / 2}}+\left\|\partial_{t} a\right\|_{H^{1.5-\nu / 2}}\left\|\partial_{t} v\right\|_{H^{\nu / 2}}+\left\|\partial_{t} a\right\|_{H^{1.5-\nu / 2}}\|\nabla q\|_{H^{\nu / 2}} .
$$

It turns out that all three terms on the right side of (4.14) appear in the upper bounds (4.15) and (4.16) below thus not leading to any additional terms compared to (4.15) and (4.16). Next, we estimate $\left\|\tilde{h}_{1}\right\|_{H^{-1 / 2}\left(\Gamma_{1}\right)}$ (the bound for $\tilde{h}_{2}$ is the same). We write

$$
\begin{aligned}
\left\|\tilde{h}_{1}\right\|_{H^{-1 / 2}\left(\Gamma_{1}\right)} & \lesssim\left\|a^{3 \alpha} \partial_{t}^{2} v_{\alpha}\right\|_{H^{-1 / 2}\left(\Gamma_{1}\right)}+\left\|\partial_{t} a^{3 \alpha} \partial_{t} v_{\alpha}\right\|_{H^{-1 / 2}\left(\Gamma_{1}\right)}+\left\|\partial_{t}\left(a^{3 \alpha} a_{\alpha}^{\mu}\right) \partial_{\mu} q\right\|_{H^{-1 / 2}\left(\Gamma_{1}\right)} \\
& =T_{1}+T_{2}+T_{3} .
\end{aligned}
$$

For the first term, we have

$$
\begin{aligned}
T_{1} & \lesssim \sum_{\beta}\left\|a^{\beta \alpha} \partial_{t}^{2} v_{\alpha}\right\|_{L^{2}}+\left\|\partial_{\beta}\left(a^{\beta \alpha} \partial_{t}^{2} v_{\alpha}\right)\right\|_{L^{2}}=\sum_{\beta}\left\|a^{\beta \alpha} \partial_{t}^{2} v_{\alpha}\right\|_{L^{2}}+\left\|a^{\beta \alpha} \partial_{\beta} \partial_{t}^{2} v_{\alpha}\right\|_{L^{2}} \\
& \lesssim\|a\|_{L^{\infty}}\left\|\partial_{t}^{2} v\right\|_{L^{2}}+\left\|a^{\beta \alpha} \partial_{\beta} \partial_{t}^{2} v_{\alpha}\right\|_{L^{2}} \lesssim\left\|\partial_{t}^{2} v\right\|_{L^{2}}+\left\|\partial_{t}^{2} a^{\beta \alpha} \partial_{\beta} v_{\alpha}\right\|_{L^{2}}+\left\|\partial_{t} a^{\beta \alpha} \partial_{\beta} \partial_{t} v_{\alpha}\right\|_{L^{2}} \\
& \lesssim\left\|\partial_{t}^{2} v\right\|_{L^{2}}+\left\|\partial_{t}^{2} a\right\|_{H^{\nu / 2}}\|v\|_{H^{2.5-\nu / 2}}+\left\|\partial_{t} a\right\|_{H^{1.5-\nu / 2}}\left\|\partial_{t} v\right\|_{H^{1+\nu / 2}} \\
& \lesssim\left\|\partial_{t}^{2} v\right\|_{L^{2}}+\left\|\partial_{t} v\right\|_{H^{1+\nu / 2}}\|v\|_{H^{2.5-\nu / 2}}+\|v\|_{H^{2}}\|v\|_{H^{1.5+\nu / 2}}\|v\|_{H^{2.5-\nu / 2}}+\|v\|_{H^{2.5-\nu / 2}}\left\|\partial_{t} v\right\|_{H^{1+\nu / 2}} \\
& \lesssim\left\|\partial_{t}^{2} v\right\|_{L^{2}}+\left\|\partial_{t} v\right\|_{L^{2}}^{(1-\nu) / 3}\left\|\partial_{t} v\right\|_{H^{1.5}}^{(2+\nu) / 3}\|v\|_{H^{2.5-\nu / 2}}+\|v\|_{H^{1.5}}^{(3-3 \nu /(2-\nu)}\|v\|_{H^{2.5-\nu / 2}}^{3 /(2-\nu)},
\end{aligned}
$$

where we used Lemma 4.1 in the first step and the divergence condition (2.3) in the fourth. Also, we used (4.11) and $\|v\|_{H^{1.5+\nu / 2}} \lesssim\|v\|_{H^{1.5}}^{(2-2 \nu) /(2-\nu)}\|v\|_{H^{2.5-\nu / 2}}^{\nu /(2-\nu)}$. For $T_{2}$, we apply Lemma 4.1 and estimate

$$
\begin{aligned}
T_{2} & \lesssim\left\|\partial_{\beta}\left(\partial_{t} a^{\beta \alpha} \partial_{t} v_{\alpha}\right)\right\|_{L^{2}}+\sum_{\beta}\left\|\partial_{t} a^{\beta \alpha} \partial_{t} v_{\alpha}\right\|_{L^{2}}=\left\|\partial_{t} a^{\beta \alpha} \partial_{\beta} \partial_{t} v_{\alpha}\right\|_{L^{2}}+\sum_{\beta}\left\|\partial_{t} a^{\beta \alpha} \partial_{t} v_{\alpha}\right\|_{L^{2}} \\
& \lesssim\left\|\partial_{t} a\right\|_{H^{1.5-\nu / 2}}\left\|\partial_{t} v\right\|_{H^{1+\nu / 2}} .
\end{aligned}
$$

Observe that this upper bound already appears in (4.15). For $T_{3}$, we simply use multiplicative Sobolev inequalities to write

$$
T_{3} \lesssim\left\|\partial_{t}\left(a^{3 \alpha} a_{\alpha}^{\mu}\right) \partial_{\mu} q\right\|_{H^{0.5+\delta_{0}}} \lesssim\left\|\partial_{t} a\right\|_{H^{1.5-\nu / 2}}\|q\|_{H^{1.5+\nu / 2+\delta_{0}}}
$$

for an arbitrarily small parameter $\delta_{0}>0$. Therefore,

$$
\begin{gathered}
T_{1}+T_{2}+T_{3} \lesssim\left\|\partial_{t}^{2} v\right\|_{L^{2}}+\left\|\partial_{t} v\right\|_{L^{2}}^{(1-\nu) / 3}\left\|\partial_{t} v\right\|_{H^{1.5}}^{(2+\nu) / 3}\|v\|_{H^{2.5-\nu / 2}}+\|v\|_{H^{1.5}}^{(3-3 \nu) /(2-\nu)}\|v\|_{H^{2.5-\nu / 2}}^{3 /(2-\nu)} \\
+\|v\|_{H^{2.5-\nu / 2}}\|q\|_{H^{1}}^{\left(2-2 \nu-2 \delta_{0}\right) /(3-\nu)}\|q\|_{H^{2.5-\nu / 2}}^{\left(1+\nu+2 \delta_{0}\right) /(3-\nu)} .
\end{gathered}
$$

Finally, we estimate the last two terms in (4.13), representing an upper bound for $\left\|\partial_{t} q\right\|_{L^{2}\left(\Gamma_{1}\right)}$. In this case, we have

$$
\begin{aligned}
\left\|\partial_{t} q\right\|_{L^{2}\left(\Gamma_{1}\right)} & \lesssim\left\|\partial_{t} a^{33} q\right\|_{H^{0.5+\delta_{0}}}+\sum_{i=1}^{2}\left\|\partial_{t}\left(\sqrt{g} g^{i j} \partial_{j} \eta^{3}\right)\right\|_{H^{1.5}} \\
& \lesssim\left\|\partial_{t} a\right\|_{H^{1.5}}\|q\|_{H^{1}}+\|v\|_{H^{2.5}} \lesssim\|v\|_{H^{3-\nu}}^{\nu /(1-\nu)}\|v\|_{H^{2.5-\nu / 2}}^{(1-2 \nu) /(1-\nu)}\left(\|q\|_{H^{1}}+1\right)
\end{aligned}
$$

where we used

$$
\|v\|_{H^{2.5}} \lesssim\|v\|_{H^{3-\nu}}^{\nu /(1-\nu)}\|v\|_{H^{2.5-\nu / 2}}^{(1-2 \nu) /(1-\nu)}
$$


Combining (4.13), (4.14) (cf. the comment right after), (4.17), and (4.18) then leads to (4.2).

Remark 4.3. Here we sketch an argument showing finiteness of the energy $E(0)$ under given conditions on the initial data. First, by (2.2), (2.6), and (4.6), we have

$$
\begin{aligned}
& \Delta q(0)=-\partial^{\alpha} v^{\lambda} \partial_{\lambda} v_{\alpha}(0) \text { in } \Omega \\
& q(0)=-\Delta_{2} \eta^{3}(0)=0 \text { on } \Gamma_{1} \\
& \partial_{3} q=0 \text { on } \Gamma_{0}
\end{aligned}
$$

implying $q(0) \in H^{4-\nu}$ and thus, by (2.2), $\partial_{t} v(0) \in H^{3-\nu}$. Now, based on (4.12), evaluated at $t=0$, we have

$$
\begin{gathered}
\Delta \partial_{t} q(0)=\partial_{t}^{2} a^{\lambda \alpha}(0) \partial_{\lambda} v_{\alpha}(0)+\partial_{t} a^{\lambda \alpha}(0) \partial_{t} \partial_{\lambda} v_{\alpha}(0)-\partial_{t} a^{\lambda \alpha}(0) \partial_{\alpha} \partial_{\lambda} q(0) \\
-\partial_{\lambda} \partial_{t} a^{\mu \lambda}(0) \partial_{\mu} q(0)-\partial_{t} a^{\mu \lambda}(0) \partial_{\mu} \partial_{\lambda} q(0) \in H^{2-\nu},
\end{gathered}
$$

with the boundary conditions

$$
\partial_{t} q(0)=\partial_{3} v_{3}(0) q(0)-\Delta_{2} v^{3}(0) \text { on } \Gamma_{1}
$$

and

$$
\partial_{3} \partial_{t} q(0)=-\partial_{t} a^{\mu 3} \partial_{\mu} q(0) \text { on } \Gamma_{0}
$$

which follow from (2.6) and (2.2) respectively. Note that $\partial_{t} a(0)=-\nabla v(0) \in H^{2-\nu}$ and $\partial_{t}^{2} a(0)=-\partial_{t} \nabla v(0)+$ $\nabla v(0) \nabla v(0) \in H^{2-\nu}$, from where, using

$$
\left.\Delta_{2} v^{3}(0)\right|_{\Gamma_{1}} \in H^{1 / 2}\left(\Gamma_{1}\right)
$$

which in turn follows from $\left.v(0)\right|_{\Gamma_{1}} \in H^{2.5}\left(\Gamma_{1}\right)$, we get $\partial_{t} q(0) \in H^{1}$, from where $\partial_{t}^{2} v(0) \in L^{2}(\Omega)$.

As pointed out above, the condition (4.20) is not only sufficient, but also necessary for $\partial_{t}^{2} v(0) \in L^{2}(\Omega)$. To show this, assume that $\partial_{t}^{2} v(0) \in L^{2}(\Omega)$. Then $\partial_{t} q(0) \in H^{1}$ implying $\left.\partial_{t} q(0)\right|_{\Gamma_{1}} \in H^{1 / 2}\left(\Gamma_{1}\right)$. Using (4.19), we get that (4.20) holds.

\section{A COFACTOR TYPE CANCELLATION}

In the energy estimate on $\partial_{t}^{2} v$, the highest order term is the one where all the derivatives fall on $a$. Thus we need to treat the term

$$
T=\int_{0}^{t} \int_{\Omega} q \mathcal{D} a^{\mu \alpha} \mathcal{D} \partial_{\mu} v_{\alpha}
$$

where $v=\partial_{t} \eta$. Here $\mathcal{D}$ represents a differential operator, commuting with spatial and time derivatives. We shall use this with $\mathcal{D}=\partial_{t}^{2}$. In this section, we rewrite (5.1) using the cofactor form of $a$ and applying cross-integration by parts.

First, note that we have

$$
a^{1 \alpha}=\epsilon^{\alpha \lambda \tau} \partial_{2} \eta_{\lambda} \partial_{3} \eta_{\tau}, a^{2 \alpha}=-\epsilon^{\alpha \lambda \tau} \partial_{1} \eta_{\lambda} \partial_{3} \eta_{\tau}, a^{3 \alpha}=\epsilon^{\alpha \lambda \tau} \partial_{1} \eta_{\lambda} \partial_{2} \eta_{\tau}
$$

and thus, expanding in $\mu$ and using (5.2),

$$
\begin{aligned}
T=\int_{0}^{t} & \int_{\Omega} q \epsilon^{\alpha \lambda \tau} \mathcal{D}\left(\partial_{2} \eta_{\lambda} \partial_{3} \eta_{\tau}\right) \mathcal{D} \partial_{1} v_{\alpha}-\int_{0}^{t} \int_{\Omega} q \epsilon^{\alpha \lambda \tau} \mathcal{D}\left(\partial_{1} \eta_{\lambda} \partial_{3} \eta_{\tau}\right) \mathcal{D} \partial_{2} v_{\alpha} \\
& +\int_{0}^{t} \int_{\Omega} q \epsilon^{\alpha \lambda \tau} \mathcal{D}\left(\partial_{1} \eta_{\lambda} \partial_{2} \eta_{\tau}\right) \mathcal{D} \partial_{3} v_{\alpha}
\end{aligned}
$$


from where

$$
\begin{aligned}
& T=\int_{0}^{t} \int_{\Omega} q \epsilon^{\alpha \lambda \tau} \partial_{2} \mathcal{D} \eta_{\lambda} \partial_{3} \eta_{\tau} \partial_{1} \mathcal{D} v_{\alpha}+\int_{0}^{t} \int_{\Omega} q \epsilon^{\alpha \lambda \tau} \partial_{2} \eta_{\lambda} \partial_{3} \mathcal{D} \eta_{\tau} \partial_{1} \mathcal{D} v_{\alpha} \\
&-\int_{0}^{t} \int_{\Omega} q \epsilon^{\alpha \lambda \tau} \partial_{1} \mathcal{D} \eta_{\lambda} \partial_{3} \eta_{\tau} \partial_{2} \mathcal{D} v_{\alpha}-\int_{0}^{t} \int_{\Omega} q \epsilon^{\alpha \lambda \tau} \partial_{1} \eta_{\lambda} \partial_{3} \mathcal{D} \eta_{\tau} \partial_{2} \mathcal{D} v_{\alpha} \\
&+\int_{0}^{t} \int_{\Omega} q \epsilon^{\alpha \lambda \tau} \partial_{1} \mathcal{D} \eta_{\lambda} \partial_{2} \eta_{\tau} \partial_{3} \mathcal{D} v_{\alpha}+\int_{0}^{t} \int_{\Omega} q \epsilon^{\alpha \lambda \tau} \partial_{1} \eta_{\lambda} \partial_{2} \mathcal{D} \eta_{\tau} \partial_{3} \mathcal{D} v_{\alpha}+L \\
&= T_{1}+\cdots \\
&+T_{6}+L
\end{aligned}
$$

where

$$
\begin{aligned}
L=\int_{0}^{t} & \int_{\Omega} q \epsilon^{\alpha \lambda \tau}\left(\mathcal{D}\left(\partial_{2} \eta_{\lambda} \partial_{3} \eta_{\tau}\right)-\partial_{2} \mathcal{D} \eta_{\lambda} \partial_{3} \eta_{\tau}-\partial_{2} \eta_{\lambda} \partial_{3} \mathcal{D} \eta_{\tau}\right) \mathcal{D} \partial_{1} v_{\alpha} \\
& -\int_{0}^{t} \int_{\Omega} q \epsilon^{\alpha \lambda \tau}\left(\mathcal{D}\left(\partial_{1} \eta_{\lambda} \partial_{3} \eta_{\tau}\right)-\partial_{1} \mathcal{D} \eta_{\lambda} \partial_{3} \eta_{\tau}-\partial_{1} \eta_{\lambda} \partial_{3} \mathcal{D} \eta_{\tau}\right) \mathcal{D} \partial_{2} v_{\alpha} \\
& +\int_{0}^{t} \int_{\Omega} q \epsilon^{\alpha \lambda \tau}\left(\mathcal{D}\left(\partial_{1} \eta_{\lambda} \partial_{2} \eta_{\tau}\right)-\partial_{1} \mathcal{D} \eta_{\lambda} \partial_{2} \eta_{\tau}-\partial_{1} \eta_{\lambda} \partial_{2} \mathcal{D} \eta_{\tau}\right) \mathcal{D} \partial_{3} v_{\alpha}
\end{aligned}
$$

represents the sum of the lower order terms that appear when we distribute $\mathcal{D}$ on the product $\epsilon^{\alpha \lambda \tau} \partial_{\alpha} \eta_{\lambda} \partial_{\beta} \eta_{\tau}$ and all derivatives do not fall on a single $\eta$.

In order to proceed, we need for $\mathcal{D}$ to contain at least one time derivative. Thus we now restrict our attention to

$$
\mathcal{D}=\mathcal{E} \partial_{t}
$$

where $\mathcal{E}$ is a linear differential operator, for which we assume

$$
\left[\mathcal{E}, \partial_{t}\right]=0
$$

and

$$
\left[\mathcal{E}, \partial_{\alpha}\right]=0, \quad \alpha=1,2,3 .
$$

Further below we apply the resulting identity to $\mathcal{E}=\partial_{t}$.

We group the leading terms in (5.3) as

$$
\begin{aligned}
& T_{1}+T_{3}=\int_{0}^{t} \int_{\Omega} q \epsilon^{\alpha \lambda \tau} \partial_{3} \eta_{\tau} \partial_{2} \mathcal{D} \eta_{\lambda} \partial_{1} \mathcal{D} v_{\alpha}-\int_{0}^{t} \int_{\Omega} q \epsilon^{\alpha \lambda \tau} \partial_{3} \eta_{\tau} \partial_{1} \mathcal{D} \eta_{\lambda} \partial_{2} \mathcal{D} v_{\alpha} \\
& T_{2}+T_{5}=\int_{0}^{t} \int_{\Omega} q \epsilon^{\alpha \lambda \tau} \partial_{2} \eta_{\lambda} \partial_{3} \mathcal{D} \eta_{\tau} \partial_{1} \mathcal{D} v_{\alpha}+\int_{0}^{t} \int_{\Omega} q \epsilon^{\alpha \lambda \tau} \partial_{2} \eta_{\tau} \partial_{1} \mathcal{D} \eta_{\lambda} \partial_{3} \mathcal{D} v_{\alpha} \\
& T_{4}+T_{6}=-\int_{0}^{t} \int_{\Omega} q \epsilon^{\alpha \lambda \tau} \partial_{1} \eta_{\lambda} \partial_{3} \mathcal{D} \eta_{\tau} \partial_{2} \mathcal{D} v_{\alpha}+\int_{0}^{t} \int_{\Omega} q \epsilon^{\alpha \lambda \tau} \partial_{1} \eta_{\lambda} \partial_{2} \mathcal{D} \eta_{\tau} \partial_{3} \mathcal{D} v_{\alpha}
\end{aligned}
$$

Here we present the treatment of the sum $T_{2}+T_{5}$; the two other pairs are treated similarly (see below). Thus consider

$$
\begin{aligned}
T_{2}+T_{5}= & \int_{0}^{t} \int_{\Omega} q \epsilon^{\alpha \lambda \tau} \partial_{2} \eta_{\lambda} \partial_{3} \mathcal{E} v_{\tau} \partial_{1} \mathcal{E} \partial_{t} v_{\alpha}+\int_{0}^{t} \int_{\Omega} q \epsilon^{\alpha \lambda \tau} \partial_{2} \eta_{\tau} \partial_{1} \mathcal{E} v_{\lambda} \partial_{3} \mathcal{E} \partial_{t} v_{\alpha} \\
= & \left.\int_{\Omega} q \epsilon^{\alpha \lambda \tau} \partial_{2} \eta_{\lambda} \partial_{3} \mathcal{E} v_{\tau} \partial_{1} \mathcal{E} v_{\alpha}\right|_{0} ^{t}-\int_{0}^{t} \int_{\Omega} q \epsilon^{\alpha \lambda \tau} \partial_{2} \eta_{\lambda} \partial_{t} \partial_{3} \mathcal{E} v_{\tau} \partial_{1} \mathcal{E} v_{\alpha} \\
& \quad-\int_{0}^{t} \int_{\Omega} \partial_{t}\left(q \epsilon^{\alpha \lambda \tau} \partial_{2} \eta_{\lambda}\right) \partial_{3} \mathcal{E} v_{\tau} \partial_{1} \mathcal{E} v_{\alpha}+\int_{0}^{t} \int_{\Omega} q \epsilon^{\alpha \lambda \tau} \partial_{2} \eta_{\tau} \partial_{1} \mathcal{E} v_{\lambda} \partial_{3} \mathcal{E} \partial_{t} v_{\alpha} \\
= & I_{1}+I_{2}+I_{3}+I_{4},
\end{aligned}
$$

where we integrated by parts in $t$ in the first integral. By relabeling the indices, we may rewrite the fourth integral as $I_{4}=\int_{0}^{t} \int_{\Omega} q \epsilon^{\tau \alpha \lambda} \partial_{2} \eta_{\lambda} \partial_{1} \mathcal{E} v_{\alpha} \partial_{3} \mathcal{E} \partial_{t} v_{\tau}=\int_{0}^{t} \int_{\Omega} q \epsilon^{\alpha \lambda \tau} \partial_{2} \eta_{\lambda} \partial_{1} \mathcal{E} v_{\alpha} \partial_{3} \mathcal{E} \partial_{t} v_{\tau}$ and the last expression cancels with $I_{2}$. 
Next we treat the first term on the far side of (5.8) evaluated at $t$ by writing

$$
\left.I_{1}\right|_{t}=\int_{\Omega} q \epsilon^{\alpha \lambda \tau} \partial_{2} \eta_{\lambda} \partial_{3} \mathcal{E} v_{\tau} \partial_{1} \mathcal{E} v_{\alpha}=\int_{\Omega} q \epsilon^{\alpha 2 \tau} \partial_{3} \mathcal{E} v_{\tau} \partial_{1} \mathcal{E} v_{\alpha}+\int_{\Omega} q \epsilon^{\alpha \lambda \tau}\left(\int_{0}^{t} \partial_{2} v_{\lambda}\right) \partial_{3} \mathcal{E} v_{\tau} \partial_{1} \mathcal{E} v_{\alpha}
$$

where we used $\partial_{2} \eta_{\lambda}=\delta_{2 \lambda}+\int_{0}^{t} \partial_{2} v_{\lambda}$ in the last step.

Note that $T_{1}+T_{3}$ is obtained from $T_{2}+T_{5}$ by switching $x_{2}$ and $x_{3}$ and multiplying by -1 , while $T_{4}+T_{6}$ is obtained from $T_{2}+T_{5}$ by switching $x_{1}$ and $x_{2}$ and also multiplying by -1 .

We summarize the above derivation in the following statement.

Lemma 5.1. Consider the integral $T=\int_{\Omega} q \mathcal{E} \partial_{t} a^{\mu \alpha} \mathcal{E} \partial_{t} \partial_{\mu} v_{\alpha}$, where $\mathcal{E}$ is a differential operator which commutes with $\partial_{t}$ and $\partial_{\alpha}$, i.e., (5.6) and (5.7) hold. Then we have

$$
\begin{aligned}
T=\int_{\Omega} & \left.q \epsilon^{\alpha 2 \tau} \partial_{3} \mathcal{E} v_{\tau} \partial_{1} \mathcal{E} v_{\alpha}\right|_{t}-\left.\int_{\Omega} q \epsilon^{\alpha 3 \tau} \partial_{2} \mathcal{E} v_{\tau} \partial_{1} \mathcal{E} v_{\alpha}\right|_{t}-\left.\int_{\Omega} q \epsilon^{\alpha 1 \tau} \partial_{3} \mathcal{E} v_{\tau} \partial_{2} \mathcal{E} v_{\alpha}\right|_{t} \\
& -\int_{0}^{t} \int_{\Omega} \partial_{t}\left(q \epsilon^{\alpha \lambda \tau} \partial_{2} \eta_{\lambda}\right) \partial_{3} \mathcal{E} v_{\tau} \partial_{1} \mathcal{E} v_{\alpha}+\int_{0}^{t} \int_{\Omega} \partial_{t}\left(q \epsilon^{\alpha \lambda \tau} \partial_{3} \eta_{\lambda}\right) \partial_{2} \mathcal{E} v_{\tau} \partial_{1} \mathcal{E} v_{\alpha} \\
& +\int_{0}^{t} \int_{\Omega} \partial_{t}\left(q \epsilon^{\alpha \lambda \tau} \partial_{1} \eta_{\lambda}\right) \partial_{3} \mathcal{E} v_{\tau} \partial_{2} \mathcal{E} v_{\alpha} \\
& +\int_{\Omega} q \epsilon^{\alpha \lambda \tau}\left(\int_{0}^{t} \partial_{2} v_{\lambda}\right) \partial_{3} \mathcal{E} v_{\tau} \partial_{1} \mathcal{E} v_{\alpha}-\int_{\Omega} q \epsilon^{\alpha \lambda \tau}\left(\int_{0}^{t} \partial_{3} v_{\lambda}\right) \partial_{2} \mathcal{E} v_{\tau} \partial_{1} \mathcal{E} v_{\alpha} \\
& -\int_{\Omega} q \epsilon^{\alpha \lambda \tau}\left(\int_{0}^{t} \partial_{1} v_{\lambda}\right) \partial_{3} \mathcal{E} v_{\tau} \partial_{2} \mathcal{E} v_{\alpha} \\
& -\left.\int_{\Omega} q \epsilon^{\alpha 2 \tau} \partial_{3} \mathcal{E} v_{\tau} \partial_{1} \mathcal{E} v_{\alpha}\right|_{0}+\left.\int_{\Omega} q \epsilon^{\alpha 3 \tau} \partial_{2} \mathcal{E} v_{\tau} \partial_{1} \mathcal{E} v_{\alpha}\right|_{0}+\left.\int_{\Omega} q \epsilon^{\alpha 1 \tau} \partial_{3} \mathcal{E} v_{\tau} \partial_{2} \mathcal{E} v_{\alpha}\right|_{0}+L
\end{aligned}
$$

where $L$ is given in (5.4).

It is helpful to expand the commutator term $L$ using (5.5). We thus have

$$
\begin{aligned}
L=\int_{\Omega} & q \epsilon^{\alpha \lambda \tau}\left(\mathcal{E}\left(\partial_{2} v_{\lambda} \partial_{3} \eta_{\tau}\right)-\partial_{2} \mathcal{E} v_{\lambda} \partial_{3} \eta_{\tau}\right) \mathcal{E} \partial_{t} \partial_{1} v_{\alpha}+\int_{\Omega} q \epsilon^{\alpha \lambda \tau}\left(\mathcal{E}\left(\partial_{2} \eta_{\lambda} \partial_{3} v_{\tau}\right)-\partial_{2} \eta_{\lambda} \partial_{3} \mathcal{E} v_{\tau}\right) \mathcal{E} \partial_{t} \partial_{1} v_{\alpha} \\
& -\int_{\Omega} q \epsilon^{\alpha \lambda \tau}\left(\mathcal{E}\left(\partial_{1} v_{\lambda} \partial_{3} \eta_{\tau}\right)-\partial_{1} \mathcal{E} v_{\lambda} \partial_{3} \eta_{\tau}\right) \mathcal{E} \partial_{t} \partial_{2} v_{\alpha}-\int_{\Omega} q \epsilon^{\alpha \lambda \tau}\left(\mathcal{E}\left(\partial_{1} \eta_{\lambda} \partial_{3} v_{\tau}\right)-\partial_{1} \eta_{\lambda} \partial_{3} \mathcal{E} v_{\tau}\right) \mathcal{E} \partial_{t} \partial_{2} v_{\alpha} \\
& \left.+\int_{\Omega} q \epsilon^{\alpha \lambda \tau}\left(\mathcal{E}\left(\partial_{1} v_{\lambda} \partial_{2} \eta_{\tau}\right)-\partial_{1} \mathcal{E} v_{\lambda} \partial_{2} \eta_{\tau}\right)\right) \mathcal{E} \partial_{t} \partial_{3} v_{\alpha}+\int_{\Omega} q \epsilon^{\alpha \lambda \tau}\left(\mathcal{E}\left(\partial_{1} \eta_{\lambda} \partial_{2} v_{\tau}\right)-\partial_{1} \eta_{\lambda} \partial_{2} \mathcal{E} v_{\tau}\right) \mathcal{E} \partial_{t} \partial_{3} v_{\alpha}
\end{aligned}
$$

\section{A BOUNDARY INTEGRAL ESTIMATE}

In Sections 7 and 8, we obtain two integrals of the form $K=-\int_{\Gamma_{1}} \mathcal{E} \partial_{t}\left(a^{\mu \alpha} q\right) \mathcal{E} \partial_{t} v_{\alpha} N_{\mu}=\int_{\Gamma_{1}} \mathcal{E} \partial_{t}\left(\sqrt{g} \Delta_{g} \eta^{\alpha}\right) \mathcal{E} \partial_{t} v_{\alpha}$ ( $I_{4}$ and $J_{4}$ in (7.4) and (8.1) below, respectively), where $\mathcal{E}$ is as in the previous section, i.e., a differential operator which commutes with spatial and time derivatives. Using the identity

$$
\partial_{t}\left(\sqrt{g} \Delta_{g} \eta^{\alpha}\right)=\partial_{i}\left(\sqrt{g} g^{i j}\left(\delta_{\lambda}^{\alpha}-g^{k l} \partial_{k} \eta^{\alpha} \partial_{l} \eta_{\lambda}\right) \partial_{t} \partial_{j} \eta^{\lambda}+\sqrt{g}\left(g^{i j} g^{k l}-g^{l j} g^{i k}\right) \partial_{j} \eta^{\alpha} \partial_{k} \eta_{\lambda} \partial_{t} \partial_{l} \eta^{\lambda}\right)
$$

from [35] and $v=\partial_{t} \eta$, we get

$$
\begin{aligned}
K= & \int_{\Gamma_{1}} \mathcal{E} \partial_{t} v_{\alpha} \mathcal{E} \partial_{i}\left(\sqrt{g} g^{i j}\left(\delta_{\lambda}^{\alpha}-g^{k l} \partial_{k} \eta^{\alpha} \partial_{l} \eta_{\lambda}\right) \partial_{j} v^{\lambda}+\sqrt{g}\left(g^{i j} g^{k l}-g^{l j} g^{i k}\right) \partial_{j} \eta^{\alpha} \partial_{k} \eta_{\lambda} \partial_{l} v^{\lambda}\right) \\
= & -\int_{\Gamma_{1}} \partial_{i} \mathcal{E} \partial_{t} v_{\alpha} \mathcal{E}\left(\sqrt{g} g^{i j}\left(\delta_{\lambda}^{\alpha}-g^{k l} \partial_{k} \eta^{\alpha} \partial_{l} \eta_{\lambda}\right) \partial_{j} v^{\lambda}\right) \\
& \quad-\int_{\Gamma_{1}} \partial_{i} \mathcal{E} \partial_{t} v_{\alpha} \mathcal{E}\left(\sqrt{g}\left(g^{i j} g^{k l}-g^{l j} g^{i k}\right) \partial_{j} \eta^{\alpha} \partial_{k} \eta_{\lambda} \partial_{l} v^{\lambda}\right)=K_{1}+K_{2} .
\end{aligned}
$$


We denote by $\Pi$ the projection onto the normal of the moving boundary, given explicitly by

$$
\Pi_{\lambda}^{\alpha}=\delta_{\lambda}^{\alpha}-g^{k l} \partial_{k} \eta^{\alpha} \partial_{l} \eta_{\lambda} .
$$

In Section 10, we show how estimates on $\Pi v$ (and its time derivatives) yield estimates on the normal component of $v$ (and its time derivatives). Using $\Pi$, we thus have

$$
\begin{aligned}
K_{1} & =-\int_{\Gamma_{1}} \mathcal{E}\left(\sqrt{g} g^{i j} \Pi_{\lambda}^{\alpha} \partial_{j} v^{\lambda}\right) \partial_{i} \mathcal{E} \partial_{t} v_{\alpha} \\
& =-\int_{\Gamma_{1}} \sqrt{g} g^{i j} \Pi_{\lambda}^{\alpha} \mathcal{E} \partial_{j} v^{\lambda} \partial_{i} \mathcal{E} \partial_{t} v_{\alpha}-\int_{\Gamma_{1}}\left(\mathcal{E}\left(\sqrt{g} g^{i j} \Pi_{\lambda}^{\alpha} \partial_{j} v^{\lambda}\right)-\sqrt{g} g^{i j} \Pi_{\lambda}^{\alpha} \mathcal{E} \partial_{j} v^{\lambda}\right) \partial_{i} \mathcal{E} \partial_{t} v_{\alpha}=K_{11}+K_{12} .
\end{aligned}
$$

By $\Pi_{\lambda}^{\alpha}=\Pi_{\mu}^{\alpha} \Pi_{\lambda}^{\mu}$ (cf. [35]), we may rewrite the first term as

$$
\begin{aligned}
K_{11}=- & \int_{\Gamma_{1}} \sqrt{g} g^{i j} \Pi_{\lambda}^{\mu} \partial_{j} \mathcal{E} v^{\lambda} \Pi_{\mu}^{\alpha} \partial_{i} \mathcal{E} \partial_{t} v_{\alpha} \\
=- & \frac{1}{2} \frac{d}{d t} \int_{\Gamma_{1}} \sqrt{g} g^{i j} \Pi_{\lambda}^{\mu} \partial_{j} \mathcal{E} v^{\lambda} \Pi_{\mu}^{\alpha} \partial_{i} \mathcal{E} v_{\alpha}+\frac{1}{2} \int_{\Gamma_{1}} \partial_{t}\left(\sqrt{g} g^{i j} \Pi_{\lambda}^{\alpha}\right) \partial_{j} \mathcal{E} v^{\lambda} \partial_{i} \mathcal{E} v_{\alpha} \\
=- & \frac{1}{2} \frac{d}{d t} \int_{\Gamma_{1}} \sqrt{g} g^{i j} \Pi_{\lambda}^{\mu} \partial_{j} \mathcal{E} v^{\lambda} \Pi_{\mu}^{\alpha} \partial_{i} \mathcal{E} v_{\alpha}+\frac{1}{2} \int_{\Gamma_{1}} \partial_{t}\left(\sqrt{g} g^{i j}\right) \Pi_{\lambda}^{\alpha} \partial_{j} \mathcal{E} v^{\lambda} \partial_{i} \mathcal{E} v_{\alpha} \\
& +\frac{1}{2} \int_{\Gamma_{1}} \sqrt{g} g^{i j} \partial_{t}\left(\Pi_{\lambda}^{\alpha}\right) \partial_{j} \mathcal{E} v^{\lambda} \partial_{i} \mathcal{E} v_{\alpha} .
\end{aligned}
$$

We thus obtain

$$
\begin{aligned}
K_{11} \lesssim- & \frac{1}{2} \frac{d}{d t} \int_{\Gamma_{1}} \sqrt{g} g^{i j} \Pi_{\lambda}^{\mu} \partial_{j} \mathcal{E} v^{\lambda} \Pi_{\mu}^{\alpha} \partial_{i} \mathcal{E} v_{\alpha}+P\left(\|\eta\|_{H^{2.5+\delta_{0}}}\right)\|v\|_{H^{2.5+\delta_{0}}}\|\Pi \bar{\partial} \mathcal{E} v\|_{L^{2}\left(\Gamma_{1}\right)}^{2} \\
& +P\left(\|\eta\|_{H^{2.5+\delta_{0}}}\right)\|v\|_{H^{2.5} \delta_{0}}\|\mathcal{E} v\|_{H^{1.5}}^{2}
\end{aligned}
$$

where

$$
\bar{\partial}=\nabla_{2}=\left(\partial_{1}, \partial_{2}\right)
$$

Next, we consider the second term in (6.1). We have

$$
\begin{aligned}
K_{2}=- & \int_{\Gamma_{1}} \sqrt{g}\left(g^{i j} g^{k l}-g^{l j} g^{i k}\right) \partial_{j} \eta^{\alpha} \partial_{k} \eta_{\lambda} \partial_{l} \mathcal{E} v^{\lambda} \partial_{i} \mathcal{E} \partial_{t} v_{\alpha} \\
& -\int_{\Gamma_{1}}\left(\mathcal{E}\left(\sqrt{g}\left(g^{i j} g^{k l}-g^{l j} g^{i k}\right) \partial_{j} \eta^{\alpha} \partial_{k} \eta_{\lambda} \partial_{l} v^{\lambda}\right)\right. \\
& \left.-\sqrt{g}\left(g^{i j} g^{k l}-g^{l j} g^{i k}\right) \partial_{j} \eta^{\alpha} \partial_{k} \eta_{\lambda} \partial_{l} \mathcal{E} v^{\lambda}\right) \partial_{i} \mathcal{E} \partial_{t} v_{\alpha}=K_{21}+K_{22} .
\end{aligned}
$$

As in [25] (cf. also [35]), we may write $K_{21}=-\int_{\Gamma_{1}} \sqrt{g}^{-1}\left(\partial_{t} \operatorname{det} A^{1}+\operatorname{det} A^{2}+\operatorname{det} A^{3}\right.$ ), where

$$
A^{1}=\left(\begin{array}{ll}
\partial_{1} \eta_{\mu} \partial_{1} \mathcal{E} v^{\mu} & \partial_{1} \eta_{\mu} \partial_{2} \mathcal{E} v^{\mu} \\
\partial_{2} \eta_{\mu} \partial_{1} \mathcal{E} v^{\mu} & \partial_{2} \eta_{\mu} \partial_{2} \mathcal{E} v^{\mu}
\end{array}\right), A^{2}=\left(\begin{array}{ll}
\partial_{1} v_{\mu} \partial_{1} \mathcal{E} v^{\mu} & \partial_{1} \eta_{\mu} \partial_{2} \mathcal{E} v^{\mu} \\
\partial_{2} v_{\mu} \partial_{1} \mathcal{E} v^{\mu} & \partial_{2} \eta_{\mu} \partial_{2} \mathcal{E} v^{\mu}
\end{array}\right), A^{3}=\left(\begin{array}{ll}
\partial_{1} \eta_{\mu} \partial_{1} \mathcal{E} v^{\mu} & \partial_{1} v_{\mu} \partial_{2} \mathcal{E} v^{\mu} \\
\partial_{2} \eta_{\mu} \partial_{1} \mathcal{E} v^{\mu} & \partial_{2} v_{\mu} \partial_{2} \mathcal{E} v^{\mu}
\end{array}\right)
$$

Therefore,

$$
\begin{aligned}
K_{21} & =-\int_{\Gamma_{1}} \partial_{t}\left(\frac{1}{\sqrt{g}} \operatorname{det} A^{1}\right)+\int_{\Gamma_{1}} \partial_{t}\left(\frac{1}{\sqrt{g}}\right) \operatorname{det} A^{1}-\int_{\Gamma_{1}} \frac{1}{\sqrt{g}} \operatorname{det} A^{2}-\int_{\Gamma_{1}} \frac{1}{\sqrt{g}} \operatorname{det} A^{3} \\
& =K_{211}+K_{212}+K_{213}+K_{214} .
\end{aligned}
$$

Note that $\left\|\partial_{t}\left(\sqrt{g}^{-1}\right)\right\|_{L^{\infty}\left(\Gamma_{1}\right)} \lesssim P\left(\|\eta\|_{H^{2.5+\delta_{0}}}\right)\|v\|_{H^{2.5+\delta_{0}}}$ Since also $\left|\operatorname{det} A^{1}\right| \lesssim|\bar{\partial} \eta|^{2}(\mathcal{E} \bar{\partial} v)^{2}$, we get

$$
K_{212} \lesssim P\left(\|\eta\|_{H^{2.5+\delta_{0}}}\right)\|v\|_{H^{2.5+\delta_{0}}}\|\mathcal{E} \bar{\partial} v\|_{L^{2}\left(\Gamma_{1}\right)}^{2} \lesssim P\left(\|\eta\|_{H^{2.5+\delta_{0}}}\right)\|v\|_{H^{2.5+\delta_{0}}}\|\mathcal{E} v\|_{H^{1.5}}^{2} .
$$

Similarly,

$$
\left|\int_{\Gamma_{1}} \frac{1}{\sqrt{g}}\left(\operatorname{det} A^{2}+\operatorname{det} A^{3}\right)\right| \lesssim P\left(\|\eta\|_{H^{2.5+\delta_{0}}}\right)\|\mathcal{E} \bar{\partial} v\|_{L^{2}\left(\Gamma_{1}\right)}^{2} \lesssim P\left(\|\eta\|_{H^{2.5+\delta_{0}}}\right)\|\mathcal{E} v\|_{H^{1.5}}^{2} .
$$


The term $K_{211}$ requires more care since if we bound $\operatorname{det} A^{1}$ as above, we obtain the term $\|\mathcal{E} \bar{\partial} v\|_{L^{2}\left(\Gamma_{1}\right)}^{2}$ which cannot be absorbed into the left side. Instead we integrate by parts and obtain

$$
\begin{aligned}
\int_{\Gamma_{1}} \frac{1}{\sqrt{g}} \operatorname{det} A^{1}= & \int_{\Gamma_{1}} \frac{1}{\sqrt{g}}\left(\partial_{1} \eta_{\mu} \partial_{2} \eta_{\lambda} \partial_{1} \mathcal{E} v^{\mu} \partial_{2} \mathcal{E} v^{\lambda}-\partial_{1} \eta_{\mu} \partial_{2} \eta_{\lambda} \partial_{2} \mathcal{E} v^{\mu} \partial_{1} \mathcal{E} v^{\lambda}\right) \\
= & \int_{\Gamma_{1}} \frac{1}{\sqrt{g}}\left(-\partial_{1} \eta_{\mu} \partial_{2} \eta_{\lambda} \mathcal{E} v^{\mu} \partial_{1} \partial_{2} \mathcal{E} v^{\lambda}+\partial_{1} \eta_{\mu} \partial_{2} \eta_{\lambda} \mathcal{E} v^{\mu} \partial_{2} \partial_{1} \mathcal{E} v^{\lambda}\right) \\
& -\int_{\Gamma_{1}} Q_{\mu \lambda}^{i}\left(\bar{\partial} \eta, \bar{\partial}^{2} \eta\right) \mathcal{E} v^{\mu} \partial_{i} \mathcal{E} v^{\lambda} \\
= & -\int_{\Gamma_{1}} Q_{\mu \lambda}^{i}\left(\bar{\partial} \eta, \bar{\partial}^{2} \eta\right) \mathcal{E} v^{\mu} \partial_{i} \mathcal{E} v^{\lambda},
\end{aligned}
$$

where $Q_{\mu \lambda}^{i}\left(\bar{\partial} \eta, \bar{\partial}^{2} \eta\right)$ is a rational function, which is linear in $\bar{\partial}^{2} \eta$ and can thus be written as $Q_{\mu \lambda}^{i}\left(\bar{\partial} \eta, \bar{\partial}^{2} \eta\right)=$ $\tilde{Q}_{\mu \lambda}^{i}(\bar{\partial} \eta) \bar{\partial}^{2} \eta$ with $\tilde{Q}$ a rational function. Hence, $K_{211}=(d / d t) \int_{\Gamma_{1}} \tilde{Q}_{\mu \lambda}^{i}(\bar{\partial} \eta) \bar{\partial}^{2} \eta \mathcal{E} v^{\mu} \partial_{i} \mathcal{E} v^{\lambda}$, and thus

$$
K_{21} \lesssim \frac{d}{d t} \int_{\Gamma_{1}} \tilde{Q}_{\mu \lambda}^{i}(\bar{\partial} \eta) \bar{\partial}^{2} \eta \mathcal{E} v^{\mu} \partial_{i} \mathcal{E} v^{\lambda}+P\left(\|\eta\|_{H^{2.5+\delta_{0}}}\right)\left(\|v\|_{H^{2.5+\delta_{0}}}+1\right)\|\mathcal{E} v\|_{H^{1.5}}^{2} .
$$

We summarize the above derivations in the following statement.

Lemma 6.1. Consider the integral $K=-\int_{\Gamma_{1}} \mathcal{E} \partial_{t}\left(a^{\mu \alpha} q\right) \mathcal{E} \partial_{t} v_{\alpha} N_{\mu}$, where $\mathcal{E}$ is a differential operator which commutes with $\partial_{t}$ and $\partial_{\alpha}$, i.e., (5.6) and (5.7) hold. Then we have

$$
\begin{aligned}
-\int_{\Gamma_{1}} \mathcal{E} \partial_{t}\left(a^{\mu \alpha} q\right) \mathcal{E} \partial_{t} v_{\alpha} N_{\mu} \\
\lesssim-\frac{1}{2} \frac{d}{d t} \int_{\Gamma_{1}} \sqrt{g} g^{i j} \Pi_{\lambda}^{\mu} \mathcal{E} \partial_{j} v^{\lambda} \Pi_{\mu}^{\alpha} \mathcal{E} \partial_{i} v_{\alpha}+\frac{d}{d t} \int_{\Gamma_{1}} \tilde{Q}_{\mu \lambda}^{i}(\bar{\partial} \eta) \bar{\partial}^{2} \eta \mathcal{E} v^{\mu} \partial_{i} \mathcal{E} v^{\lambda} \\
-\int_{\Gamma_{1}}\left(\mathcal{E}\left(\sqrt{g} g^{i j} \Pi_{\lambda}^{\alpha} \partial_{j} v^{\lambda}\right)-\sqrt{g} g^{i j} \Pi_{\lambda}^{\alpha} \mathcal{E} \partial_{j} v^{\lambda}\right) \partial_{i} \mathcal{E} \partial_{t} v_{\alpha} \\
-\int_{\Gamma_{1}}\left(\mathcal{E}\left(\sqrt{g}\left(g^{i j} g^{k l}-g^{l j} g^{i k}\right) \partial_{j} \eta^{\alpha} \partial_{k} \eta_{\lambda} \partial_{l} v^{\lambda}\right)\right. \\
\left.-\sqrt{g}\left(g^{i j} g^{k l}-g^{l j} g^{i k}\right) \partial_{j} \eta^{\alpha} \partial_{k} \eta_{\lambda} \partial_{l} \mathcal{E} v^{\lambda}\right) \partial_{i} \mathcal{E} \partial_{t} v_{\alpha} \\
+P\left(\|\eta\|_{H^{2.5+\delta_{0}}}\right)\left(\|v\|_{H^{2.5+\delta_{0}}}+1\right)\|\mathcal{E} v\|_{H^{1.5}}^{2}
\end{aligned}
$$

Note that the third and the fourth terms are of commutator type. Since it is needed in the next two sections, we show here an estimate for the time integral of the second term on the right side of (6.4). We have

$$
\begin{aligned}
\int_{\Gamma_{1}} & \left.\tilde{Q}_{\mu \lambda}^{i}(\bar{\partial} \eta) \bar{\partial}^{2} \eta \mathcal{E} v^{\mu} \partial_{i} \mathcal{E} v^{\lambda}\right|_{t} \lesssim\left\|\tilde{Q}_{\mu \lambda}^{i}(\bar{\partial} \eta) \bar{\partial}^{2} \eta\right\|_{H^{0.5-\nu}\left(\Gamma_{1}\right)}\left\|\mathcal{E} v^{\mu}\right\|_{H^{0.5+\nu}\left(\Gamma_{1}\right)}\left\|\partial_{i} \mathcal{E} v^{\lambda}\right\|_{L^{2}\left(\Gamma_{1}\right)} \\
& \lesssim\left\|\tilde{Q}(\bar{\partial} \eta) \bar{\partial}^{2} \eta\right\|_{H^{0.5-\nu}\left(\Gamma_{1}\right)}\|\mathcal{E} v\|_{H^{0.5+\nu}\left(\Gamma_{1}\right)}\|\bar{\partial} \mathcal{E} v\|_{L^{2}\left(\Gamma_{1}\right)} \\
& \lesssim\left(\|\tilde{Q}(\bar{\partial} \eta)\|_{L^{\infty}}+\|\tilde{Q}(\bar{\partial} \eta)\|_{H^{1}\left(\Gamma_{1}\right)}\right)\left\|\bar{\partial}^{2} \eta\right\|_{H^{0.5-\nu}\left(\Gamma_{1}\right)}\|\mathcal{E} v\|_{H^{0.5+\nu}\left(\Gamma_{1}\right)}\left\|\partial_{i} \mathcal{E} v\right\|_{L^{2}\left(\Gamma_{1}\right)},
\end{aligned}
$$

where we used

$$
\|A B\|_{H^{0.5-\nu}\left(\Gamma_{1}\right)} \lesssim\left(\|A\|_{L^{\infty}\left(\Gamma_{1}\right)}+\|A\|_{H^{1}\left(\Gamma_{1}\right)}\right)\|B\|_{H^{0.5-\nu}\left(\Gamma_{1}\right)}
$$

in the last inequality. Note that (6.6) follows by a simple application of the Kato-Ponce fractional chain rule. Using that $H^{1+\delta_{0}}\left(\Gamma_{1}\right)$ is an algebra, we obtain from (6.5)

$$
\begin{aligned}
\left.\int_{\Gamma_{1}} \tilde{Q}_{\mu \lambda}^{i}(\bar{\partial} \eta) \bar{\partial}^{2} \eta \mathcal{E} v^{\mu} \partial_{i} \mathcal{E} v^{\lambda}\right|_{t} \lesssim P\left(\|\bar{\partial} \eta\|_{H^{1+\delta_{0}}\left(\Gamma_{1}\right)}\right)\left\|\bar{\partial}^{2} \eta\right\|_{H^{0.5-\nu}\left(\Gamma_{1}\right)}\|\mathcal{E} v\|_{H^{1+\nu}}\|\mathcal{E} v\|_{H^{1.5}} \\
\quad \lesssim P\left(\|\eta\|_{H^{2.5+\delta_{0}}}\right)\|\eta\|_{H^{3-\nu}}\|\mathcal{E} v\|_{L^{2}}^{(1-2 \nu) / 3}\|\mathcal{E} v\|_{H^{1.5}}^{(5+2 \nu) / 3} \\
\quad \lesssim P\left(\|\eta\|_{H^{3-\nu}}\right)\left(\|\mathcal{E} v(0)\|_{L^{2}}^{2}+\int_{0}^{t}\left\|\mathcal{E} \partial_{t} v\right\|_{L^{2}}^{2}\right)+\epsilon_{0}\|\mathcal{E} v\|_{H^{1.5}}^{2}
\end{aligned}
$$


from where, by Lemma 3.1(i),

$$
\int_{\Gamma_{1}} \tilde{Q}_{\mu \lambda}^{i}(\bar{\partial} \eta) \bar{\partial}^{2} \eta \mathcal{E} v \partial_{i} \mathcal{E} v \lesssim\|\mathcal{E} v(0)\|_{L^{2}}^{2}+\int_{0}^{t}\left\|\mathcal{E} \partial_{t} v\right\|_{L^{2}}^{2}+\epsilon_{0}\|\mathcal{E} v\|_{H^{1.5}}^{2}
$$

\section{The tangential estimate ON $\partial_{t} v$}

In this and the next sections, we perform energy estimates on the quantity $\left\|\mathcal{E} \partial_{t} v\right\|_{L^{2}}$ with $\mathcal{E}=\tilde{\partial}^{1-\nu / 2}$ and $\mathcal{E}=\partial_{t}$, respectively, where $\tilde{\partial}=\left(I-\Delta_{2}\right)^{1 / 2}$ with $\Delta_{2}=\partial_{1}^{2}+\partial_{2}^{2}$ denoting the horizontal Laplacian. In both cases, we apply $\mathcal{E} \partial_{t}$ to (2.2), multiply the resulting equation with $\mathcal{E} \partial_{t} v$, and integrate, obtaining

$$
\frac{1}{2} \frac{d}{d t}\left\|\mathcal{E} \partial_{t} v\right\|_{L^{2}}^{2}=-\int_{\Omega} \mathcal{E} \partial_{t}\left(a^{\mu \alpha} \partial_{\mu} q\right) \mathcal{E} \partial_{t} v_{\alpha}=\int_{\Omega} \mathcal{E} \partial_{t}\left(a^{\mu \alpha} q\right) \mathcal{E} \partial_{t} \partial_{\mu} v_{\alpha}-\int_{\Gamma_{1}} \mathcal{E} \partial_{t}\left(a^{\mu \alpha} q\right) \mathcal{E} \partial_{t} v_{\alpha} N_{\mu}
$$

since $-\int_{\Gamma_{0}} \mathcal{E} \partial_{t}\left(a^{\mu \alpha} q\right) \mathcal{E} \partial_{t} v_{\alpha} N_{\mu}=0$ by (2.7) and $a^{31}=a^{32}=0$ on $\Gamma_{0}$ due to $a^{31}=\partial_{1} \eta^{2} \partial_{2} \eta^{3}-\partial_{2} \eta^{2} \partial_{1} \eta^{3}$ and $a^{32}=\partial_{2} \eta^{1} \partial_{1} \eta^{3}-\partial_{1} \eta^{1} \partial_{2} \eta^{3}$.

In this section, we set $\mathcal{E}=\tilde{\partial}^{1-\nu / 2}$. The most important assertion in the next statement is that it provides control of $\left\|v^{3}\right\|_{H^{2-\nu / 2}\left(\Gamma_{1}\right)}$ needed further below.

Lemma 7.1. The Lagrangian velocity $v$ and its derivative $\partial_{t} v$ satisfy

$$
\left\|\tilde{\partial}^{1-\nu / 2} \partial_{t} v\right\|_{L^{2}}^{2}+\left\|\Pi \bar{\partial} \tilde{\partial}^{1-\nu / 2} v\right\|_{L^{2}\left(\Gamma_{1}\right)}^{2} \lesssim P_{0}+\epsilon_{0}\|v\|_{H^{2.5-\nu / 2}}^{2}+\int_{0}^{t} P,
$$

where $P$ is a polynomial in $\|v\|_{H^{3-\nu}},\left\|\partial_{t} v\right\|_{H^{1.5}},\|q\|_{H^{2.5-\nu / 2}}$, and $\left\|\partial_{t} q\right\|_{H^{1}}$, while $P_{0}$ is a polynomial in $\left\|v_{0}\right\|_{H^{3-\nu}}$ and $\left\|\partial_{t} v(0)\right\|_{H^{1.5}}$.

Using the notation (2.12)-(2.14), the inequality (7.2) implies

$$
\left\|\Pi \bar{\partial} \tilde{\partial}^{1-\nu / 2} v\right\|_{L^{2}\left(\Gamma_{1}\right)}^{2} \lesssim \epsilon_{0} E_{0}^{2}+P_{0}+\int_{0}^{t} P
$$

where, as mentioned above, $\epsilon_{0}>0$ denotes an arbitrarily small constant.

Proof of Lemma 7.1. From (7.1), we have the equation

$$
\frac{1}{2} \frac{d}{d t}\left\|\tilde{\partial}^{1-\nu / 2} \partial_{t} v\right\|_{L^{2}}^{2}=I_{1}+I_{2}+I_{3}+I_{4},
$$

where

$$
\begin{array}{ll}
I_{1}=\int_{\Omega} \tilde{\partial}^{1-\nu / 2}\left(\partial_{t} a^{\mu \alpha} q\right) \tilde{\partial}^{1-\nu / 2} \partial_{t} \partial_{\mu} v_{\alpha}, & I_{2}=\int_{\Omega} a^{\mu \alpha} \tilde{\partial}^{2-\nu} \partial_{t} q \partial_{t} \partial_{\mu} v_{\alpha} \\
I_{3}=\int_{\Omega}\left(\tilde{\partial}^{2-\nu}\left(a^{\mu \alpha} \partial_{t} q\right)-a^{\mu \alpha} \tilde{\partial}^{2-\nu} \partial_{t} q\right) \partial_{t} \partial_{\mu} v_{\alpha}, & I_{4}=-\int_{\Gamma_{1}} \tilde{\partial}^{1-\nu / 2} \partial_{t}\left(a^{\mu \alpha} q\right) \tilde{\partial}^{1-\nu / 2} \partial_{t} v_{\alpha} N_{\mu} .
\end{array}
$$

Using multiplicative Sobolev inequalities, we have

$$
\begin{aligned}
I_{1} & =\int_{\Omega} \tilde{\partial}^{1.5-\nu}\left(\partial_{t} a^{\mu \alpha} q\right) \tilde{\partial}^{0.5} \partial_{t} \partial_{\mu} v_{\alpha} \lesssim \sum_{\mu, \alpha}\left\|\tilde{\partial}^{1.5-\nu}\left(\partial_{t} a^{\mu \alpha} q\right)\right\|_{L^{2}}\left\|\partial_{t} v\right\|_{H^{1.5}} \\
& \lesssim \sum_{\mu, \alpha}\left\|\partial_{t} a^{\mu \alpha} q\right\|_{H^{1.5-\nu}}\left\|\partial_{t} v\right\|_{H^{1.5}} \lesssim\left\|\partial_{t} a\right\|_{H^{2-\nu}}\|q\|_{H^{1}}\left\|\partial_{t} v\right\|_{H^{1.5}}+\left\|\partial_{t} a\right\|_{H^{1}}\|q\|_{H^{2-\nu}}\left\|\partial_{t} v\right\|_{H^{1.5}} \lesssim P
\end{aligned}
$$

using $L^{2}$ based Kato-Ponce type estimates (fractional product rule), as in [56, 57]. For the second term in (7.4), we use the divergence-free condition (2.3) to write

$$
\begin{aligned}
I_{2} & =-\int_{\Omega} \partial_{t} a^{\mu \alpha} \tilde{\partial}^{2-\nu} \partial_{t} q \partial_{\mu} v_{\alpha}=-\int_{\Omega} \partial_{t} a^{\mu \alpha} \partial_{\mu} v_{\alpha} \tilde{\partial}^{1-\nu} \tilde{\partial} \partial_{t} q=-\int \tilde{\partial}^{1-\nu}\left(\partial_{t} a^{\mu \alpha} \partial_{\mu} v_{\alpha}\right) \tilde{\partial} \partial_{t} q \\
& \lesssim\left\|\tilde{\partial}^{1-\nu}\left(\partial_{t} a^{\mu \alpha} \partial_{\mu} v_{\alpha}\right)\right\|_{L^{2}}\left\|\partial_{t} q\right\|_{H^{1}} \\
& \lesssim\left\|\tilde{\partial}^{1-\nu} \partial_{t} a^{\mu \alpha}\right\|_{H^{1}}\left\|\partial_{\mu} v_{\alpha}\right\|_{H^{0.5}}\left\|\partial_{t} q\right\|_{H^{1}}+\left\|\partial_{t} a^{\mu \alpha}\right\|_{H^{1}}\left\|\tilde{\partial}^{1-\nu} \partial_{\mu} v_{\alpha}\right\|_{H^{0.5}}\left\|\partial_{t} q\right\|_{H^{1}} \lesssim P,
\end{aligned}
$$


again using the fractional chain rule. The last interior term $I_{3}$ is estimated as

$$
I_{3} \lesssim\left\|\tilde{\partial}^{2-\nu}\left(a^{\mu \alpha} \partial_{t} q\right)-a^{\mu \alpha} \tilde{\partial}^{2-\nu} \partial_{t} q\right\|_{L^{3 / 2}}\left\|\partial_{t} \partial_{\mu} v\right\|_{L^{3}} \lesssim\|a\|_{H^{2-\nu}}\left\|\partial_{t} q\right\|_{H^{1}}\left\|\partial_{t} v\right\|_{H^{1.5}} \lesssim P
$$

For completeness, we show the validity of the second inequality above as the Kato-Ponce inequality can only be applied in the first two variables. We do so by the successive integration. For any fixed $x_{3} \in(0,1)$, we employ the Kato-Ponce inequality to obtain

$$
\left\|\tilde{\partial}^{2-\nu}\left(a^{\mu \alpha} \partial_{t} q\right)-a^{\mu \alpha} \tilde{\partial}^{2-\nu} \partial_{t} q\right\|_{L_{x_{1}, x_{2}}^{3 / 2}} \lesssim\left\|\tilde{\partial}^{2-\nu} a\right\|_{L_{x_{1}, x_{2}}^{2}}\left\|\partial_{t} q\right\|_{L_{x_{1}, x_{2}}^{6}}+\|\tilde{\partial} a\|_{L_{x_{1}, x_{2}}^{6 /(1+2 \nu)}}\left\|\tilde{\partial}^{1-\nu} \partial_{t} q\right\|_{L_{x_{1}, x_{2}}^{6 /(3-2 \nu)}}
$$

(cf. [53, 42, 55, 59]), where $L_{x_{1}, x_{2}}^{p}$ denotes the $L^{p}$ norm in $\left(x_{1}, x_{2}\right)$. Taking the $L_{x_{3}}^{3 / 2}$ norm of both sides and applying the Hölder inequality in the $x_{3}$ variable gives

$$
\begin{aligned}
& \left\|\tilde{\partial}^{2-\nu}\left(a^{\mu \alpha} \partial_{t} q\right)-a^{\mu \alpha} \tilde{\partial}^{2-\nu} \partial_{t} q\right\|_{L^{3 / 2}} \\
& \quad \lesssim\|\| \tilde{\partial}^{2-\nu} a\left\|_{L_{x_{1}, x_{2}}^{2}}\right\| \partial_{t} q\left\|_{L_{x_{1}, x_{2}}^{6}}\right\|_{L_{x_{3}}^{3 / 2}}+\|\| \tilde{\partial} a\left\|_{L_{x_{1}, x_{2}}^{6 /(1+2 \nu)}}\right\| \tilde{\partial}^{1-\nu} \partial_{t} q\left\|_{L_{x_{1}, x_{2}}^{6 /(3-2 \nu)}}\right\|_{L_{x_{3}}^{3 / 2}} \\
& \quad \lesssim\left\|\tilde{\partial}^{2-\nu} a\right\|_{L^{2}}\left\|\partial_{t} q\right\|_{L^{6}}+\|\tilde{\partial} a\|_{L^{6 /(1+2 \nu)}}\left\|\tilde{\partial}^{1-\nu} \partial_{t} q\right\|_{L^{6 /(3-2 \nu)}} \\
& \quad \lesssim\left\|\tilde{\partial}^{2-\nu} a\right\|_{L^{2}}\left\|\partial_{t} q\right\|_{L^{6}}+\|a\|_{H^{2-\nu}}\left\|\partial_{t} q\right\|_{H^{1}}
\end{aligned}
$$

where we used the Sobolev inequality in the last step.

Finally, we use Lemma 6.1 with $\mathcal{E}=\tilde{\partial}^{1-\nu / 2}$ to write

$$
\begin{aligned}
I_{4} \lesssim-\frac{1}{2} \frac{d}{d t} & \int_{\Gamma_{1}} \sqrt{g} g^{i j} \Pi_{\lambda}^{\mu} \tilde{\partial}^{1-\nu / 2} \partial_{j} v^{\lambda} \Pi_{\mu}^{\alpha} \tilde{\partial}^{1-\nu / 2} \partial_{i} v_{\alpha}+\frac{d}{d t} \int_{\Gamma_{1}} \tilde{Q}_{\mu \lambda}^{i}(\bar{\partial} \eta) \bar{\partial}^{2} \eta \tilde{\partial}^{1-\nu / 2} v^{\mu} \partial_{i} \tilde{\partial}^{1-\nu / 2} v^{\lambda} \\
& -\int_{\Gamma_{1}}\left(\tilde{\partial}^{1-\nu / 2}\left(\sqrt{g} g^{i j} \Pi_{\lambda}^{\alpha} \partial_{j} v^{\lambda}\right)-\sqrt{g} g^{i j} \Pi_{\lambda}^{\alpha} \tilde{\partial}^{1-\nu / 2} \partial_{j} v^{\lambda}\right) \partial_{i} \tilde{\partial}^{1-\nu / 2} \partial_{t} v_{\alpha} \\
& -\int_{\Gamma_{1}}\left(\tilde{\partial}^{1-\nu / 2}\left(\sqrt{g}\left(g^{i j} g^{k l}-g^{l j} g^{i k}\right) \partial_{j} \eta^{\alpha} \partial_{k} \eta_{\lambda} \partial_{l} v^{\lambda}\right)\right. \\
& \left.-\sqrt{g}\left(g^{i j} g^{k l}-g^{l j} g^{i k}\right) \partial_{j} \eta^{\alpha} \partial_{k} \eta_{\lambda} \partial_{l} \tilde{\partial}^{1-\nu / 2} v^{\lambda}\right) \partial_{i} \tilde{\partial}^{1-\nu / 2} \partial_{t} v_{\alpha} \\
& +P\left(\|\eta\|_{H^{2.5+\delta_{0}}}\right)\left(\|v\|_{H^{2.5+\delta_{0}}}+1\right)\|v\|_{H^{2.5-\nu / 2}}^{2}
\end{aligned}
$$

where, recall, $\delta_{0}>0$ is arbitrarily small. The first term in (7.6) leads to the second term of (7.2). Namely, using

$$
\sqrt{g} g^{i j} \xi_{i} \xi_{j} \geq \frac{1}{C}|\xi|^{2}, \quad \xi \in \mathbb{R}^{2}
$$

for $t$ as in Lemma 3.1(iv), we get

$$
\frac{1}{2} \int_{\Gamma_{1}} \sqrt{g} g^{i j} \Pi_{\lambda}^{\mu} \tilde{\partial}^{1-\nu / 2} \partial_{j} v^{\lambda} \Pi_{\mu}^{\alpha} \tilde{\partial}^{1-\nu / 2} \partial_{i} v_{\alpha} \geq \frac{1}{C} \int_{\Gamma_{1}} \Pi_{\lambda}^{\mu} \tilde{\partial}^{1-\nu / 2} \partial_{i} v^{\lambda} \Pi_{\mu}^{\alpha} \tilde{\partial}^{1-\nu / 2} \partial_{i} v_{\alpha}=\frac{1}{C}\left\|\Pi \bar{\partial}\left(\tilde{\partial}^{1-\nu / 2} v\right)\right\|_{L^{2}\left(\Gamma_{1}\right)}^{2} .
$$

In order to establish (7.7), we write $g^{i j} \xi_{i} \xi_{j}=|\xi|^{2}+\left(\sqrt{g} g^{i j}-\delta^{i j}\right) \xi_{i} \xi_{j}$ and appeal to Lemma 3.1(iv).

Note that the last term in (7.6) is dominated by $P$. We integrate the inequality (7.6) in time on $[0, t]$ and then integrate by parts in time in the third and the fourth terms. Since both integrals are treated the same way, we only estimate the time integral of the third term. Denoting

$$
A^{i \alpha}=\tilde{\partial}^{1-\nu / 2}\left(\sqrt{g} g^{i j} \Pi_{\lambda}^{\alpha} \partial_{j} v^{\lambda}\right)-\sqrt{g} g^{i j} \Pi_{\lambda}^{\alpha} \tilde{\partial}^{1-\nu / 2} \partial_{j} v^{\lambda}
$$


we have

$$
\begin{aligned}
& \left\|A^{i \alpha}\right\|_{L^{2}\left(\Gamma_{1}\right)} \lesssim\left\|\tilde{\partial}^{1-\nu / 2}\left(\sqrt{g} g^{i j} \Pi_{\lambda}^{\alpha}\right)\right\|_{L^{4 /(1+\nu)}\left(\Gamma_{1}\right)}\left\|\partial_{j} v^{\lambda}\right\|_{L^{4 /(1-\nu)}\left(\Gamma_{1}\right)} \\
& +\left\|\tilde{\partial}\left(\sqrt{g} g^{i j} \Pi_{\lambda}^{\alpha}\right)\right\|_{L^{4 /(1+2 \nu)}\left(\Gamma_{1}\right)}\left\|\tilde{\partial}^{-\nu / 2} \partial_{j} v^{\lambda}\right\|_{L^{4 /(1-2 \nu)}\left(\Gamma_{1}\right)} \\
& \lesssim\left\|\tilde{\partial}^{1-\nu / 2}\left(\sqrt{g} g^{i j} \Pi_{\lambda}^{\alpha}\right)\right\|_{H^{(1-\nu) / 2}\left(\Gamma_{1}\right)}\left\|\partial_{j} v^{\lambda}\right\|_{H^{(1+\nu) / 2}\left(\Gamma_{1}\right)} \\
& +\left\|\tilde{\partial}\left(\sqrt{g} g^{i j} \Pi_{\lambda}^{\alpha}\right)\right\|_{H^{1 / 2-\nu}\left(\Gamma_{1}\right)}\left\|\tilde{\partial}^{-\nu / 2} \partial_{j} v^{\lambda}\right\|_{H^{1 / 2+\nu}\left(\Gamma_{1}\right)} \\
& \lesssim\left\|\tilde{\partial}^{1-\nu / 2}\left(\sqrt{g} g^{i j} \Pi_{\lambda}^{\alpha}\right)\right\|_{H^{1-\nu / 2}}\left\|\partial_{j} v^{\lambda}\right\|_{H^{1+\nu / 2}} \\
& +\left\|\tilde{\partial}\left(\sqrt{g} g^{i j} \Pi_{\lambda}^{\alpha}\right)\right\|_{H^{1-\nu}}\left\|\tilde{\partial}^{-\nu / 2} \partial_{j} v^{\lambda}\right\|_{H^{1+\nu}} \lesssim P\left(\|\eta\|_{H^{3-\nu}}\right)\|v\|_{H^{2+\nu / 2}}
\end{aligned}
$$

where we used the commutator inequality (2.11) in [54]. Now, the time integral of the third term on the right side of (7.6) may then be estimated using integration by parts in time as

$$
\begin{aligned}
&-\int_{0}^{t} \int_{\Gamma_{1}}\left(\tilde{\partial}^{1-\nu / 2}\left(\sqrt{g} g^{i j} \Pi_{\lambda}^{\alpha} \partial_{j} v^{\lambda}\right)-\sqrt{g} g^{i j} \Pi_{\lambda}^{\alpha} \tilde{\partial}^{1-\nu / 2} \partial_{j} v^{\lambda}\right) \partial_{i} \tilde{\partial}^{1-\nu / 2} \partial_{t} v_{\alpha} \\
&=- \int_{0}^{t} \int_{\Gamma_{1}} A^{i \alpha} \partial_{i} \tilde{\partial}^{1-\nu / 2} \partial_{t} v_{\alpha}=-\left.\int_{\Gamma_{1}} A^{i \alpha} \partial_{i} \tilde{\partial}^{1-\nu / 2} v_{\alpha}\right|_{0} ^{t}+\int_{0}^{t} \int_{\Gamma_{1}} \partial_{t} A^{i \alpha} \partial_{i} \tilde{\partial}^{1-\nu / 2} v_{\alpha} \\
&=-\left.\int_{\Gamma_{1}} A^{i \alpha} \partial_{i} \tilde{\partial}^{1-\nu / 2} v_{\alpha}\right|_{0} ^{t} \\
&+\int_{0}^{t} \int_{\Gamma_{1}}\left(\tilde{\partial}^{1-\nu / 2}\left(\sqrt{g} g^{i j} \Pi_{\lambda}^{\alpha} \partial_{j} \partial_{t} v^{\lambda}\right)-\sqrt{g} g^{i j} \Pi_{\lambda}^{\alpha} \tilde{\partial}^{1-\nu / 2} \partial_{j} \partial_{t} v^{\lambda}\right) \partial_{i} \tilde{\partial}^{1-\nu / 2} v_{\alpha} \\
&+\int_{0}^{t} \int_{\Gamma_{1}}\left(\tilde{\partial}^{1-\nu / 2}\left(\partial_{t}\left(\sqrt{g} g^{i j} \Pi_{\lambda}^{\alpha}\right) \partial_{j} v^{\lambda}\right)-\partial_{t}\left(\sqrt{g} g^{i j} \Pi_{\lambda}^{\alpha}\right) \tilde{\partial}^{1-\nu / 2} \partial_{j} v^{\lambda}\right) \partial_{i} \tilde{\partial}^{1-\nu / 2} v_{\alpha} \\
& \lesssim P_{0}+P\left(\|\eta\|_{H^{3-\nu}}\right)\|v\|_{H^{2+\nu / 2}}\|v\|_{H^{2.5-\nu / 2}}+\int_{0}^{t} P \\
& \lesssim P_{0}+\epsilon_{0}\|v\|_{H^{2.5-\nu / 2}}^{2}+\int_{0}^{t} P .
\end{aligned}
$$

We estimate the fourth term in (7.6) the same way. For the second term on the right side of (7.6), we use (6.7) and obtain

$$
\int_{\Gamma_{1}} \tilde{Q}_{\mu \lambda}^{i}(\bar{\partial} \eta) \bar{\partial}^{2} \eta \tilde{\partial}^{1-\nu / 2} v^{\mu} \partial_{i} \tilde{\partial}^{1-\nu / 2} v^{\lambda} \lesssim P_{0}+\int_{0}^{t} P+\epsilon_{0}\|v\|_{H^{2.5-\nu / 2}}^{2}
$$

Collecting all the estimates and using the bound (7.8), we obtain $\int_{0}^{t} I_{4} \lesssim P_{0}+\epsilon_{0}\|v\|_{H^{2.5-\nu / 2}}^{2}+\int_{0}^{t} P$, and (7.2) follows.

\section{The $L^{2}$ ESTIMATE ON $\partial_{t}^{2} v$}

We have (7.1) with $\mathcal{E}=\partial_{t}$, i.e.,

We rewrite this as

$$
\frac{1}{2} \frac{d}{d t}\left\|\partial_{t}^{2} v\right\|_{L^{2}}^{2}=\int_{\Omega} \partial_{t}^{2}\left(a^{\mu \alpha} q\right) \partial_{t}^{2} \partial_{\mu} v_{\alpha}-\int_{\Gamma_{1}} \partial_{t}^{2}\left(a^{\mu \alpha} q\right) \partial_{t}^{2} v_{\alpha} N_{\mu} .
$$

where

$$
\frac{1}{2} \frac{d}{d t}\left\|\partial_{t}^{2} v\right\|_{L^{2}}^{2}=J_{1}+J_{2}+J_{3}+J_{4}
$$

$$
\begin{array}{ll}
J_{1}=\int_{\Omega} \partial_{t}^{2} a^{\mu \alpha} q \partial_{t}^{2} \partial_{\mu} v_{\alpha}, & J_{2}=2 \int_{\Omega} \partial_{t} a^{\mu \alpha} \partial_{t} q \partial_{t}^{2} \partial_{\mu} v_{\alpha} \\
J_{3}=\int_{\Omega} a^{\mu \alpha} \partial_{t}^{2} q \partial_{t}^{2} \partial_{\mu} v_{\alpha}, & J_{4}=-\int_{\Gamma_{1}} \partial_{t}^{2}\left(a^{\mu \alpha} q\right) \partial_{t}^{2} v_{\alpha} N_{\mu}
\end{array}
$$


Lemma 8.1. The time derivative of the Lagrangian velocity $\partial_{t} v$ and its second derivative $\partial_{t}^{2} v$ satisfy

$$
\begin{aligned}
\left\|\partial_{t}^{2} v\right\|_{L^{2}}^{2}+ & \left\|\Pi \bar{\partial} \partial_{t} v\right\|_{L^{2}\left(\Gamma_{1}\right)}^{2} \\
\lesssim & \|q\|_{H^{1}}\|v\|_{H^{1.5}}^{(3-2 \nu) /(2-\nu)}\|v\|_{H^{2.5-\nu / 2}}^{1 /(2-\nu)}\left\|\partial_{t} v\right\|_{H^{1.5}} \\
& +\|q\|_{H^{1}}^{\left(2-\nu-2 \delta_{0}\right) /(3-\nu)}\|q\|_{H^{2.5-\nu / 2}}^{\left(1+2 \delta_{0}\right) /(3-\nu)}\left\|\partial_{t} v\right\|_{L^{2}}^{2 / 3}\left\|\partial_{t} v\right\|_{H^{1.5}}^{4 / 3}\left(1+\int_{0}^{t} P\right) \\
& +\|v\|_{H^{1.5}}^{(1-\nu) /(2-\nu)}\|v\|_{H^{2.5-\nu / 2}}^{1 /(2-\nu)}\left\|\partial_{t} q\right\|_{H^{1}}\left\|\partial_{t} v\right\|_{L^{2}}^{\left(2-2 \delta_{0}\right) / 3}\left\|\partial_{t} v\right\|_{H^{1.5}}^{\left(1+2 \delta_{0}\right) / 3} \\
& +\left\|\partial_{t} v\right\|_{L^{2}}^{1-\nu / 3}\left\|\partial_{t} v\right\|_{H^{1.5}}^{\nu / 3}\left\|\partial_{t} q\right\|_{H^{1}}\|v\|_{H^{2.5-\nu / 2}} \\
& +\|v\|_{H^{1.5}}^{(1-\nu) /(2-\nu)}\|v\|_{H^{2.5-\nu / 2} /(2-\nu)}^{1 / 2-\nu}\|v\|_{H^{1.5}}^{2}\left\|\partial_{t} q\right\|_{H^{1}} \\
& +\|v\|_{H^{1.5}}^{2(1-\nu) /(2-\nu)}\|v\|_{H^{2.5-\nu / 2}}^{2 /(2-\nu)}\left\|\partial_{t} v\right\|_{H^{1.5}}+\epsilon_{0}\left\|\partial_{t} v\right\|_{H^{1.5}}^{2}+P_{0}+\int_{0}^{t} P
\end{aligned}
$$

where $P$ is a polynomial in $\|v\|_{H^{3-\nu}},\left\|\partial_{t} v\right\|_{H^{1.5}},\left\|\partial_{t}^{2} v\right\|_{L^{2}},\|q\|_{H^{2.5-\nu / 2}}$, and $\left\|\partial_{t} q\right\|_{H^{1}}$ and $P_{0}$ is a polynomial in $\left\|v_{0}\right\|_{H^{3-\nu}},\left\|\partial_{t} v(0)\right\|_{H^{1.5}}$, and $\left\|\partial_{t}^{2} v(0)\right\|_{L^{2}}$.

We recall that $\bar{\partial}$ is given by (6.3). With the notation $G=\|q\|_{H^{2.5-\nu / 2}}$ and $H=\left\|\partial_{t} q\right\|_{H^{1}}$, the equation (8.2) may be rewritten as

$$
\begin{array}{r}
\left\|\partial_{t}^{2} v\right\|_{L^{2}}^{2}+\left\|\Pi \bar{\partial} \partial_{t} v\right\|_{L^{2}\left(\Gamma_{1}\right)}^{2} \\
\lesssim \epsilon_{0} E_{1}^{2}+\left(P_{0}+\int_{0}^{t} P\right)\left(E_{0}^{1 /(2-\nu)} E_{1}+G^{\left(1+2 \delta_{0}\right) /(3-\nu)} E_{1}^{4 / 3}+E_{0}^{1 /(2-\nu)} H E_{1}^{\left(1+2 \delta_{0}\right) / 3}\right. \\
\left.+E_{1}^{\nu / 3} H E_{0}+E_{0}^{1 /(2-\nu)} H+E_{0}^{2 /(2-\nu)} E_{1}+1\right),
\end{array}
$$

from where, taking the square root

$$
\begin{array}{r}
\left\|\partial_{t}^{2} v\right\|_{L^{2}}+\left\|\Pi \bar{\partial} \partial_{t} v\right\|_{L^{2}\left(\Gamma_{1}\right)} \\
\lesssim \epsilon_{0} E_{1}+\left(P_{0}+\int_{0}^{t} P\right)\left(E_{0}^{1 / 2(2-\nu)} E_{1}^{1 / 2}+G^{\left(1+2 \delta_{0}\right) / 2(3-\nu)} E_{1}^{2 / 3}+E_{0}^{1 / 2(2-\nu)} H^{1 / 2} E_{1}^{\left(1+2 \delta_{0}\right) / 6}\right. \\
\left.+E_{1}^{\nu / 6} H^{1 / 2} E_{0}^{1 / 2}+E_{0}^{1 / 2(2-\nu)} H^{1 / 2}+E_{0}^{1 /(2-\nu)} E_{1}^{1 / 2}+1\right),
\end{array}
$$

and then using Young's inequality

$$
\begin{aligned}
& \left\|\partial_{t}^{2} v\right\|_{L^{2}}+\left\|\Pi \bar{\partial} \partial_{t} v\right\|_{L^{2}\left(\Gamma_{1}\right)} \\
& \lesssim \epsilon_{0} E_{1}+\left(P_{0}+\int_{0}^{t} P\right)\left(E_{0}^{1 /(2-\nu)}+G^{3\left(1+2 \delta_{0}\right) / 2(3-\nu)}+E_{0}^{3 /\left(5-2 \delta_{0}\right)(2-\nu)} H^{3 /\left(5-2 \delta_{0}\right)}\right. \\
& \left.+H^{3 /(6-\nu)} E_{0}^{3 /(6-\nu)}+E_{0}^{1 / 2(2-\nu)} H^{1 / 2}+E_{0}^{2 /(2-\nu)}+1\right) .
\end{aligned}
$$

Using the notation (2.14), i.e., $E=E_{0}^{2}+E_{1}+1$, this may be rewritten as

$$
\begin{array}{r}
\left\|\partial_{t}^{2} v\right\|_{L^{2}}+\left\|\Pi \bar{\partial} \partial_{t} v\right\|_{L^{2}\left(\Gamma_{1}\right)} \\
\lesssim \epsilon_{0} E+\left(P_{0}+\int_{0}^{t} P\right)\left(E^{1 / 2(2-\nu)}+G^{3\left(1+2 \delta_{0}\right) / 2(3-\nu)}+E^{3 / 2\left(5-2 \delta_{0}\right)(2-\nu)} H^{3 /\left(5-2 \delta_{0}\right)}\right. \\
\left.+H^{3 /(6-\nu)} E^{3 / 2(6-\nu)}+E^{1 / 4(2-\nu)} H^{1 / 2}+E^{1 /(2-\nu)}+1\right),
\end{array}
$$


where $\delta_{0}>0$ is arbitrarily small. Using Young's inequality on the terms involving $E^{\gamma}$, where $\gamma \in[0,1)$, we get

$$
\begin{array}{r}
\left\|\partial_{t}^{2} v\right\|_{L^{2}}+\left\|\Pi \bar{\partial} \partial_{t} v\right\|_{L^{2}\left(\Gamma_{1}\right)} \\
\lesssim \epsilon_{0} E+\left(P_{0}+\int_{0}^{t} P\right)\left(G^{3\left(1+2 \delta_{0}\right) / 2(3-\nu)}+H^{6(2-\nu) /\left(2\left(5-2 \delta_{0}\right)(2-\nu)-3\right)}\right. \\
\left.+H^{6 /(9-2 \nu)}+H^{2(2-\nu) /(7-4 \nu)}+1\right) .
\end{array}
$$

It is easy to check that the exponents of $H$ are all less than $3 / 4$ for $\delta_{0}>0$ sufficiently small. (In order to verify $6(2-\nu) /\left(2\left(5-2 \delta_{0}\right)(2-\nu)-3\right) \leq 3 / 4$, for $\delta_{0}$ sufficiently small, first set $\delta_{0}=0$ and check that $6(2-\nu) /(10(2-$ $\nu)-3)<3 / 4$ for $\nu \in[0,1 / 2)$.) Therefore,

$$
\left\|\partial_{t}^{2} v\right\|_{L^{2}}+\left\|\Pi \bar{\partial} \partial_{t} v\right\|_{L^{2}\left(\Gamma_{1}\right)} \lesssim \epsilon_{0} E+\left(P_{0}+\int_{0}^{t} P\right)\left(G^{3\left(1+2 \delta_{0}\right) / 2(3-\nu)}+H^{3 / 4}+1\right) .
$$

Proof of Lemma 8.1. Let $J_{1}, J_{2}, J_{3}, J_{4}$ be as in (8.1).

Treatment of $J_{1}$ : For $J_{1}$, we apply Lemma 5.1 with $\mathcal{E}=\partial_{t}$ (that is $\mathcal{D}=\partial_{t}^{2}$ ). We start with the term $L$ in (5.4), which, with $\mathcal{D}=\partial_{t}^{2}$, reads

$$
\begin{aligned}
L & =2 \int_{0}^{t} \int_{\Omega} q \epsilon^{\alpha \lambda \tau} \partial_{2} v_{\lambda} \partial_{3} v_{\tau} \partial_{t}^{2} \partial_{1} v_{\alpha}-2 \int_{0}^{t} \int_{\Omega} q \epsilon^{\alpha \lambda \tau} \partial_{1} v_{\lambda} \partial_{3} v_{\tau} \partial_{t}^{2} \partial_{2} v_{\alpha}+2 \int_{0}^{t} \int_{\Omega} q \epsilon^{\alpha \lambda \tau} \partial_{1} v_{\lambda} \partial_{2} v_{\tau} \partial_{t}^{2} \partial_{3} v_{\alpha} \\
& =L_{1}+L_{2}+L_{3} .
\end{aligned}
$$

We only treat the first term as the other two are handled similarly. Integrating by parts in time, we have

$$
\begin{aligned}
L_{1}= & \left.2 \int_{\Omega} q \epsilon^{\alpha \lambda \tau} \partial_{2} v_{\lambda} \partial_{3} v_{\tau} \partial_{t} \partial_{1} v_{\alpha}\right|_{0} ^{t}-2 \int_{0}^{t} \int_{\Omega} \partial_{t} q \epsilon^{\alpha \lambda \tau} \partial_{2} v_{\lambda} \partial_{3} v_{\tau} \partial_{t} \partial_{1} v_{\alpha} \\
& \quad-2 \int_{0}^{t} \int_{\Omega} q \epsilon^{\alpha \lambda \tau} \partial_{2} \partial_{t} v_{\lambda} \partial_{3} v_{\tau} \partial_{t} \partial_{1} v_{\alpha}-2 \int_{0}^{t} \int_{\Omega} q \epsilon^{\alpha \lambda \tau} \partial_{2} v_{\lambda} \partial_{3} \partial_{t} v_{\tau} \partial_{t} \partial_{1} v_{\alpha} \\
\lesssim & \left.\|q\|_{H^{1}}\|v\|_{H^{1.75}}^{2}\left\|\partial_{t} v\right\|_{H^{1.5}}\right|_{t}+\left.\|q\|_{H^{1}}\|v\|_{H^{1.75}}^{2}\left\|\partial_{t} v\right\|_{H^{1.5}}\right|_{0} \\
& \quad+\int_{0}^{t}\left\|\partial_{t} q\right\|_{H^{1}}\|v\|_{H^{2}}\|v\|_{H^{1.5}}\left\|\partial_{t} v\right\|_{H^{1.5}}+\int_{0}^{t}\|q\|_{H^{1}}\left\|\partial_{t} v\right\|_{H^{1.5}}\|v\|_{H^{2}}\left\|\partial_{t} v\right\|_{H^{1.5}} \\
\lesssim & P_{0}+\left.\|q\|_{H^{1}}\|v\|_{H^{1.5}}^{(3-2 \nu) /(2-\nu)}\|v\|_{H^{2.5-\nu / 2}}^{1 /(2-\nu)}\left\|\partial_{t} v\right\|_{H^{1.5}}\right|_{t}+\int_{0}^{t} P,
\end{aligned}
$$

where we used $\|v\|_{H^{1.75}} \lesssim\|v\|_{H^{1.5}}^{(3-2 \nu) /(4-2 \nu)}\|v\|_{H^{2.5-\nu / 2}}^{1 /(4-2 \nu)}$ in the last step. On the other hand, the right side of (5.9) without $L$ is bounded by

$$
\begin{aligned}
& \|q\|_{H^{1.5+\delta_{0}}}\left\|\partial_{t} v\right\|_{H^{1}}^{2}+\|q(0)\|_{H^{1.5+\delta_{0}}}\left\|\partial_{t} v(0)\right\|_{H^{1}}^{2} \\
& \quad+\int_{0}^{t}\left(\left\|\partial_{t} q\right\|_{H^{1}}\|\eta\|_{H^{2}}\left\|\partial_{t} v\right\|_{H^{1.5}}^{2}+\|q\|_{H^{1}}\|v\|_{H^{2}}\left\|\partial_{t} v\right\|_{H^{1.5}}^{2}\right)+\|q\|_{H^{1.5+\delta_{0}}}\left\|\partial_{t} v\right\|_{H^{1}}^{2} \int_{0}^{t}\|v\|_{H^{2.5+\delta_{0}}} \\
& \quad \lesssim P_{0}+\|q\|_{H^{1}}^{\left(2-\nu-2 \delta_{0}\right) /(3-\nu)}\|q\|_{H^{2.5-\nu / 2}}^{\left(1+2 \delta_{0}\right) /(3-\nu)}\left\|\partial_{t} v\right\|_{L^{2}}^{2 / 3}\left\|\partial_{t} v\right\|_{H^{1.5}}^{4 / 3}\left(1+\int_{0}^{t} P\right)+\int_{0}^{t} P
\end{aligned}
$$

where $\delta_{0}>0$ is arbitrarily small. Note that the second term on the right side of (8.4) is an upper bound for both the first and the fourth terms on the left. Therefore, we conclude

$$
\begin{aligned}
\int_{0}^{t} J_{1} \lesssim P_{0} & +\|q\|_{H^{1}}^{\left(2-\nu-2 \delta_{0}\right) /(3-\nu)}\|q\|_{H^{2.5-\nu / 2}}^{\left(1+2 \delta_{0}\right) /(3-\nu)}\left\|\partial_{t} v\right\|_{L^{2}}^{2 / 3}\left\|\partial_{t} v\right\|_{H^{1.5}}^{4 / 3}\left(1+\int_{0}^{t} P\right) \\
& +\left.\|q\|_{H^{1}}\|v\|_{H^{1.5}}^{(3-2 \nu) /(2-\nu)}\|v\|_{H^{2.5-\nu / 2}}^{1 /(2-\nu)}\left\|\partial_{t} v\right\|_{H^{1.5}}\right|_{t}+\int_{0}^{t} P .
\end{aligned}
$$


Treatment of $J_{2}$ : Now we bound $\int_{0}^{t} J_{2}=2 \int_{0}^{t} \int_{\Omega} \partial_{t} a^{\mu \alpha} \partial_{t} q \partial_{\mu} \partial_{t}^{2} v_{\alpha}$. Using integration by parts in $x_{\mu}$ and the Piola identity (4.5), we get

$$
\int_{0}^{t} J_{2}=-2 \int_{0}^{t} \int_{\Omega} \partial_{t} a^{\mu \alpha} \partial_{t} \partial_{\mu} q \partial_{t}^{2} v_{\alpha}+2 \int_{0}^{t} \int_{\Gamma_{1}} \partial_{t} a^{\mu \alpha} \partial_{t} q \partial_{t}^{2} v_{\alpha} N_{\mu}=\int_{0}^{t} J_{21}+\int_{0}^{t} J_{22} .
$$

The first term is estimated using Hölder inequality as

$$
\int_{0}^{t} J_{21} \lesssim \int_{0}^{t}\left\|\partial_{t} a\right\|_{H^{1.5+\delta_{0}}}\left\|\partial_{t} q\right\|_{H^{1}}\left\|\partial_{t}^{2} v\right\|_{L^{2}} \lesssim \int_{0}^{t} P
$$

For the second term in (8.5), we integrate by parts in $t$, leading to

$$
\begin{aligned}
\int_{0}^{t} J_{22} & =\left.2 \int_{\Gamma_{1}} \partial_{t} a^{\mu \alpha} \partial_{t} q \partial_{t} v_{\alpha} N_{\mu}\right|_{0} ^{t}-2 \int_{0}^{t} \int_{\Gamma_{1}} \partial_{t}^{2} a^{\mu \alpha} \partial_{t} q \partial_{t} v_{\alpha} N_{\mu}-2 \int_{0}^{t} \int_{\Gamma_{1}} \partial_{t} a^{\mu \alpha} \partial_{t}^{2} q \partial_{t} v_{\alpha} N_{\mu} \\
& =J_{221}+\int_{0}^{t} J_{222}+\int_{0}^{t} J_{223} .
\end{aligned}
$$

For the pointwise in time term, we have

$$
\begin{aligned}
\left.J_{221}\right|_{t} & \lesssim\left\|\partial_{t} a\right\|_{H^{1 / 2}\left(\Gamma_{1}\right)}\left\|\partial_{t} q\right\|_{H^{1 / 2}\left(\Gamma_{1}\right)}\left\|\partial_{t} v\right\|_{L^{2}\left(\Gamma_{1}\right)} \\
& \lesssim\left\|\partial_{t} a\right\|_{H^{1}}\left\|\partial_{t} q\right\|_{H^{1}}\left\|\partial_{t} v\right\|_{H^{1 / 2+\delta_{0}}} \lesssim\|v\|_{H^{2}}\left\|\partial_{t} q\right\|_{H^{1}}\left\|\partial_{t} v\right\|_{H^{1 / 2+\delta_{0}}} \\
& \lesssim\|v\|_{H^{1.5}}^{(1-\nu) /(2-\nu)}\|v\|_{H^{2.5-\nu / 2}}^{1 /(2-\nu)}\left\|\partial_{t} q\right\|_{H^{1}}\left\|\partial_{t} v\right\|_{L^{2}}^{\left(2-2 \delta_{0}\right) / 3}\left\|\partial_{t} v\right\|_{H^{1.5}}^{\left(1+2 \delta_{0}\right) / 3}
\end{aligned}
$$

We emphasize that (2.5) and (2.6) should not be used to treat $J_{222}$. Instead, we write

$$
\int_{0}^{t} J_{222}=-2 \int_{0}^{t} \int_{\Gamma_{1}} \partial_{t}^{2} a^{\mu \alpha} \partial_{t} q \partial_{t} v_{\alpha} N_{\mu}=-2 \int_{0}^{t} \int_{\Gamma_{1}} \partial_{t}^{2} a^{3 \alpha} \partial_{t} q \partial_{t} v_{\alpha}
$$

From [35], recall the formula for the third row of the matrix $a$, which reads

$$
a^{3 \cdot}=\left[\partial_{1} \eta^{2} \partial_{2} \eta^{3}-\partial_{2} \eta^{2} \partial_{1} \eta^{3}, \quad \partial_{2} \eta^{1} \partial_{1} \eta^{3}-\partial_{1} \eta^{1} \partial_{2} \eta^{3}, \quad \partial_{1} \eta^{1} \partial_{2} \eta^{2}-\partial_{2} \eta^{1} \partial_{1} \eta^{2}\right]
$$

It is essential that only tangential derivatives appear in each entry. Therefore, for all $\alpha=1,2,3$,

$$
\begin{aligned}
\left\|\partial_{t}^{2} a^{3 \alpha}\right\|_{L^{2}\left(\Gamma_{1}\right)} & \lesssim\|\bar{\partial} \eta\|_{H^{1+\delta_{0}}\left(\Gamma_{1}\right)}\left\|\bar{\partial} \partial_{t} v\right\|_{L^{2}\left(\Gamma_{1}\right)}+\|\bar{\partial} v\|_{H^{0.5}\left(\Gamma_{1}\right)}^{2} \\
& \lesssim\|\eta\|_{H^{2+\delta_{0}}\left(\Gamma_{1}\right)}\left\|\partial_{t} v\right\|_{H^{1}\left(\Gamma_{1}\right)}+\|v\|_{H^{1.5}\left(\Gamma_{1}\right)}^{2} \\
& \lesssim\|\eta\|_{H^{2.5+\delta_{0}}}\left\|\partial_{t} v\right\|_{H^{1.5}}+\|v\|_{H^{2}}^{2} \lesssim\left\|\partial_{t} v\right\|_{H^{1.5}}+\|v\|_{H^{2}}^{2} \lesssim P .
\end{aligned}
$$

Thus we have

$$
\int_{0}^{t} J_{222} \lesssim \int_{0}^{t}\left\|\partial_{t}^{2} a\right\|_{L^{2}\left(\Gamma_{1}\right)}\left\|\partial_{t} q\right\|_{H^{1 / 2}\left(\Gamma_{1}\right)}\left\|\partial_{t} v\right\|_{H^{1 / 2}\left(\Gamma_{1}\right)} \lesssim \int_{0}^{t}\left\|\partial_{t}^{2} a\right\|_{L^{2}\left(\Gamma_{1}\right)}\left\|\partial_{t} q\right\|_{H^{1}}\left\|\partial_{t} v\right\|_{H^{1}} \leq \int_{0}^{t} P
$$


using (8.6) and the trace inequality. Lastly, we consider $J_{223}$, for which we use (2.5) and (2.6):

$$
\begin{aligned}
\int_{0}^{t} J_{223}= & 2 \int_{0}^{t} \int_{\Gamma_{1}} a^{\mu \beta} \partial_{\lambda} v_{\beta} a^{\lambda \alpha} \partial_{t}^{2} q \partial_{t} v_{\alpha} N_{\mu} \\
= & 2 \int_{0}^{t} \int_{\Gamma_{1}} \partial_{t}^{2}\left(N_{\mu} a^{\mu \beta} q\right) \partial_{\lambda} v_{\beta} a^{\lambda \alpha} \partial_{t} v_{\alpha}-2 \int_{0}^{t} \int_{\Gamma_{1}} \partial_{t}^{2} a^{\mu \beta} q \partial_{\lambda} v_{\beta} a^{\lambda \alpha} \partial_{t} v_{\alpha} N_{\mu} \\
& -4 \int_{0}^{t} \int_{\Gamma_{1}} \partial_{t} a^{\mu \beta} \partial_{t} q \partial_{\lambda} v_{\beta} a^{\lambda \alpha} \partial_{t} v_{\alpha} N_{\mu} \\
= & 2 \int_{0}^{t} \int_{\Gamma_{1}} \partial_{t}^{2}\left(\partial_{i}\left(\sqrt{g} g^{i j} \partial_{j} \eta^{\beta}\right)\right) \partial_{\lambda} v_{\beta} a^{\lambda \alpha} \partial_{t} v_{\alpha}-2 \int_{0}^{t} \int_{\Gamma_{1}} \partial_{t}^{2} a^{3 \beta} q \partial_{\lambda} v_{\beta} a^{\lambda \alpha} \partial_{t} v_{\alpha} \\
& -4 \int_{0}^{t} \int_{\Gamma_{1}} \partial_{t} a^{3 \beta} \partial_{t} q \partial_{\lambda} v_{\beta} a^{\lambda \alpha} \partial_{t} v_{\alpha} \\
= & -2 \int_{0}^{t} \int_{\Gamma_{1}} \partial_{t}^{2}\left(\sqrt{g} g^{i j} \partial_{j} \eta^{\beta}\right) \partial_{i}\left(\partial_{\lambda} v_{\beta} a^{\lambda \alpha} \partial_{t} v_{\alpha}\right) \\
& -2 \int_{0}^{t} \int_{\Gamma_{1}} \partial_{t}^{2} a^{3 \beta} q \partial_{\lambda} v_{\beta} a^{\lambda \alpha} \partial_{t} v_{\alpha}-4 \int_{0}^{t} \int_{\Gamma_{1}} \partial_{t} a^{3 \beta} \partial_{t} q \partial_{\lambda} v_{\beta} a^{\lambda \alpha} \partial_{t} v_{\alpha} \\
= & \int_{0}^{t} J_{2231}+\int_{0}^{t} J_{2232}+\int_{0}^{t} J_{2233} .
\end{aligned}
$$

The term $\int_{0}^{t} J_{2231}$ may now be estimated with $\int_{0}^{t} P$ by simply expanding. For the second term, we use (8.6), after which it is also bounded by $\int_{0}^{t} P$. The third term is also bounded directly, and thus $\int_{0}^{t} J_{223} \lesssim \int_{0}^{t} P$. Collecting all the inequalities, we get

$$
\int_{0}^{t} J_{2} \lesssim P_{0}+\|v\|_{H^{1.5}}^{(1-\nu) /(2-\nu)}\|v\|_{H^{2.5-\nu / 2}}^{1 /(2-\nu)}\left\|\partial_{t} q\right\|_{H^{1}}\left\|\partial_{t} v\right\|_{L^{2}}^{\left(2-2 \delta_{0}\right) / 3}\left\|\partial_{t} v\right\|_{H^{1.5}}^{\left(1+2 \delta_{0}\right) / 3}+\int_{0}^{t} P .
$$

Treatment of $J_{3}$ : Here we estimate $J_{3}=\int_{\Omega} a^{\mu \alpha} \partial_{t}^{2} q \partial_{t}^{2} \partial_{\mu} v_{\alpha}$. Using (2.3) and (2.5), the term $J_{3}$ can be expressed as

$$
\begin{aligned}
J_{3}= & -\int_{\Omega} \partial_{t}^{2} a^{\mu \alpha} \partial_{t}^{2} q \partial_{\mu} v_{\alpha}-2 \int_{\Omega} \partial_{t} a^{\mu \alpha} \partial_{t}^{2} q \partial_{\mu} \partial_{t} v_{\alpha} \\
= & \int_{\Omega} a^{\mu \beta} \partial_{\lambda} \partial_{t} v_{\beta} a^{\lambda \alpha} \partial_{t}^{2} q \partial_{\mu} v_{\alpha}+\int_{\Omega} \partial_{t}\left(a^{\mu \beta} a^{\lambda \alpha}\right) \partial_{\lambda} v_{\beta} \partial_{t}^{2} q \partial_{\mu} v_{\alpha} \\
& \quad-2 \int_{\Omega} \partial_{t} a^{\mu \alpha} \partial_{t}^{2} q \partial_{\mu} \partial_{t} v_{\alpha}=J_{31}+J_{32}+J_{33} .
\end{aligned}
$$

To treat the term $J_{31}$, we integrate by parts in $x_{\lambda}$ obtaining

$$
\begin{aligned}
J_{31}= & -\int_{\Omega} \partial_{\lambda} a^{\mu \beta} \partial_{t} v_{\beta} a^{\lambda \alpha} \partial_{t}^{2} q \partial_{\mu} v_{\alpha}-\int_{\Omega} a^{\mu \beta} \partial_{t} v_{\beta} a^{\lambda \alpha} \partial_{\lambda} \partial_{t}^{2} q \partial_{\mu} v_{\alpha} \\
& \quad-\int_{\Omega} a^{\mu \beta} \partial_{t} v_{\beta} a^{\lambda \alpha} \partial_{t}^{2} q \partial_{\lambda} \partial_{\mu} v_{\alpha}+\int_{\Gamma_{1}} a^{\mu \beta} \partial_{t} v_{\beta} a^{\lambda \alpha} \partial_{t}^{2} q \partial_{\mu} v_{\alpha} N_{\lambda} \\
= & J_{311}+J_{312}+J_{313}+J_{314}
\end{aligned}
$$

where we used (4.5). Integrating in time the first term and then treating it by integration by parts in time, we get

$$
\begin{aligned}
\int_{0}^{t} J_{311}=- & \left.\int_{\Omega} \partial_{\lambda} a^{\mu \beta} \partial_{t} v_{\beta} a^{\lambda \alpha} \partial_{t} q \partial_{\mu} v_{\alpha}\right|_{0} ^{t}+\int_{0}^{t} \int_{\Omega} \partial_{t}\left(\partial_{\lambda} a^{\mu \beta} \partial_{t} v_{\beta} a^{\lambda \alpha}\right) \partial_{t} q \partial_{\mu} v_{\alpha} \\
& +\int_{0}^{t} \int_{\Omega} \partial_{\lambda} a^{\mu \beta} \partial_{t} v_{\beta} a^{\lambda \alpha} \partial_{t} q \partial_{\mu} \partial_{t} v_{\alpha} .
\end{aligned}
$$


The pointwise in time term in the above sum may be bounded as

$$
-\left.\int_{\Omega} \partial_{\lambda} a^{\mu \beta} \partial_{t} v_{\beta} a^{\lambda \alpha} \partial_{t} q \partial_{\mu} v_{\alpha}\right|_{t} \lesssim\|a\|_{H^{1.5}}\left\|\partial_{t} v\right\|_{H^{\nu / 2}}\left\|\partial_{t} q\right\|_{H^{1}}\|v\|_{H^{2.5-\nu / 2}} \lesssim\left\|\partial_{t} v\right\|_{H^{\nu / 2}}\left\|\partial_{t} q\right\|_{H^{1}}\|v\|_{H^{2.5-\nu / 2}}
$$

by Lemma 3.1(ii), and we obtain

$$
\int_{0}^{t} J_{311} \lesssim P_{0}+\left\|\partial_{t} v\right\|_{L^{2}}^{1-\nu / 3}\left\|\partial_{t} v\right\|_{H^{1.5}}^{\nu / 3}\left\|\partial_{t} q\right\|_{H^{1}}\|v\|_{H^{2.5-\nu / 2}}+\int_{0}^{t} P
$$

where we used Lemma 3.1(ii). Similarly, using the divergence condition (2.3), we have $a^{\lambda \alpha} \partial_{\lambda} \partial_{\mu} v_{\alpha}=-\partial_{\mu} a^{\lambda \alpha} \partial_{\lambda} v_{\alpha}$, and the third term in (8.8) can be rewritten as $J_{313}=\int_{\Omega} a^{\mu \beta} \partial_{t} v_{\beta} \partial_{\mu} a^{\lambda \alpha} \partial_{t}^{2} q \partial_{\lambda} v_{\alpha}$. Note that it has the same structure as $J_{311}$ and it thus satisfies the same estimate.

In the term $J_{312}$, we integrate by parts in time, obtaining

$$
\begin{aligned}
\int_{0}^{t} J_{312}=- & \left.\int_{\Omega} a^{\mu \beta} \partial_{t} v_{\beta} a^{\lambda \alpha} \partial_{\lambda} \partial_{t} q \partial_{\mu} v_{\alpha}\right|_{0} ^{t}+\int_{0}^{t} \int_{\Omega} \partial_{t}\left(a^{\mu \beta} \partial_{t} v_{\beta} a^{\lambda \alpha}\right) \partial_{\lambda} \partial_{t} q \partial_{\mu} v_{\alpha} \\
& +\int_{0}^{t} \int_{\Omega} a^{\mu \beta} \partial_{t} v_{\beta} a^{\lambda \alpha} \partial_{\lambda} \partial_{t} q \partial_{\mu} \partial_{t} v_{\alpha} .
\end{aligned}
$$

The pointwise in term satisfies

$$
\begin{gathered}
-\left.\int_{\Omega} a^{\mu \beta} \partial_{t} v_{\beta} a^{\lambda \alpha} \partial_{\lambda} \partial_{t} q \partial_{\mu} v_{\alpha}\right|_{0} ^{t} \lesssim\left\|\partial_{t} v\right\|_{H^{\nu / 2}}\left\|\partial_{t} q\right\|_{H^{1}}\|v\|_{H^{2.5-\nu / 2}}+\left.\left\|\partial_{t} v\right\|_{H^{\nu / 2}}\left\|\partial_{t} q\right\|_{H^{1}}\|v\|_{H^{2.5-\nu / 2}}\right|_{0} \\
\lesssim P_{0}+\left\|\partial_{t} v\right\|_{L^{2}}^{1-\nu / 3}\left\|\partial_{t} v\right\|_{H^{1.5}}^{\nu / 3}\left\|\partial_{t} q\right\|_{H^{1}}\|v\|_{H^{2.5-\nu / 2}}
\end{gathered}
$$

where, in particular, we used Lemma 3.1(ii). (Note that this has the same upper bound as in (8.9).) Therefore,

$$
\int_{0}^{t} J_{312} \lesssim P_{0}+\left\|\partial_{t} v\right\|_{L^{2}}^{1-\nu / 3}\left\|\partial_{t} v\right\|_{H^{1.5}}^{\nu / 3}\left\|\partial_{t} q\right\|_{H^{1}}\|v\|_{H^{2.5-\nu / 2}}+\int_{0}^{t} P
$$

The boundary term $J_{314}$ can be expressed as

$$
\begin{aligned}
\int_{0}^{t} J_{314}= & \int_{0}^{t} \int_{\Gamma_{1}} a^{\mu \beta} \partial_{t} v_{\beta} a^{\lambda \alpha} \partial_{t}^{2} q \partial_{\mu} v_{\alpha} N_{\lambda} \\
= & \int_{0}^{t} \int_{\Gamma_{1}} \partial_{t}^{2}\left(N_{\lambda} a^{\lambda \alpha} q\right) a^{\mu \beta} \partial_{t} v_{\beta} \partial_{\mu} v_{\alpha}-\int_{0}^{t} \int_{\Gamma_{1}} \partial_{t}^{2} a^{\lambda \alpha} q a^{\mu \beta} \partial_{t} v_{\beta} \partial_{\mu} v_{\alpha} N_{\lambda} \\
& -2 \int_{0}^{t} \int_{\Gamma_{1}} \partial_{t} a^{\lambda \alpha} \partial_{t} q a^{\mu \beta} \partial_{t} v_{\beta} \partial_{\mu} v_{\alpha} N_{\lambda} .
\end{aligned}
$$

Note that all three terms have the same structure as the three terms in (8.7) and are treated analogously, leading to the same upper bounds.

The term $J_{32}$ is treated by using integration by parts in time (and no integration by parts in space). Since all the terms are treated in a straight-forward way, we only estimate the pointwise in time term, which equals

$$
\begin{aligned}
\int_{\Omega} \partial_{t}\left(a^{\mu \beta} a^{\lambda \alpha}\right) \partial_{\lambda} v_{\beta} \partial_{t} q \partial_{\mu} v_{\alpha} \lesssim\left\|\partial_{t} a\right\|_{H^{1}}\|v\|_{H^{1.5}}^{2}\left\|\partial_{t} q\right\|_{H^{1}} \\
\quad \lesssim\|v\|_{H^{2}}\|v\|_{H^{1.5}}^{2}\left\|\partial_{t} q\right\|_{H^{1}} \lesssim\|v\|_{H^{1.5}}^{(1-\nu) /(2-\nu)}\|v\|_{H^{2.5-\nu / 2}}^{1 /(2-\nu)}\|v\|_{H^{1.5}}^{2}\left\|\partial_{t} q\right\|_{H^{1}}
\end{aligned}
$$

by (4.11).

It remains to consider $J_{33}$. We first integrate by parts in $x_{\mu}$ leading to

$$
\begin{aligned}
\int_{0}^{t} J_{33} & =-2 \int_{0}^{t} \int_{\Omega} \partial_{t} a^{\mu \alpha} \partial_{t}^{2} q \partial_{\mu} \partial_{t} v_{\alpha}=2 \int_{0}^{t} \int_{\Omega} \partial_{t} a^{\mu \alpha} \partial_{t}^{2} \partial_{\mu} q \partial_{t} v_{\alpha}-2 \int_{0}^{t} \int_{\Gamma_{1}} \partial_{t} a^{\mu \alpha} \partial_{t}^{2} q \partial_{t} v_{\alpha} N_{\mu} \\
& =\int_{0}^{t} J_{331}+\int_{0}^{t} J_{332} .
\end{aligned}
$$


The second term is identical to $\int_{0}^{t} J_{223}$. For $J_{331}$, we integrate by parts in time. The integrated in time terms are controlled by $\int_{0}^{t} P$, while the pointwise in time term evaluated at $t$ reads

$$
\left.2 \int_{\Omega} \partial_{t} a^{\mu \alpha} \partial_{t} \partial_{\mu} q \partial_{t} v_{\alpha}\right|_{t} \lesssim\left\|\partial_{t} a\right\|_{H^{1.5-\nu / 2}}\left\|\partial_{t} \nabla q\right\|_{L^{2}}\left\|\partial_{t} v\right\|_{H^{\nu / 2}}
$$

Note that this is the same upper bound as in (8.10).

Treatment of $J_{4}$ : It only remains to consider the boundary term $J_{4}$, in which case we use (6.4) with $\mathcal{E}=\partial_{t}$. Thus

$$
\begin{aligned}
& J_{4} \lesssim- \frac{1}{2} \frac{d}{d t} \int_{\Gamma_{1}} \sqrt{g} g^{i j} \Pi_{\lambda}^{\mu} \partial_{t} \partial_{j} v^{\lambda} \Pi_{\mu}^{\alpha} \partial_{t} \partial_{i} v_{\alpha}+\frac{d}{d t} \int_{\Gamma_{1}} \tilde{Q}_{\mu \lambda}^{i}(\bar{\partial} \eta) \bar{\partial}^{2} \eta \partial_{t} v^{\mu} \partial_{i} \partial_{t} v^{\lambda} \\
&-\int_{\Gamma_{1}}\left(\partial_{t}\left(\sqrt{g} g^{i j} \Pi_{\lambda}^{\alpha} \partial_{j} v^{\lambda}\right)-\sqrt{g} g^{i j} \Pi_{\lambda}^{\alpha} \partial_{t} \partial_{j} v^{\lambda}\right) \partial_{i} \partial_{t}^{2} v_{\alpha} \\
&-\int_{\Gamma_{1}}\left(\partial_{t}\left(\sqrt{g}\left(g^{i j} g^{k l}-g^{l j} g^{i k}\right) \partial_{j} \eta^{\alpha} \partial_{k} \eta_{\lambda} \partial_{l} v^{\lambda}\right)\right. \\
&\left.-\sqrt{g}\left(g^{i j} g^{k l}-g^{l j} g^{i k}\right) \partial_{j} \eta^{\alpha} \partial_{k} \eta_{\lambda} \partial_{l} \partial_{t} v^{\lambda}\right) \partial_{i} \partial_{t}^{2} v_{\alpha} \\
&+P\left(\|\eta\|_{H^{2.5+\delta_{0}}}\right)\left(\|v\|_{H^{2.5+\delta_{0}}}+1\right)\left\|\partial_{t} v\right\|_{H^{1.5}}^{2}
\end{aligned}
$$

Note that the last term is dominated by $P$. Therefore,

$$
\begin{aligned}
\int_{0}^{t} J_{4} \lesssim & \left.\frac{1}{2} \int_{\Gamma_{1}} \sqrt{g} g^{i j} \Pi_{\lambda}^{\mu} \partial_{t} \partial_{j} v^{\lambda} \Pi_{\mu}^{\alpha} \partial_{t} \partial_{i} v_{\alpha}\right|_{0} ^{t}+\left.\int_{\Gamma_{1}} \tilde{Q}_{\mu \lambda}^{i}(\bar{\partial} \eta) \bar{\partial}^{2} \eta \partial_{t} v^{\mu} \partial_{i} \partial_{t} v^{\lambda}\right|_{0} ^{t} \\
& -\int_{0}^{t} \int_{\Gamma_{1}} \partial_{t}\left(\sqrt{g} g^{i j} \Pi_{\lambda}^{\alpha}\right) \partial_{j} v^{\lambda} \partial_{i} \partial_{t}^{2} v_{\alpha} \\
& -\int_{0}^{t} \int_{\Gamma_{1}} \partial_{t}\left(\sqrt{g}\left(g^{i j} g^{k l}-g^{l j} g^{i k}\right) \partial_{j} \eta^{\alpha} \partial_{k} \eta_{\lambda}\right) \partial_{l} v^{\lambda} \partial_{i} \partial_{t}^{2} v_{\alpha}+\int_{0}^{t} P \\
= & J_{41}+J_{42}+\int_{0}^{t} J_{43}+\int_{0}^{t} J_{44}+\int_{0}^{t} P .
\end{aligned}
$$

As for $I_{41}$ in the previous section, the first term $J_{41}$ is the coercive term leading to the second term on the left side of (8.2) by simply using (7.7). The second term $J_{42}$ is bounded in (6.7) as $J_{42} \lesssim P_{0}+\int_{0}^{t} P+\epsilon_{0}\left\|\partial_{t} v\right\|_{H^{1.5}}^{2}$. For $J_{43}$ and $J_{44}$, we integrate by parts in time, yielding

$$
\begin{aligned}
\int_{0}^{t} J_{43}+\int_{0}^{t} J_{44}=- & \left.\int_{\Gamma_{1}} \partial_{t}\left(\sqrt{g} g^{i j} \Pi_{\lambda}^{\alpha}\right) \partial_{j} v^{\lambda} \partial_{i} \partial_{t} v_{\alpha}\right|_{0} ^{t}+\int_{0}^{t} \int_{\Gamma_{1}} \partial_{t}^{2}\left(\sqrt{g} g^{i j} \Pi_{\lambda}^{\alpha}\right) \partial_{j} v^{\lambda} \partial_{i} \partial_{t} v_{\alpha} \\
& +\int_{0}^{t} \int_{\Gamma_{1}} \partial_{t}\left(\sqrt{g} g^{i j} \Pi_{\lambda}^{\alpha}\right) \partial_{j} \partial_{t} v^{\lambda} \partial_{i} \partial_{t} v_{\alpha} \\
& -\left.\int_{\Gamma_{1}} \partial_{t}\left(\sqrt{g}\left(g^{i j} g^{k l}-g^{l j} g^{i k}\right) \partial_{j} \eta^{\alpha} \partial_{k} \eta_{\lambda}\right) \partial_{l} v^{\lambda} \partial_{i} \partial_{t} v_{\alpha}\right|_{0} ^{t} \\
& +\int_{0}^{t} \int_{\Gamma_{1}} \partial_{t}^{2}\left(\sqrt{g}\left(g^{i j} g^{k l}-g^{l j} g^{i k}\right) \partial_{j} \eta^{\alpha} \partial_{k} \eta_{\lambda}\right) \partial_{l} v^{\lambda} \partial_{i} \partial_{t} v_{\alpha} \\
& +\int_{0}^{t} \int_{\Gamma_{1}} \partial_{t}\left(\sqrt{g}\left(g^{i j} g^{k l}-g^{l j} g^{i k}\right) \partial_{j} \eta^{\alpha} \partial_{k} \eta_{\lambda}\right) \partial_{l} \partial_{t} v^{\lambda} \partial_{i} \partial_{t} v_{\alpha}
\end{aligned}
$$

whence

$$
\begin{aligned}
\int_{0}^{t} J_{43}+\int_{0}^{t} J_{44} & \lesssim P_{0}+\|v\|_{H^{2}}^{2}\left\|\partial_{t} v\right\|_{H^{1.5}}+\int_{0}^{t} P \\
& \lesssim P_{0}+\|v\|_{H^{1.5}}^{2(1-\nu) /(2-\nu)}\|v\|_{H^{2.5-\nu / 2}}^{2 /(2-\nu)}\left\|\partial_{t} v\right\|_{H^{1.5}}+\int_{0}^{t} P
\end{aligned}
$$


after a short calculation. Note that both pointwise in time terms in (8.11) are estimated by the second term on the far right side of (8.12). The proof of the lemma is thus complete.

\section{Div-Curl estimates AND the CAUChy INVARIANCE}

In this section, we use the Cauchy invariance property and the div-curl estimates to the norms $\|v\|_{H^{2.5-\nu / 2}}$, $\|v\|_{H^{3-\nu}}$, and $\left\|\partial_{t} v\right\|_{H^{1.5}}$ in terms of $\left\|v^{3}\right\|_{H^{2-\nu / 2}\left(\Gamma_{1}\right)},\left\|v^{3}\right\|_{H^{2.5-\nu}\left(\Gamma_{1}\right)}$, and $\left\|\partial_{t} v^{3}\right\|_{H^{1}\left(\Gamma_{1}\right)}$, respectively.

We summarize the resulting inequalities in the following statement.

Lemma 9.1. For the velocity $v$, we have

$$
\|v\|_{H^{2.5-\nu / 2}} \lesssim P_{0}+\left\|v^{3}\right\|_{H^{2-\nu / 2}\left(\Gamma_{1}\right)}
$$

and

$$
\|v\|_{H^{3-\nu}} \lesssim P_{0}+\left\|v^{3}\right\|_{H^{2.5-\nu}\left(\Gamma_{1}\right)}
$$

while for the derivative $\partial_{t} v$, we have

$$
\left\|\partial_{t} v\right\|_{H^{1.5}} \lesssim\|v\|_{H^{2}}^{2}+\left\|\partial_{t} v^{3}\right\|_{H^{1}\left(\Gamma_{1}\right)} .
$$

Proof of Lemma 9.1. First, let $p_{0} \in\{1.5-\nu / 2,2-\nu\}$. By the Cauchy invariance property

$$
\epsilon^{\alpha \beta \gamma} \partial_{\beta} v^{\mu} \partial_{\gamma} \eta_{\mu}=\left(\operatorname{curl} v_{0}\right)^{\alpha}
$$

(cf. [58]), we obtain

$$
(\operatorname{curl} v)^{\alpha}=\epsilon^{\alpha \beta \gamma} \partial_{\beta} v_{\gamma}=\epsilon^{\alpha \beta \gamma} \partial_{\beta} v^{\mu}\left(\delta_{\gamma \mu}-\partial_{\gamma} \eta_{\mu}\right)+\left(\operatorname{curl} v_{0}\right)^{\alpha} .
$$

Similarly, from the divergence condition (2.3), we get

$$
\operatorname{div} v=\left(\delta^{\alpha \beta}-a^{\alpha \beta}\right) \partial_{\alpha} v_{\beta} .
$$

Using the elliptic estimate

$$
\|X\|_{H^{s}} \lesssim\|\operatorname{curl} X\|_{H^{s-1}}+\|\operatorname{div} X\|_{H^{s-1}}+\|X \cdot N\|_{H^{s-0.5}\left(\Gamma_{1} \cup \Gamma_{0}\right)}, \quad s \geq 1
$$

([16, 21, 25]) along with (2.7), (9.5), and (9.6), we arrive at

$$
\|v\|_{H^{p_{0}+1}} \lesssim\|\nabla \eta-I\|_{H^{2-\nu}}\|\nabla v\|_{H^{p_{0}}}+\|a-I\|_{H^{2-\nu}}\|\nabla v\|_{H^{p_{0}}}+\left\|\operatorname{curl} v_{0}\right\|_{H^{p_{0}}}+\left\|v^{3}\right\|_{H^{p_{0}+0.5}\left(\Gamma_{1}\right)} .
$$

Assuming that the time $T>0$ is sufficiently small as in Lemma 3.1(iv), the first two terms on the right side of (9.8) may be dominated by the left, and we obtain

$$
\|v\|_{H^{p_{0}+1}} \lesssim\left\|\operatorname{curl} v_{0}\right\|_{H^{p_{0}}}+\left\|v^{3}\right\|_{H^{p_{0}+0.5}\left(\Gamma_{1}\right)}, \quad p_{0} \in\{1.5-\nu / 2,2-\nu\} .
$$

Therefore, we obtain (9.1) and (9.2).

Next, we apply the Cauchy invariance to $\partial_{t} v$, i.e.,

$$
\epsilon^{\alpha \beta \gamma} \partial_{\beta} \partial_{t} v^{\mu} \partial_{\gamma} \eta_{\mu}=-\epsilon^{\alpha \beta \gamma} \partial_{\beta} v^{\mu} \partial_{\gamma} v_{\mu}
$$

obtained by differentiating (9.4) in $t$, which we may rewrite as

$$
\begin{aligned}
\left(\operatorname{curl} \partial_{t} v\right)^{\alpha} & =\epsilon^{\alpha \beta \gamma} \partial_{\beta} \partial_{t} v_{\gamma}=\epsilon^{\alpha \beta \gamma} \partial_{\beta} \partial_{t} v^{\mu}\left(\delta_{\gamma \mu}-\partial_{\gamma} \eta_{\mu}\right)+\epsilon^{\alpha \beta \gamma} \partial_{\beta} \partial_{t} v^{\mu} \partial_{\gamma} \eta_{\mu} \\
& =\epsilon^{\alpha \beta \gamma} \partial_{\beta} \partial_{t} v^{\mu}\left(\delta_{\gamma \mu}-\partial_{\gamma} \eta_{\mu}\right)-\epsilon^{\alpha \beta \gamma} \partial_{\beta} v^{\mu} \partial_{\gamma} v_{\mu}
\end{aligned}
$$

using (9.9) in the last step. On the other hand, the divergence condition for $\partial_{t} v$ may be rewritten as $\partial^{\beta} \partial_{t} v_{\beta}=$ $\left(\delta^{\alpha \beta}-a^{\alpha \beta}\right) \partial_{\alpha} \partial_{t} v_{\beta}-\partial_{t} a^{\alpha \beta} \partial_{\alpha} v_{\beta}$. Using the div-curl elliptic estimate (9.7) with $X=\partial_{t} v$ and $s=1.5$, we get

$$
\begin{aligned}
\left\|\partial_{t} v\right\|_{H^{1.5}} \lesssim\|\nabla \eta-I\|_{H^{2-\nu}}\left\|\nabla \partial_{t} v\right\|_{H^{0.5}}+\|a-I\|_{H^{2-\nu}}\left\|\nabla \partial_{t} v\right\|_{H^{0.5}} & \\
& \quad+\|v\|_{H^{2}}^{2}+\left\|\partial_{t} a\right\|_{H^{1}}\|v\|_{H^{2}}+\left\|\partial_{t} v^{3}\right\|_{H^{1}\left(\Gamma_{1}\right)} \\
\lesssim & \|\nabla \eta-I\|_{H^{2-\nu}}\left\|\nabla \partial_{t} v\right\|_{H^{0.5}}+\|a-I\|_{H^{2-\nu}}\left\|\nabla \partial_{t} v\right\|_{H^{0.5}}+\|v\|_{H^{2}}^{2}+\left\|\partial_{t} v^{3}\right\|_{H^{1}\left(\Gamma_{1}\right)}
\end{aligned}
$$


and thus, if $T$ is sufficiently small as in Lemma 3.1(iv), we obtain (9.3).

\section{RELATION BETWEEN THE PROJECTION AND THE NORMAL COMPONENT OF $v$ AND $\partial_{t} v$}

In order to close the estimates, we need to connect the projections and the normal components of the vector fields $\left.v^{3}\right|_{\Gamma_{1}}$ and $\left.\partial_{t} v^{3}\right|_{\Gamma_{1}}$. We first address the comparison between $\Pi X$ and $X \cdot N$, where $X$ shall be chosen as certain derivative operators of $v$ and $\partial_{t} v$.

From (6.2), recall that $\Pi_{\alpha}^{\beta}=\delta_{\alpha}^{\beta}-g^{k l} \partial_{k} \eta^{\beta} \partial_{l} \eta_{\alpha}$. Therefore, $(\Pi X)^{3}=\Pi_{\alpha}^{3} X^{\alpha}=\delta_{\alpha}^{3} X^{\alpha}-g^{k l} \partial_{l} \eta_{\alpha} \partial_{k} \eta^{3} X^{\alpha}$, from where $X^{3}=(\Pi X)^{3}+g^{k l} \partial_{k} \eta_{\alpha} \partial_{l} \eta^{3} X^{\alpha}$. Using $\eta^{3}=\eta^{3}(0)+\int_{0}^{t} v^{3}=x^{3}+\int_{0}^{t} v^{3}$, and thus $\partial_{l} \eta^{3}=\int_{0}^{t} \partial_{l} v^{3}$, we get

$$
X^{3}=(\Pi X)^{3}+g^{k l} \partial_{k} \eta_{\alpha} X^{\alpha} \int_{0}^{t} \partial_{l} v^{3} .
$$

Applying the formula (10.1) with $X=\bar{\partial} \partial_{t} v$. From $\bar{\partial} \partial_{t} v^{3}=\left(\Pi \bar{\partial} \partial_{t} v\right)^{3}+g^{k l} \partial_{k} \eta_{\alpha} \bar{\partial} \partial_{t} v^{\alpha} \int_{0}^{t} \partial_{l} v^{3}$ we obtain

$$
\left\|\bar{\partial} \partial_{t} v^{3}\right\|_{L^{2}\left(\Gamma_{1}\right)} \lesssim\left\|\Pi \bar{\partial} \partial_{t} v\right\|_{L^{2}\left(\Gamma_{1}\right)}+\left\|g^{k l} \partial_{k} \eta_{\alpha} \bar{\partial} \partial_{t} v^{\alpha} \int_{0}^{t} \partial_{l} v^{3}\right\|_{L^{2}\left(\Gamma_{1}\right)} .
$$

The first term on the right side is estimated in Section 8. For the second term, we have

$$
\begin{gathered}
\left\|g^{k l} \partial_{k} \eta_{\alpha} \bar{\partial} \partial_{t} v^{\alpha} \int_{0}^{t} \partial_{l} v^{3}\right\|_{L^{2}\left(\Gamma_{1}\right)} \lesssim\left\|g^{-1} \bar{\partial} \eta\right\|_{L^{\infty}\left(\Gamma_{1}\right)}\left\|\bar{\partial} \partial_{t} v\right\|_{L^{2}\left(\Gamma_{1}\right)} \int_{0}^{t}\|\bar{\partial} v\|_{L^{\infty}\left(\Gamma_{1}\right)} \\
\lesssim Q\left(\|\eta\|_{\left.H^{2.5+\delta_{0}}\right)}\right)\left\|\partial_{t} v\right\|_{H^{1.5}} \int_{0}^{t}\|v\|_{H^{2.5+\delta_{0}}} \lesssim\left\|\partial_{t} v\right\|_{H^{1.5}} \int_{0}^{t}\|v\|_{H^{3-\nu}}
\end{gathered}
$$

where we used $\|\eta\|_{H^{2.5+\delta_{0}}} \lesssim 1$ from Lemma 3.1(i). Therefore, $\left\|\bar{\partial} \partial_{t} v^{3}\right\|_{L^{2}\left(\Gamma_{1}\right)} \lesssim\left\|\Pi \bar{\partial} \partial_{t} v\right\|_{L^{2}\left(\Gamma_{1}\right)}+\left\|\partial_{t} v\right\|_{H^{1.5}} \int_{0}^{t} P$. Adding $\left\|\partial_{t} v^{3}\right\|_{L^{2}\left(\Gamma_{1}\right)}$ to both sides then gives

$$
\begin{aligned}
\left\|\partial_{t} v^{3}\right\|_{H^{1}\left(\Gamma_{1}\right)} & \lesssim\left\|\Pi \bar{\partial} \partial_{t} v\right\|_{L^{2}\left(\Gamma_{1}\right)}+\left\|\partial_{t} v\right\|_{H^{0.5+\delta_{0}}}+\left\|\partial_{t} v\right\|_{H^{1.5}} \int_{0}^{t} P \\
& \lesssim\left\|\Pi \bar{\partial} \partial_{t} v\right\|_{L^{2}\left(\Gamma_{1}\right)}+\left\|\partial_{t} v\right\|_{L^{2}}+\epsilon_{0}\left\|\partial_{t} v\right\|_{H^{1.5}}+\left\|\partial_{t} v\right\|_{H^{1.5}} \int_{0}^{t} P \\
& \lesssim\left\|\Pi \bar{\partial} \partial_{t} v\right\|_{L^{2}\left(\Gamma_{1}\right)}+P_{0}+\int_{0}^{t} P+\epsilon_{0}\left\|\partial_{t} v\right\|_{H^{1.5}}+\left\|\partial_{t} v\right\|_{H^{1.5}} \int_{0}^{t} P
\end{aligned}
$$

where we used interpolation and Young's inequalities in the second step. We may rewrite the resulting inequality as

$$
\left\|\partial_{t} v^{3}\right\|_{H^{1}\left(\Gamma_{1}\right)} \lesssim\left\|\Pi \bar{\partial} \partial_{t} v\right\|_{L^{2}\left(\Gamma_{1}\right)}+\epsilon_{0}\left\|\partial_{t} v\right\|_{H^{1.5}}+P_{0}+\left(1+\left\|\partial_{t} v\right\|_{H^{1.5}}\right) \int_{0}^{t} P .
$$

Next, we apply (10.1) with $X=\bar{\partial} \tilde{\partial}^{1-\nu / 2} v$, leading to $\bar{\partial} \tilde{\partial}^{1-\nu / 2} v^{3}=\left(\Pi \bar{\partial} \tilde{\partial}^{1-\nu / 2} v\right)^{3}+g^{k l} \partial_{k} \eta_{\alpha} \bar{\partial} \tilde{\partial}^{1-\nu / 2} v^{\alpha} \int_{0}^{t} \partial_{l} v^{3}$. Then $\left\|\bar{\partial} \tilde{\partial}^{1-\nu / 2} v^{3}\right\|_{L^{2}\left(\Gamma_{1}\right)} \lesssim\left\|\Pi \bar{\partial} \tilde{\partial}^{1-\nu / 2} v\right\|_{L^{2}\left(\Gamma_{1}\right)}+\|v\|_{H^{2.5-\nu / 2}} \int_{0}^{t}\|v\|_{H^{2.5+\delta_{0}}}$. Note that the first term on the right side is estimated in Section 7. Adding $\left\|v^{3}\right\|_{L^{2}\left(\Gamma_{1}\right)}$ to both sides gives

$$
\begin{aligned}
\left\|v^{3}\right\|_{H^{2-\nu / 2}\left(\Gamma_{1}\right)} & \lesssim\left\|\Pi \bar{\partial} \tilde{\partial}^{1-\nu / 2} v\right\|_{L^{2}\left(\Gamma_{1}\right)}+\|v\|_{H^{1}}+\|v\|_{H^{2.5-\nu / 2}} \int_{0}^{t} P \\
& \lesssim\left\|\Pi \bar{\partial} \tilde{\partial}^{1-\nu / 2} v\right\|_{L^{2}\left(\Gamma_{1}\right)}+\epsilon_{0}\|v\|_{H^{2.5-\nu / 2}}+\|v\|_{L^{2}}+\|v\|_{H^{2.5-\nu / 2}} \int_{0}^{t} P .
\end{aligned}
$$

We rewrite this as

$$
\left\|v^{3}\right\|_{H^{2-\nu / 2}\left(\Gamma_{1}\right)} \lesssim\left\|\Pi \bar{\partial} \tilde{\partial}^{1-\nu / 2} v\right\|_{L^{2}\left(\Gamma_{1}\right)}+\epsilon_{0}\|v\|_{H^{2.5-\nu / 2}}+P_{0}+\left(\|v\|_{H^{2.5-\nu / 2}}+1\right) \int_{0}^{t} P .
$$


Combining (9.1) with (10.3) and choosing $\epsilon_{0}$ sufficiently small, we get

$$
\|v\|_{H^{2.5-\nu / 2}} \lesssim\left\|\Pi \bar{\partial} \tilde{\partial}^{1-\nu / 2} v\right\|_{L^{2}\left(\Gamma_{1}\right)}+P_{0}+\left(\|v\|_{H^{2.5-\nu / 2}}+1\right) \int_{0}^{t} P .
$$

Combining (9.3) and (10.2) with $\epsilon_{0}>0$ sufficiently small, we obtain

$$
\begin{gathered}
\left\|\partial_{t} v\right\|_{H^{1.5}} \lesssim\left\|\Pi \bar{\partial} \partial_{t} v\right\|_{L^{2}\left(\Gamma_{1}\right)}+\|v\|_{H^{2}}^{2}+P_{0}+\left(1+\left\|\partial_{t} v\right\|_{H^{1.5}}\right) \int_{0}^{t} P \\
\lesssim\left\|\Pi \bar{\partial} \partial_{t} v\right\|_{L^{2}\left(\Gamma_{1}\right)}+\|v\|_{H^{1.5}}^{2(1-\nu) /(2-\nu)}\|v\|_{H^{2.5-\nu / 2}}^{2 /(2-\nu)} \\
\quad+P_{0}+\left(1+\left\|\partial_{t} v\right\|_{H^{1.5}}\right) \int_{0}^{t} P .
\end{gathered}
$$

\section{THE CONCLUDING ESTIMATES}

Now, we are ready to combine all the available inequalities to prove Theorem 2.1.

Proof of Theorem 2.1. Squaring (10.4) and using (7.3), we get

$$
E_{0}^{2} \lesssim P_{0}+\left(E_{0}^{2}+1\right) \int_{0}^{t} P
$$

Also, combining (8.3) and (10.5), we get

$$
E_{1} \lesssim \epsilon_{0} E+E \int_{0}^{t} P+\left(P_{0}+\int_{0}^{t} P\right)\left(G^{3\left(1+2 \delta_{0}\right) / 2(3-\nu)}+H^{3 / 4}+E^{1 /(2-\nu)}+1\right)
$$

from where, using Young's inequality,

$$
E_{1} \lesssim \epsilon_{0} E+E \int_{0}^{t} P+\left(P_{0}+\int_{0}^{t} P\right)\left(G^{3\left(1+2 \delta_{0}\right) / 2(3-\nu)}+H^{3 / 4}+1\right)
$$

Finally, we add (11.1) and (11.2) and choose $\epsilon_{0}$ sufficiently small so we can absorb $2 \epsilon_{0} E$, obtaining

$$
E \lesssim E \int_{0}^{t} P+\left(P_{0}+\int_{0}^{t} P\right)\left(G^{3\left(1+2 \delta_{0}\right) / 2(3-\nu)}+H^{3 / 4}+1\right)
$$

Now, we turn to establishing the control of $\|v\|_{H^{3-\nu}}$. From [35], recall the identity

$$
\begin{aligned}
& \sqrt{g} g^{i j} \partial_{i j}^{2} v^{3}-\sqrt{g} g^{i j} \Gamma_{i j}^{k} \partial_{k} v^{3} \\
& \quad=-\partial_{t}\left(\sqrt{g} g^{i j}\right) \partial_{i j}^{2} \eta^{3}-\partial_{t}\left(\sqrt{g} g^{i j} \Gamma_{i j}^{k}\right) \partial_{k} \eta^{3}-\partial_{t} a^{\mu 3} N_{\mu} q-a^{\mu 3} N_{\mu} \partial_{t} q \text { on } \Gamma_{1},
\end{aligned}
$$

which follows from differentiating (2.6) in $t$ and setting $\alpha=3$. We rewrite the equation above as

$$
\begin{array}{r}
\Delta v^{3}=\left(\delta^{i j}-\sqrt{g} g^{i j}\right) \partial_{i j}^{2} v^{3}+\sqrt{g} g^{i j} \Gamma_{i j}^{k} \partial_{k} v^{3}-\partial_{t}\left(\sqrt{g} g^{i j}\right) \partial_{i j}^{2} \eta^{3} \\
-\partial_{t}\left(\sqrt{g} g^{i j} \Gamma_{i j}^{k}\right) \partial_{k} \eta^{3}-\partial_{t} a^{\mu 3} N_{\mu} q-a^{\mu 3} N_{\mu} \partial_{t} q \text { on } \Gamma_{1}
\end{array}
$$

from where, by ellipticity,

$$
\begin{aligned}
\left\|v^{3}\right\|_{H^{2.5-\nu}\left(\Gamma_{1}\right)} \lesssim \| & \left(\delta^{i j}-\sqrt{g} g^{i j}\right) \partial_{i j}^{2} v^{3}\left\|_{H^{0.5-\nu}\left(\Gamma_{1}\right)}+\right\| \sqrt{g} g^{i j} \Gamma_{i j}^{k} \partial_{k} v^{3} \|_{H^{0.5-\nu}\left(\Gamma_{1}\right)} \\
& +\left\|\partial_{t}\left(\sqrt{g} g^{i j}\right) \partial_{i j}^{2} \eta^{3}\right\|_{H^{0.5-\nu}\left(\Gamma_{1}\right)}+\left\|\partial_{t}\left(\sqrt{g} g^{i j} \Gamma_{i j}^{k}\right) \partial_{k} \eta^{3}\right\|_{H^{0.5-\nu}\left(\Gamma_{1}\right)} \\
& +\left\|\partial_{t} a^{\mu 3} N_{\mu} q\right\|_{H^{0.5-\nu}\left(\Gamma_{1}\right)}+\left\|a^{\mu 3} N_{\mu} \partial_{t} q\right\|_{H^{0.5-\nu}\left(\Gamma_{1}\right)} .
\end{aligned}
$$


Using (6.6), we get

$$
\begin{aligned}
\left\|v^{3}\right\|_{H^{2.5-\nu}\left(\Gamma_{1}\right)} \lesssim & \sum_{i, j}\left(\left\|\delta^{i j}-\sqrt{g} g^{i j}\right\|_{L^{\infty}}+\left\|\delta^{i j}-\sqrt{g} g^{i j}\right\|_{H^{1}\left(\Gamma_{1}\right)}\right)\left\|v^{3}\right\|_{H^{2.5-\nu}\left(\Gamma_{1}\right)} \\
& +\left\|\sqrt{g} g^{i j} \Gamma_{i j}^{k}\right\|_{H^{0.5}\left(\Gamma_{1}\right)}\left\|\partial_{k} v^{3}\right\|_{H^{1-\nu}\left(\Gamma_{1}\right)} \\
& +\left(\left\|\partial_{t}\left(\sqrt{g} g^{i j}\right)\right\|_{L^{\infty}}+\left\|\partial_{t}\left(\sqrt{g} g^{i j}\right)\right\|_{H^{1}\left(\Gamma_{1}\right)}\right)\left\|\partial_{i j}^{2} \eta^{3}\right\|_{H^{0.5-\nu}\left(\Gamma_{1}\right)} \\
& +\left\|\partial_{t}\left(\sqrt{g} g^{i j} \Gamma_{i j}^{k}\right)\right\|_{H^{0.5-\nu}\left(\Gamma_{1}\right)}\left(\left\|\partial_{k} \eta^{3}\right\|_{L^{\infty}}+\left\|\partial_{k} \eta^{3}\right\|_{H^{1}\left(\Gamma_{1}\right)}\right) \\
& +\left\|\partial_{t} a^{\mu 3} N_{\mu}\right\|_{H^{1-\nu}\left(\Gamma_{1}\right)}\|q\|_{H^{0.5}\left(\Gamma_{1}\right)}+\left\|a^{\mu 3} N_{\mu}\right\|_{H^{1-\nu}\left(\Gamma_{1}\right)}\left\|\partial_{t} q\right\|_{H^{0.5}\left(\Gamma_{1}\right)} .
\end{aligned}
$$

Since $\left\|\delta^{i j}-\sqrt{g} g^{i j}\right\|_{L^{\infty}}+\left\|\delta^{i j}-\sqrt{g} g^{i j}\right\|_{H^{1}} \leq \epsilon_{0}$ by Lemma 3.1(iv) (ensuring that $T \leq 1 / C M \epsilon_{0}$ ), and using (recall that $\eta(0)$ is the identity) $\left\|\partial_{k} \eta^{3}\right\|_{L^{\infty}}+\left\|\partial_{k} \eta^{3}\right\|_{H^{1}\left(\Gamma_{1}\right)} \leq \epsilon_{0}$, also by Lemma 3.1(iv), we get

$$
\begin{aligned}
\left\|v^{3}\right\|_{H^{2.5-\nu}\left(\Gamma_{1}\right)} & \lesssim \epsilon_{0}\left\|v^{3}\right\|_{H^{3-\nu}}+\|q\|_{H^{1}}+\left\|\partial_{t} q\right\|_{H^{1}}+\|v\|_{H^{2.5+\delta_{0}}}+P_{0}+\int_{0}^{t} P \\
& \lesssim P_{0}+\left\|\partial_{t} q\right\|_{H^{1}}+\epsilon_{0}\|v\|_{H^{3-\nu}}+\int_{0}^{t} P .
\end{aligned}
$$

(Note that $\|q\|_{H^{1}} \lesssim P_{0}+\int_{0}^{t} P$ and $\|v\|_{H^{2.5+\delta_{0}}} \lesssim \epsilon_{0}\|v\|_{H^{3-\nu}}+P_{0}+\int_{0}^{t} P$.)

Combining (11.4) with (9.2) and setting $\epsilon_{0}>0$ sufficiently small, we obtain

$$
F=\|v\|_{H^{3-\nu}} \lesssim P_{0}+\left\|\partial_{t} q\right\|_{H^{1}}+\int_{0}^{t} P \lesssim H+P_{0}+\int_{0}^{t} P,
$$

while by (11.3) we have

$$
E \lesssim E \int_{0}^{t} P+\left(P_{0}+\int_{0}^{t} P\right)\left(G^{3\left(1+2 \delta_{0}\right) / 2(3-\nu)}+H^{3 / 4}+1\right) .
$$

Also, (4.3) reads

$$
G \lesssim\left(P_{0}+\int_{0}^{t} P\right) E^{(3-\nu) / 3}
$$

Substituting (11.7) in (11.6) and using Young's inequality then yields

$$
E \lesssim E \int_{0}^{t} P+\left(P_{0}+\int_{0}^{t} P\right)\left(H^{3 / 4}+1\right)
$$

Next, we have (4.4), which is

$$
H \lesssim E+\left(P_{0}+\int_{0}^{t} P\right)\left(E^{(7+2 \nu) / 6}+E^{1 / 2} G^{\left(1+\nu+2 \delta_{0}\right) /(3-\nu)}+F^{\nu /(1-\nu)} E^{(1-2 \nu) /(2-2 \nu)}\right) .
$$

The inequality (11.7) then gives

$$
\begin{aligned}
H & \lesssim E+\left(P_{0}+\int_{0}^{t} P\right)\left(E^{(7+2 \nu) / 6}+E^{\left(5+2 \nu+4 \delta_{0}\right) / 6}+F^{\nu /(1-\nu)} E^{(1-2 \nu) /(2-2 \nu)}\right) \\
& \lesssim E+\left(P_{0}+\int_{0}^{t} P\right)\left(E^{(7+2 \nu) / 6}+F^{\nu /(1-\nu)} E^{(1-2 \nu) /(2-2 \nu)}\right)
\end{aligned}
$$

where we used Young's inequality and $\left(5+2 \nu+4 \delta_{0}\right) / 6<1$ in the last step. Replacing (11.5) into (11.9), we get

$$
H \lesssim E+\left(P_{0}+\int_{0}^{t} P\right)\left(E^{(7+2 \nu) / 6}+H^{\nu /(1-\nu)} E^{(1-2 \nu) /(2-2 \nu)}\right)
$$

from where, using Young's inequality to absorb $H^{\nu /(1-\nu)}$ into the left side (note that $\nu /(1-\nu)<1$ by the restriction on $\nu$ ), we get

$$
H \lesssim E+\left(P_{0}+\int_{0}^{t} P\right)\left(E^{(7+2 \nu) / 6}+E^{1 / 2}\right) \lesssim E+\left(P_{0}+\int_{0}^{t} P\right) E^{(7+2 \nu) / 6} .
$$


We need to combine this inequality with (11.8). Observe that

$$
\frac{7+2 \nu}{6} \frac{3}{4}<1
$$

which follows from $0 \leq \nu<1 / 2$. Thus, we may choose $\tilde{\epsilon}>0$ such that

$$
\frac{7+2 \nu}{6} \frac{3}{4}(1+\tilde{\epsilon})<1
$$

Then replacing (11.10) in (11.8), we get

$$
E \lesssim E \int_{0}^{t} P+\left(P_{0}+\int_{0}^{t} P\right)\left(E^{3 / 4}+E^{3(7+2 \nu) / 24}\right) \lesssim E \int_{0}^{t} P+\left(P_{0}+\int_{0}^{t} P\right)\left(E^{3 / 4}+E^{1 /(1+\tilde{\epsilon})}\right)
$$

where we used (11.12) in the last step. Using Young's inequality on (11.13), we get

$$
E \lesssim P_{0}+E \int_{0}^{t} P
$$

Note that $P$ here and below depends on $E, F, G$, and $H$, i.e., $P=P(E, F, G, H)$. The inequality (11.14) is combined with (11.10), i.e.,

$$
H \lesssim\left(P_{0}+\int_{0}^{t} P\right) E^{(7+2 \nu) / 6}
$$

In addition, we have an inequality for $F$, which is (11.5) with (11.15) applied to it,

$$
F \lesssim\left(P_{0}+\int_{0}^{t} P\right) E^{(7+2 \nu) / 6}
$$

Finally, by (11.7), we have

$$
G \lesssim\left(P_{0}+\int_{0}^{t} P\right) E^{(3-\nu) / 3}
$$

A barrier technique applied to (11.14)-(11.17) then leads to the boundedness of $E, F, G$, and $H$ for a sufficiently small $T>0$ and the proof is concluded.

\section{The CASE OF A General DOMAIN}

In this section, we show how to adapt the ideas used to prove Theorem 2.1, where the initial surface was flat, to the case of a general bounded domain. The physical situation which we have in mind is that of a water droplet with surface tension. In this case the fluid domain does not have a rigid bottom, and thus only equations (2.2)-(2.6) are considered. Note however that the presence of a rigid bottom can also be handled with minor modifications. If $U$ is a domain in $\mathbb{R}^{3},\|\partial U\|_{s}$ is the $H^{s}$ norm of the boundary of the domain, defined in the usual way via local representations as graphs.

Theorem 12.1. Let $\sigma>0$ and $\epsilon \in[0,1 / 2)$. Assume that $v_{0}$ is a smooth divergence-free vector field on a bounded domain $\Omega \subset \mathbb{R}^{3}$ with smooth boundary $\Gamma$, and denote by $N$ the unit outer normal to $\Gamma$. Then there exist $C_{*}>0$ and $T_{*}>0$, depending only on $\left\|v_{0}\right\|_{H^{2.5+\epsilon}},\left\|v_{0} \cdot N\right\|_{H^{2.5}(\Gamma)}, \sigma>0$, and $\|\Gamma\|_{H^{3.75+\epsilon / 2}}$, such that any smooth solution $(v, q)$ to (2.2)-(2.6) with the initial condition $v_{0}$ and defined on the time interval $\left[0, T_{*}\right]$ satisfies

$$
\|v\|_{H^{2.5+\epsilon}}+\left\|\partial_{t} v\right\|_{H^{1.5}}+\left\|\partial_{t}^{2} v\right\|_{L^{2}}+\|q\|_{H^{2.25+\epsilon / 2}}+\left\|\partial_{t} q\right\|_{H^{1}} \leq C_{*} .
$$

Moreover, $\|\Gamma(t)\|_{H^{3+\epsilon}} \leq C_{*}$ for $t \in\left[0, T_{*}\right]$, where $\Gamma(t)=\eta(t)(\Gamma)$. 
As in Theorem 2.1, the dependence of $C_{*}$ and $T_{*}$ on $\left\|v_{0} \cdot N\right\|_{H^{2.5}(\Gamma)}$ occurs to guarantee that $\partial_{t}^{2} v$ belongs to $L^{2}$ at time zero. More precisely, solving for $\partial_{t}^{2} v(0)$ in terms of $v(0)$ and $q(0)$ as in Remark 4.3, we can bound $\partial_{t}^{2} v(0)$ in $L^{2}$ in terms of the initial data if $v_{0} \cdot N \in H^{2.5}(\Gamma)$. However, instead of solving for time-differentiated quantities in terms of the initial data to determine regularity conditions on the latter, many times it is preferable to directly state the a priori estimate upon the assumption that the energy we seek to bound is finite at time zero, as done for example in [25]. Therefore, introducing

$$
N(t)=\|v(t)\|_{H^{2.5+\epsilon}}+\left\|\partial_{t} v(t)\right\|_{H^{1.5}}+\left\|\partial_{t}^{2} v(t)\right\|_{L^{2}}+\|q(t)\|_{H^{2.25+\epsilon / 2}}+\left\|\partial_{t} q(t)\right\|_{H^{1}},
$$

we have the following.

Theorem 12.2. Let $\sigma>0$ and $\epsilon \in[0,1 / 2)$. Assume that $v_{0}$ is a smooth divergence-free vector field on a bounded domain $\Omega \subset \mathbb{R}^{3}$ with smooth boundary $\Gamma$. Then there exist $C_{*}>0$ and $T_{*}>0$ depending only on $N(0), \sigma>0$, such that any smooth solution $(v, q)$ to (2.2)-(2.6) with the initial condition $v_{0}$ and defined on the time interval $\left[0, T_{*}\right]$ satisfies $N(t) \leq C_{*}$.

We remark that Theorem 12.1 entails some derivative loss for the boundary, i.e., a $H^{3.75+\epsilon / 2}$ initial boundary $\Gamma$ yields only a $H^{3+\epsilon}$ moving boundary $\Gamma(t)$. This loss of regularity is known to be prevented in $H^{s}$ for $s \geq 4$ $[25,70]$. It seems challenging, however, to avoid some loss of derivatives for the boundary evolution when working in such low regularity spaces as presented here. It should be stressed, however, that some regularity of the boundary is propagated, namely, $\Gamma(t)$ is in $H^{3+\epsilon}$, thus more regular than the flow $\left.\eta\right|_{\Gamma}$ which is guaranteed to be only in $H^{2+\epsilon}(\Gamma)$.

We now turn to the proof of Theorems 12.1 and 12.2. The crucial observation is that in appropriate coordinates that flatten the boundary near a point, the equations take exactly the same form as (2.2)-(2.6), with $\partial_{i}$, for $i=1,2$, being tangent to the boundary, as in the case of the domain (2.1).

More precisely, given $y_{0} \in \partial \Omega$, we take coordinates that flatten the boundary near $y_{0}$. This means that there exist $r, R>0$ and a diffeomorphism $\Psi: B_{R}(0,0,1) \cap\left\{x^{3} \leq 1\right\} \rightarrow B_{r}\left(y_{0}\right) \cap \Omega$ such that (after a rigid motion and relabeling the coordinates if necessary) we have $\Psi\left(x^{1}, x^{2}, x^{3}\right)=\left(x^{1}, x^{2}, x^{3}+\psi\left(x^{1}, x^{2}\right)\right)$, where $\psi: B_{R}(0) \cap\left\{x^{3}=\right.$ $1\} \rightarrow \mathbb{R}$ is a smooth function. Note that $\operatorname{det} D \Psi=\operatorname{det} D \Psi^{-1}=1$. Consider the Lagrangian map $\eta: \Omega \rightarrow \Omega(t)$, and set $\tilde{\eta}=\eta \circ \Psi$, which is defined in the domain of $\Psi$. Then $\partial_{t} \tilde{\eta}=\partial_{t} \eta \circ \Psi=u \circ \eta \circ \Psi=u \circ \tilde{\eta}$, where $u$ is the Eulerian velocity, i.e., the velocity in the moving domain $\Omega(t)$. It follows that if we introduce $\tilde{v}=u \circ \tilde{\eta}$ and $\tilde{q}=p \circ \tilde{\eta}$, where $p$ is the Eulerian pressure, then $\tilde{v}$ and $\tilde{q}$ satisfy equations (2.2)-(2.6) with all variables replaced by their respective $\sim$ counter-parts - except that these equations are now defined only locally, i.e., in $B_{R}(0) \cap\left\{x^{3} \leq 1\right\}$. We thus use suitably chosen cut-off functions to produce local estimates, passing to a global estimate by a simple addition procedure. In order to simplify the exposition, we will omit tildes from all quantities and continue to label $\eta, v$, and $q$, which are only locally defined, the Lagrangian map, velocity, and pressure, respectively.

We need expressions for $\eta(0), a(0)$, and $g_{i j}(0)$, which now are slightly more complicated than in the case of the domain (2.1). We have

$$
\begin{aligned}
& \eta(0, x)=\left(x^{1}, x^{2}, x^{3}+\psi\left(x^{1}, x^{2}\right)\right), \partial_{i} \eta^{\mu}(0)=\delta_{i}^{\mu}+\delta^{\mu 3} \partial_{i} \psi, g_{i j}(0)=\delta_{i j}+\partial_{i} \psi \partial_{j} \psi, \\
& \text { and } g(0)=1+\left(\partial_{1} \psi\right)^{2}+\left(\partial_{2} \psi\right)^{2}
\end{aligned}
$$

where we recall that $g$ is the determinant of $\left(g_{i j}\right)$. Also,

$$
g^{-1}(0)=\frac{1}{1+\left(\partial_{1} \psi\right)^{2}+\left(\partial_{2} \psi\right)^{2}}\left[\begin{array}{cc}
1+\left(\partial_{2} \psi\right)^{2} & -\partial_{1} \psi \partial_{2} \psi \\
-\partial_{1} \psi \partial_{2} \psi & 1+\left(\partial_{1} \psi\right)^{2}
\end{array}\right], a(0)=\left[\begin{array}{ccc}
1 & 0 & 0 \\
0 & 1 & 0 \\
-\partial_{1} \psi & -\partial_{2} \psi & 1
\end{array}\right] .
$$

In the proof of Theorem 2.1, for which $\psi \equiv 0$, we used the above quantities at time zero to produce some small parameters in the energy estimates. In order to apply the same argument here, we need $\nabla \psi$ to be small. This can be achieved as follows. Without loss of generality we may assume that $\nabla \psi(0,0,1)=0$. Reducing $R$ and invoking the mean value theorem, we may make $\|\nabla \psi\|_{L^{\infty}(\Gamma)}$ as small as we wish provided that $\psi$ is bounded in $H^{2+\delta}$, where $\delta>0$, which is consistent with Theorem 12.1. Note that the compactness of $\Gamma$ assures that we may take $R \geq R_{0}$ for some fixed $R_{0}$. 
We shall derive estimates near the point $(0,0,1)$, with the variables defined in the ball of radius $R / 2$, where $R>0$ is as introduced above in the construction of the local parameterization of $\Omega$. Let $\theta$ be a smooth cut-off function such that $0 \leq \theta \leq 1$ with $\theta \equiv 1$ on $\bar{B}_{R / 5}(0,0,1)$ and $\operatorname{supp} \theta \subseteq B_{R / 4}(0,0,1)$. In what follows, all integrands carry a cut-off function of this type. Therefore, extending all quantities to be identically zero outside $B_{R / 4}(0,0,1)$, we may consider the equations and variables defined on the domain $\tilde{\Omega}=\mathbb{T}^{2} \times[0,1]$. This will make it easier to adapt the estimates from Section 7. Also, as in that section, we shall denote the upper boundary of $\tilde{\Omega}$ by $\Gamma_{1}$ and the lower boundary by $\Gamma_{0}$. However, unlike Section 7, no integral over $\Gamma_{0}$ is present since all variables vanish there in view of the way they have been extended.

We now apply the energy estimates of Section 7 with ,

$$
\mathcal{E}=\tilde{\partial}^{1-\nu / 2}(\theta \cdot)
$$

obtaining

$$
\begin{aligned}
\frac{1}{2} \frac{d}{d t}\left\|\mathcal{E} \partial_{t} v\right\|_{L^{2}}^{2}= & -\int_{\tilde{\Omega}} \mathcal{E} \partial_{t}\left(a^{\mu \alpha} \partial_{\mu} q\right) \mathcal{E} \partial_{t} v_{\alpha}=-\int_{\tilde{\Omega}} \tilde{\partial}^{1-\nu / 2}\left(\theta \partial_{t}\left(a^{\mu \alpha} \partial_{\mu} q\right)\right) \tilde{\partial}^{1-\nu / 2}\left(\theta \partial_{t} v_{\alpha}\right) \\
= & -\int_{\tilde{\Omega}} \theta \partial_{t}\left(a^{\mu \alpha} \partial_{\mu} q\right) \tilde{\partial}^{2-\nu}\left(\theta \partial_{t} v_{\alpha}\right) \\
= & \int_{\tilde{\Omega}} \theta \partial_{t}\left(a^{\mu \alpha} q\right) \tilde{\partial}^{2-\nu}\left(\theta \partial_{t} \partial_{\mu} v_{\alpha}\right)-\int_{\Gamma_{1}} \theta \partial_{t}\left(N_{\mu} a^{\mu \alpha} q\right) \tilde{\partial}^{2-\nu}\left(\theta \partial_{t} v_{\alpha}\right) \\
& \quad+\int_{\tilde{\Omega}} \partial_{\mu} \theta \partial_{t}\left(a^{\mu \alpha} q\right) \tilde{\partial}^{2-\nu}\left(\theta \partial_{t} v_{\alpha}\right)+\int_{\tilde{\Omega}} \theta \partial_{t}\left(a^{\mu \alpha} q\right) \tilde{\partial}^{2-\nu}\left(\partial_{\mu} \theta \partial_{t} v_{\alpha}\right),
\end{aligned}
$$

from where

$$
\begin{aligned}
\frac{1}{2} \frac{d}{d t}\left\|\mathcal{E} \partial_{t} v\right\|_{L^{2}}^{2}= & \int_{\tilde{\Omega}} \mathcal{E} \partial_{t}\left(a^{\mu \alpha} q\right) \mathcal{E} \partial_{t} \partial_{\mu} v_{\alpha}-\int_{\Gamma_{1}} \mathcal{E} \partial_{t}\left(N_{\mu} a^{\mu \alpha} q\right) \mathcal{E} \partial_{t} v_{\alpha} \\
& +\int_{\tilde{\Omega}} \tilde{\partial}^{0.5-\nu}\left(\partial_{\mu} \theta \partial_{t}\left(a^{\mu \alpha} q\right)\right) \tilde{\partial}^{1.5}\left(\theta \partial_{t} v_{\alpha}\right)+\int_{\tilde{\Omega}} \tilde{\partial}^{0.5-\nu}\left(\theta \partial_{t}\left(a^{\mu \alpha} q\right)\right) \tilde{\partial}^{1.5}\left(\partial_{\mu} \theta \partial_{t} v_{\alpha}\right) .
\end{aligned}
$$

By (12.3), we have $\frac{1}{2} \frac{d}{d t}\left\|\tilde{\partial}^{1-\nu / 2}\left(\theta \partial_{t} v\right)\right\|_{L^{2}}^{2}=I_{1}+I_{2}+I_{3}+I_{4}+I_{5}$, where

$$
\begin{aligned}
I_{1} & =\int_{\tilde{\Omega}} \tilde{\partial}^{1-\nu / 2}\left(\theta \partial_{t} a^{\mu \alpha} q\right) \tilde{\partial}^{1-\nu / 2}\left(\theta \partial_{t} \partial_{\mu} v_{\alpha}\right) \\
I_{2} & =\int_{\tilde{\Omega}} a^{\mu \alpha} \tilde{\partial}^{2-\nu}\left(\theta \partial_{t} q\right) \theta \partial_{t} \partial_{\mu} v_{\alpha} \\
I_{3} & =\int_{\tilde{\Omega}}\left(\tilde{\partial}^{2-\nu}\left(\theta a^{\mu \alpha} \partial_{t} q\right)-a^{\mu \alpha} \tilde{\partial}^{2-\nu}\left(\theta \partial_{t} q\right)\right) \theta \partial_{t} \partial_{\mu} v_{\alpha} \\
I_{4} & =-\int_{\Gamma_{1}} \tilde{\partial}^{1-\nu / 2}\left(\theta \partial_{t}\left(N_{\mu} a^{\mu \alpha} q\right)\right) \tilde{\partial}^{1-\nu / 2}\left(\theta \partial_{t} v_{\alpha}\right) \\
I_{5} & =\int_{\tilde{\Omega}} \tilde{\partial}^{0.5-\nu}\left(\partial_{\mu} \theta \partial_{t}\left(a^{\mu \alpha} q\right)\right) \tilde{\partial}^{1.5}\left(\theta \partial_{t} v_{\alpha}\right)+\int_{\tilde{\Omega}} \tilde{\partial}^{0.5-\nu}\left(\theta \partial_{t}\left(a^{\mu \alpha} q\right)\right) \tilde{\partial}^{1.5}\left(\partial_{\mu} \theta \partial_{t} v_{\alpha}\right) .
\end{aligned}
$$

The first term is rewritten as

$$
I_{1}=\int_{\tilde{\Omega}} \tilde{\partial}^{1.5-\nu}\left(\theta \partial_{t} a^{\mu \alpha} q\right) \tilde{\partial}^{0.5}\left(\theta \partial_{t} \partial_{\mu} v_{\alpha}\right) \lesssim\left\|\tilde{\partial}^{1.5-\nu}\left(\theta \partial_{t} a q\right)\right\|_{L^{2}}\left\|\theta \partial_{t} \nabla v\right\|_{H^{0.5}}
$$

Now, let $\bar{\theta}$ be a smooth cut-off function such that $0 \leq \bar{\theta} \leq 1$ with $\operatorname{supp} \bar{\theta} \subseteq B_{R / 3}(0,0,1)$ and $\bar{\theta} \equiv 1$ on $\operatorname{supp} \theta$. We need this cut-off function for an application of the fractional product rule below, as each separate term needs to be properly cut-off. Having $\bar{\theta} \equiv 1$ on $\operatorname{supp} \theta$ assures that we may introduce $\bar{\theta}$ without altering given expressions. We have

$$
\left\|\tilde{\partial}^{1.5-\nu}\left(\theta \partial_{t} a q\right)\right\|_{L^{2}}=\left\|\tilde{\partial}^{1.5-\nu}\left(\theta \partial_{t} a \bar{\theta} q\right)\right\|_{L^{2}} \lesssim\left\|\theta \partial_{t} a\right\|_{H^{2-\nu}}\|\bar{\theta} q\|_{H^{1}}+\left\|\theta \partial_{t} a\right\|_{H^{1}}\|\bar{\theta} q\|_{H^{2-\nu}}
$$


where we used the fractional product rule. Also,

$$
\begin{aligned}
\left\|\theta \partial_{t} \nabla v\right\|_{H^{0.5}} \lesssim\left\|\nabla\left(\theta \partial_{t} v\right)\right\|_{H^{0.5}}+\left\|\nabla \theta \partial_{t} v\right\|_{H^{0.5}} \lesssim\left\|\bar{\theta} \theta \partial_{t} v\right\|_{H^{1.5}}+\left\|\bar{\theta} \nabla \theta \partial_{t} v\right\|_{H^{0.5}} \\
\quad \lesssim\|\theta\|_{H^{1.5+\delta_{0}}}\left\|\bar{\theta} \partial_{t} v\right\|_{H^{1.5}}+\|\nabla \theta\|_{H^{1.5+\delta_{0}}}\left\|\bar{\theta} \partial_{t} v\right\|_{H^{0.5}} \lesssim\left\|\bar{\theta} \partial_{t} v\right\|_{H^{1.5}}
\end{aligned}
$$

Therefore, we get $I_{1} \lesssim\left\|\theta \partial_{t} a\right\|_{H^{2-\nu}}\|\bar{\theta} q\|_{H^{1}}\left\|\bar{\theta} \partial_{t} v\right\|_{H^{1.5}}+\left\|\theta \partial_{t} a\right\|_{H^{1}}\|\bar{\theta} q\|_{H^{2-\nu}}\left\|\bar{\theta} \partial_{t} v\right\|_{H^{1.5}}$. Next, by the divergencefree condition (2.3) we have

$$
\begin{aligned}
I_{2} & =-\int_{\tilde{\Omega}} \partial_{t} a^{\mu \alpha} \tilde{\partial}^{2-\nu}\left(\theta \partial_{t} q\right) \theta \partial_{\mu} v_{\alpha}=-\int_{\tilde{\Omega}} \tilde{\partial}^{1-\nu}\left(\partial_{t} a^{\mu \alpha} \theta \partial_{\mu} v_{\alpha}\right) \tilde{\partial}\left(\theta \partial_{t} q\right) \\
& =-\int_{\tilde{\Omega}} \tilde{\partial}^{1-\nu}\left(\bar{\theta} \partial_{t} a^{\mu \alpha} \theta \partial_{\mu} v_{\alpha}\right) \tilde{\partial}\left(\theta \partial_{t} q\right) \lesssim\left\|\tilde{\partial}^{1-\nu}\left(\bar{\theta} \partial_{t} a^{\mu \alpha} \theta \partial_{\mu} v_{\alpha}\right)\right\|_{L^{2}}\left\|\theta \partial_{t} q\right\|_{H^{1}}
\end{aligned}
$$

and thus, using the fractional chain rule, $I_{2} \lesssim\left\|\theta \partial_{t} a^{\mu \alpha}\right\|_{H^{1.5}}\left\|\theta \partial_{\mu} v_{\alpha}\right\|_{H^{1-\nu}}\left\|\theta \partial_{t} q\right\|_{H^{1}}$. For $I_{3}$, we have, as in (7.5),

$$
\begin{aligned}
I_{3} & =\int_{\tilde{\Omega}}\left(\tilde{\partial}^{2-\nu}\left(\bar{\theta} a^{\mu \alpha} \theta \partial_{t} q\right)-\bar{\theta} a^{\mu \alpha} \tilde{\partial}^{2-\nu}\left(\theta \partial_{t} q\right)\right) \theta \partial_{t} \partial_{\mu} v_{\alpha} \\
& \lesssim\left\|\tilde{\partial}^{2-\nu}\left(\bar{\theta} a^{\mu \alpha} \theta \partial_{t} q\right)-\bar{\theta} a^{\mu \alpha} \tilde{\partial}^{2-\nu}\left(\theta \partial_{t} q\right)\right\|_{L^{3 / 2}}\left\|\theta \partial_{t} \partial_{\mu} v\right\|_{L^{3}} \\
& \lesssim\|\bar{\theta} a\|_{H^{2-\nu}}\left\|\theta \partial_{t} q\right\|_{H^{1}}\left\|\theta \partial_{t} \partial_{\mu} v\right\|_{L^{3}} \lesssim\|\bar{\theta} a\|_{H^{2-\nu}}\left\|\theta \partial_{t} q\right\|_{H^{1}}\left\|\bar{\theta} \partial_{t} v\right\|_{H^{1.5}}
\end{aligned}
$$

where we used

$$
\begin{aligned}
\left\|\theta \partial_{t} \partial_{\mu} v\right\|_{L^{3}} & \leq\left\|\partial_{\mu}\left(\theta \partial_{t} v\right)\right\|_{L^{3}}+\left\|\partial_{\mu} \theta \partial_{t} v\right\|_{L^{3}}=\left\|\partial_{\mu}\left(\theta \partial_{t} v\right)\right\|_{L^{3}}+\left\|\bar{\theta} \partial_{\mu} \theta \partial_{t} v\right\|_{L^{3}} \\
& \lesssim\left\|\partial_{\mu}\left(\theta \partial_{t} v\right)\right\|_{H^{0.5}}+\left\|\bar{\theta} \partial_{t} v\right\|_{L^{3}} \lesssim\left\|\theta \partial_{t} v\right\|_{H^{1.5}}+\left\|\bar{\theta} \partial_{t} v\right\|_{H^{0.5}} \lesssim\left\|\bar{\theta} \partial_{t} v\right\|_{H^{1.5}}
\end{aligned}
$$

in the last step.

Before treating the most difficult term $I_{4}$, we bound the lower order term $I_{5}$ which is the sum of two terms, denoted by $I_{51}$ and $I_{52}$. For the first one, we write

$$
\begin{aligned}
& I_{51}=\int_{\tilde{\Omega}} \tilde{\partial}^{0.5-\nu}\left(\partial_{\mu} \theta \partial_{t}\left(a^{\mu \alpha} q\right)\right) \tilde{\partial}^{1.5}\left(\theta \partial_{t} v_{\alpha}\right) \lesssim\left\|\tilde{\partial}^{0.5-\nu}\left(\partial_{\mu} \theta \partial_{t}\left(\bar{\theta} a^{\mu \alpha} \bar{\theta} q\right)\right)\right\|_{L^{2}}\left\|\tilde{\partial}^{1.5}\left(\theta \partial_{t} v_{\alpha}\right)\right\|_{L^{2}} \\
& \vdots\|\theta\|_{H^{2.5+\delta_{0}}}\left\|\bar{\theta} \partial_{t} a\right\|_{H^{1.5-\nu / 2}}\|\bar{\theta} q\|_{H^{0.5-\nu / 2}}\left\|\bar{\theta} \partial_{t} v_{\alpha}\right\|_{H^{1.5}} \\
& \quad+\|\theta\|_{H^{2.5+\delta_{0}}}\|\bar{\theta} a\|_{H^{1.5-\nu / 2}}\left\|\bar{\theta} \partial_{t} q\right\|_{H^{0.5-\nu / 2}}\left\|\bar{\theta} \partial_{t} v_{\alpha}\right\|_{H^{1.5}}
\end{aligned}
$$

while for the second one we have similarly

$$
\begin{aligned}
I_{52} & =\int_{\tilde{\Omega}} \tilde{\partial}^{0.5-\nu}\left(\theta \partial_{t}\left(a^{\mu \alpha} q\right)\right) \tilde{\partial}^{1.5}\left(\partial_{\mu} \theta \partial_{t} v_{\alpha}\right) \lesssim\left\|\tilde{\partial}^{0.5-\nu}\left(\theta \partial_{t}\left(\bar{\theta} a^{\mu \alpha} \bar{\theta} q\right)\right)\right\|_{L^{2}}\left\|\tilde{\partial}^{1.5}\left(\partial_{\mu} \theta \partial_{t} v_{\alpha}\right)\right\|_{L^{2}} \\
& \lesssim\|\theta\|_{H^{1.5+\delta_{0}}}\left\|\bar{\theta} \partial_{t} a\right\|_{H^{1.5-\nu / 2}}\|\bar{\theta} q\|_{H^{0.5-\nu / 2}}\left\|\bar{\theta} \partial_{t} v_{\alpha}\right\|_{H^{1.5}}+\|\theta\|_{H^{1.5+\delta_{0}}}\|\bar{\theta} a\|_{H^{1.5-\nu / 2}}\left\|\bar{\theta} \partial_{t} q\right\|_{H^{0.5-\nu / 2}}\left\|\bar{\theta} \partial_{t} v_{\alpha}\right\|_{H^{1.5}} .
\end{aligned}
$$

Now, we turn to the term $I_{4}$, for which we modify the considerations in Section 6. With $\mathcal{E}$ defined in (12.2), we first obtain the first equality in (6.1), i.e.,

$$
\begin{aligned}
I_{4}= & \int_{\Gamma_{1}} \mathcal{E} \partial_{t} v_{\alpha} \mathcal{E} \partial_{i}\left(\sqrt{g} g^{i j}\left(\delta_{\lambda}^{\alpha}-g^{k l} \partial_{k} \eta^{\alpha} \partial_{l} \eta_{\lambda}\right) \partial_{j} v^{\lambda}+\sqrt{g}\left(g^{i j} g^{k l}-g^{l j} g^{i k}\right) \partial_{j} \eta^{\alpha} \partial_{k} \eta_{\lambda} \partial_{l} v^{\lambda}\right) \\
=- & \int_{\Gamma_{1}} \partial_{i} \mathcal{E} \partial_{t} v_{\alpha} \mathcal{E}\left(\sqrt{g} g^{i j}\left(\delta_{\lambda}^{\alpha}-g^{k l} \partial_{k} \eta^{\alpha} \partial_{l} \eta_{\lambda}\right) \partial_{j} v^{\lambda}\right) \\
& -\int_{\Gamma_{1}} \partial_{i} \mathcal{E} \partial_{t} v_{\alpha} \mathcal{E}\left(\sqrt{g}\left(g^{i j} g^{k l}-g^{l j} g^{i k}\right) \partial_{j} \eta^{\alpha} \partial_{k} \eta_{\lambda} \partial_{l} v^{\lambda}\right) \\
& -\int_{\Gamma_{1}} \mathcal{E} \partial_{t} v_{\alpha} \tilde{\partial}^{1-\nu / 2}\left(\partial_{i} \theta \sqrt{g} g^{i j}\left(\delta_{\lambda}^{\alpha}-g^{k l} \partial_{k} \eta^{\alpha} \partial_{l} \eta_{\lambda}\right) \partial_{j} v^{\lambda}\right) \\
& -\int_{\Gamma_{1}} \mathcal{E} \partial_{t} v_{\alpha} \tilde{\partial}^{1-\nu / 2}\left(\partial_{i} \theta\left(\sqrt{g}\left(g^{i j} g^{k l}-g^{l j} g^{i k}\right) \partial_{j} \eta^{\alpha} \partial_{k} \eta_{\lambda} \partial_{l} v^{\lambda}\right)\right)=I_{41}+I_{42}+I_{43}+I_{44}
\end{aligned}
$$


Using (6.2), we rewrite

$$
\begin{aligned}
I_{41}=- & \int_{\Gamma_{1}} \tilde{\partial}^{1-\nu / 2}\left(\theta \sqrt{g} g^{i j} \Pi_{\lambda}^{\alpha} \partial_{j} v^{\lambda}\right) \partial_{i} \tilde{\partial}^{1-\nu / 2}\left(\theta \partial_{t} v_{\alpha}\right) \\
=- & \int_{\Gamma_{1}} \tilde{\partial}^{1-\nu / 2}\left(\bar{\theta} \sqrt{g} g^{i j} \Pi_{\lambda}^{\alpha} \theta \partial_{j} v^{\lambda}\right) \tilde{\partial}^{1-\nu / 2}\left(\theta \partial_{i} \partial_{t} v_{\alpha}\right) \\
& -\int_{\Gamma_{1}} \tilde{\partial}^{1-\nu / 2}\left(\theta \sqrt{g} g^{i j} \Pi_{\lambda}^{\alpha} \partial_{j} v^{\lambda}\right) \tilde{\partial}^{1-\nu / 2}\left(\partial_{i} \theta \partial_{t} v_{\alpha}\right) \\
=- & \int_{\Gamma_{1}} \bar{\theta} \sqrt{g} g^{i j} \Pi_{\lambda}^{\alpha} \tilde{\partial}^{1-\nu / 2}\left(\theta \partial_{j} v^{\lambda}\right) \tilde{\partial}^{1-\nu / 2}\left(\theta \partial_{i} \partial_{t} v_{\alpha}\right) \\
& -\int_{\Gamma_{1}}\left(\tilde{\partial}^{1-\nu / 2}\left(\bar{\theta} \sqrt{g} g^{i j} \Pi_{\lambda}^{\alpha} \theta \partial_{j} v^{\lambda}\right)-\bar{\theta} \sqrt{g} g^{i j} \Pi_{\lambda}^{\alpha} \tilde{\partial}^{1-\nu / 2}\left(\theta \partial_{j} v^{\lambda}\right)\right) \tilde{\partial}^{1-\nu / 2}\left(\theta \partial_{i} \partial_{t} v_{\alpha}\right) \\
& -\int_{\Gamma_{1}} \tilde{\partial}^{1-\nu / 2}\left(\theta \sqrt{g} g^{i j} \Pi_{\lambda}^{\alpha} \partial_{j} v^{\lambda}\right) \tilde{\partial}^{1-\nu / 2}\left(\partial_{i} \theta \partial_{t} v_{\alpha}\right)=I_{411}+I_{412}+I_{413} .
\end{aligned}
$$

Using $\Pi_{\lambda}^{\alpha}=\Pi_{\mu}^{\alpha} \Pi_{\lambda}^{\mu}$, the first term equals

$$
\begin{aligned}
I_{411} & =-\int_{\Gamma_{1}} \bar{\theta} \sqrt{g} g^{i j} \Pi_{\lambda}^{\mu} \tilde{\partial}^{1-\nu / 2}\left(\theta \partial_{j} v^{\lambda}\right) \Pi_{\mu}^{\alpha} \tilde{\partial}^{1-\nu / 2}\left(\theta \partial_{t} \partial_{i} v_{\alpha}\right) \\
& =-\int_{\Gamma_{1}} \bar{\theta} \sqrt{g} g^{i j} \Pi_{\lambda}^{\mu} \tilde{\partial}^{1-\nu / 2} \partial_{j}\left(\theta v^{\lambda}\right) \Pi_{\mu}^{\alpha} \tilde{\partial}^{1-\nu / 2} \partial_{i}\left(\theta \partial_{t} v_{\alpha}\right)+\text { l.o.t. } \\
& =-\frac{1}{2} \frac{d}{d t} \int_{\Gamma_{1}} \bar{\theta} \sqrt{g} g^{i j} \Pi_{\lambda}^{\mu} \partial_{j} \tilde{\partial}^{1-\nu / 2}\left(\theta v^{\lambda}\right) \Pi_{\mu}^{\alpha} \partial_{i} \tilde{\partial}^{1-\nu / 2}\left(\theta v_{\alpha}\right)+\text { l.o.t. }
\end{aligned}
$$

It is easy to check that $I_{412}$ and $I_{413}$ constitute lower order terms. We thus obtain

$$
\begin{aligned}
I_{41}=- & \frac{1}{2} \frac{d}{d t} \int_{\Gamma_{1}} \bar{\theta} \sqrt{g} g^{i j} \Pi_{\lambda}^{\mu} \partial_{j} \tilde{\partial}^{1-\nu / 2}\left(\theta v^{\lambda}\right) \Pi_{\mu}^{\alpha} \partial_{i} \tilde{\partial}^{1-\nu / 2}\left(\theta v_{\alpha}\right)+\text { l.o.t. } \\
=- & \frac{1}{2} \frac{d}{d t} \int_{\Gamma_{1}} \sqrt{g(0)} g^{i j} \bar{\theta} \Pi_{\lambda}^{\mu} \partial_{j} \tilde{\partial}^{1-\nu / 2}\left(\theta v^{\lambda}\right) \Pi_{\mu}^{\alpha} \partial_{i} \tilde{\partial}^{1-\nu / 2}\left(\theta v_{\alpha}\right) \\
& -\frac{1}{2} \frac{d}{d t} \int_{\Gamma_{1}} \bar{\theta}\left(\sqrt{g} g^{i j}-\sqrt{g(0)} g^{i j}(0)\right) \Pi_{\lambda}^{\mu} \partial_{j} \tilde{\partial}^{1-\nu / 2}\left(\theta v^{\lambda}\right) \Pi_{\mu}^{\alpha} \partial_{i} \tilde{\partial}^{1-\nu / 2}\left(\theta v_{\alpha}\right)+\text { l.o.t. }
\end{aligned}
$$

The first term on the right hand side leads to the needed coercive term, providing the control of the $H^{2-\nu / 2}(\Gamma)$ norm of $\Pi v$. The second term is, after the time integration, dominated by the coercive term by Lemma 3.1(iv). As in Section 6, we have

$$
\begin{aligned}
I_{42}= & -\int_{\Gamma_{1}} \bar{\theta} \sqrt{g}\left(g^{i j} g^{k l}-g^{l j} g^{i k}\right) \partial_{j} \eta^{\alpha} \partial_{k} \eta_{\lambda} \partial_{l} \mathcal{E} v^{\lambda} \partial_{i} \mathcal{E} \partial_{t} v_{\alpha} \\
& -\int_{\Gamma_{1}}\left(\mathcal{E}\left(\bar{\theta} \sqrt{g}\left(g^{i j} g^{k l}-g^{l j} g^{i k}\right) \partial_{j} \eta^{\alpha} \partial_{k} \eta_{\lambda} \partial_{l} v^{\lambda}\right)-\bar{\theta} \sqrt{g}\left(g^{i j} g^{k l}-g^{l j} g^{i k}\right) \partial_{j} \eta^{\alpha} \partial_{k} \eta_{\lambda} \partial_{l} \mathcal{E} v^{\lambda}\right) \partial_{i} \mathcal{E} \partial_{t} v_{\alpha} \\
= & I_{421}+I_{422} .
\end{aligned}
$$

Also, as in Section 6, we have

$$
I_{421}=-\int_{\Gamma_{1}} \frac{\bar{\theta}}{\sqrt{g}}\left(\partial_{t} \operatorname{det} A^{1}+\operatorname{det} A^{2}+\operatorname{det} A^{3}\right)
$$

where

$$
A^{1}=\left(\begin{array}{cc}
\partial_{1} \eta_{\mu} \partial_{1} \mathcal{E} v^{\mu} & \partial_{1} \eta_{\mu} \partial_{2} \\
\partial_{2} \eta_{\mu} \partial_{1} \mathcal{E} v^{\mu} & \partial_{2} \eta_{\mu} \partial_{2} \mathcal{E} v^{\mu}
\end{array}\right), A^{2}=\left(\begin{array}{cc}
\partial_{1} v_{\mu} \partial_{1} \mathcal{E} v^{\mu} & \partial_{1} \eta_{\mu} \partial_{2} \mathcal{E} v^{\mu} \\
\partial_{2} v_{\mu} \partial_{1} \mathcal{E} v^{\mu} & \partial_{2} \eta_{\mu} \partial_{2} \mathcal{E} v^{\mu}
\end{array}\right), A^{3}=\left(\begin{array}{cc}
\partial_{1} \eta_{\mu} \partial_{1} \mathcal{E} v^{\mu} & \partial_{1} v_{\mu} \partial_{2} \mathcal{E} v^{\mu} \\
\partial_{2} \eta_{\mu} \partial_{1} \mathcal{E} v^{\mu} & \partial_{2} v_{\mu} \partial_{2} \mathcal{E} v^{\mu}
\end{array}\right)
$$


and we obtain

$$
\begin{aligned}
I_{421} & =-\int_{\Gamma_{1}} \partial_{t}\left(\frac{\bar{\theta}}{\sqrt{g}} \operatorname{det} A^{1}\right)+\int_{\Gamma_{1}} \partial_{t}\left(\frac{\bar{\theta}}{\sqrt{g}}\right) \operatorname{det} A^{1}-\int_{\Gamma_{1}} \frac{\bar{\theta}}{\sqrt{g}} \operatorname{det} A^{2}-\int_{\Gamma_{1}} \frac{\bar{\theta}}{\sqrt{g}} \operatorname{det} A^{3} \\
& =I_{4211}+I_{4212}+I_{4213}+I_{4214} .
\end{aligned}
$$

As above,

$$
\left\|\partial_{t}(\bar{\theta} / \sqrt{g})\right\|_{L^{\infty}\left(\Gamma_{1}\right)} \lesssim P\left(\|\eta\|_{H^{2.5+\delta_{0}}}\right)\|v\|_{H^{2.5+\delta_{0}}}
$$

and $\left|\operatorname{det} A^{1}\right| \lesssim|\bar{\partial} \eta|^{2}(\mathcal{E} \bar{\partial} v)^{2}$. Therefore,

$$
I_{4212} \lesssim P\left(\|\eta\|_{H^{2.5+\delta_{0}}}\right)\|v\|_{H^{2.5+\delta_{0}}}\|\mathcal{E} \bar{\partial} v\|_{L^{2}\left(\Gamma_{1}\right)}^{2} \lesssim P\left(\|\eta\|_{H^{2.5+\delta_{0}}}\right)\|v\|_{H^{2.5+\delta_{0}}}\|\mathcal{E} v\|_{H^{1.5}}^{2}
$$

as well as

$$
\left|\int_{\Gamma_{1}} \frac{1}{\sqrt{g}}\left(\operatorname{det} A^{2}+\operatorname{det} A^{3}\right)\right| \lesssim P\left(\|\eta\|_{H^{2.5+\delta_{0}}}\right)\|\mathcal{E} \bar{\partial} v\|_{L^{2}\left(\Gamma_{1}\right)}^{2} \lesssim P\left(\|\eta\|_{H^{2.5+\delta_{0}}}\right)\|\mathcal{E} v\|_{H^{1.5}}^{2} .
$$

The rest is the same as in Section 6. We integrate by parts and write

$$
\begin{aligned}
\int_{\Gamma_{1}} \frac{\bar{\theta}}{\sqrt{g}} \operatorname{det} A^{1} & =\int_{\Gamma_{1}} \frac{\bar{\theta}}{\sqrt{g}}\left(\partial_{1} \eta_{\mu} \partial_{2} \eta_{\lambda} \partial_{1} \mathcal{E} v^{\mu} \partial_{2} \mathcal{E} v^{\lambda}-\partial_{1} \eta_{\mu} \partial_{2} \eta_{\lambda} \partial_{2} \mathcal{E} v^{\mu} \partial_{1} \mathcal{E} v^{\lambda}\right) \\
& =\int_{\Gamma_{1}} \frac{\bar{\theta}}{\sqrt{g}}\left(-\partial_{1} \eta_{\mu} \partial_{2} \eta_{\lambda} \mathcal{E} v^{\mu} \partial_{1} \partial_{2} \mathcal{E} v^{\lambda}+\partial_{1} \eta_{\mu} \partial_{2} \eta_{\lambda} \mathcal{E} v^{\mu} \partial_{2} \partial_{1} \mathcal{E} v^{\lambda}\right)-\int_{\Gamma_{1}} \bar{\theta} Q_{\mu \lambda}^{i}\left(\bar{\partial} \eta, \bar{\partial}^{2} \eta\right) \mathcal{E} v^{\mu} \partial_{i} \mathcal{E} v^{\lambda} \\
& =-\int_{\Gamma_{1}} \bar{\theta} Q_{\mu \lambda}^{i}\left(\bar{\partial} \eta, \bar{\partial}^{2} \eta\right) \mathcal{E} v^{\mu} \partial_{i} \mathcal{E} v^{\lambda}
\end{aligned}
$$

where $Q_{\mu \lambda}^{i}\left(\bar{\partial} \eta, \bar{\partial}^{2} \eta\right)$ is a rational function, which is linear in $\bar{\partial}^{2} \eta$. Therefore,

$$
I_{4211}=\frac{d}{d t} \int_{\Gamma_{1}} \bar{\theta} \tilde{Q}_{\mu \lambda}^{i}(\bar{\partial} \eta) \bar{\partial}^{2} \eta \mathcal{E} v^{\mu} \partial_{i} \mathcal{E} v^{\lambda}
$$

and we obtain

$$
I_{421} \lesssim \frac{d}{d t} \int_{\Gamma_{1}} \bar{\theta} \tilde{Q}_{\mu \lambda}^{i}(\bar{\partial} \eta) \bar{\partial}^{2} \eta \mathcal{E} v^{\mu} \partial_{i} \mathcal{E} v^{\lambda}+P\left(\|\eta\|_{H^{2.5+\delta_{0}}}\right)\left(\|v\|_{H^{2.5+\delta_{0}}}+1\right)\|\mathcal{E} v\|_{H^{1.5}}
$$

Thus we have shown how to adapt the result in Section 7 to the case of the curved domain.

After covering $\Gamma$ with finitely many balls $\left\{B_{r_{\ell}}\left(y_{\ell}\right)\right\}_{\ell=0}^{N}$, the procedure described above yields the desired estimates near the boundary. In order to obtain the full estimate, we need to bound the solution in the region of $\Omega$ not covered by $B=\bigcup_{\ell=0}^{N} B_{r_{\ell}}\left(y_{\ell}\right)$. This is done by covering $\Omega \backslash B$ with further open sets and again reducing the problem to estimates on $\mathbb{T}^{2} \times[0,1]$. However, for these estimates no integrals on either $\Gamma_{1}$ or $\Gamma_{2}$ will appear.

Using again cut-off functions and the local parameterization of $\Omega$ described above, the $L^{2}$ estimate for $\partial_{t}^{2} v$ in Section 8 is easily adapted to the present situation since only an integer number of derivatives is used in those estimates. The later sections, including the div-curl estimates and the Cauchy invariance property, are also easily adaptable. This establishes Theorems 12.1 and 12.2, except for the statement $\|\Gamma(t)\|_{H^{3+\epsilon}} \leq C_{*}$, which we now prove.

Let $y_{0} \in \eta(\Omega)$. We choose coordinates $\left(y^{1}, y^{2}, y^{3}\right)$ in the ambient Euclidean space such that, possibly after a rigid motion and relabeling of the coordinates, $y_{0}$ is identified with the origin and $\eta(\Omega)$ is locally given by a graph $y^{3}=h\left(y^{1}, y^{2}\right)$. Denote by $\Sigma$ the portion of $\eta(\Omega)$ that is written as the graph of $h$. We can further assume that $\partial_{y^{i}}$, for $i=1,2$, are tangent to $\Sigma$ at $y_{0}=(0,0,0)$ and that $\partial_{y^{1}} h\left(y_{0}\right)=\partial_{y^{2}} h\left(y_{0}\right)=0$.

Recall that we denote by $\mathcal{H}: \eta(\Omega) \rightarrow \mathbb{R}$ the mean curvature of $\eta(\Omega)$. In terms of local coordinates $\left(x^{1}, x^{2}, x^{3}\right)$ near $\eta^{-1}\left(y_{0}\right)$ we have the known formula $-\Delta_{g} \eta^{\alpha}=\mathcal{H} \circ \eta n^{\alpha} \circ \eta$, where $n$ is the unit outer normal to $\eta(\Omega)$. Contracting with $n^{\alpha} \circ \eta$, invoking (2.6) and (12.1) (which is the part of Theorem 12.1 that has already been established) 
we have $\|\mathcal{H}\|_{H^{1+\epsilon}} \leq C_{*}$ (here, and in what follows, we relabel the constant $C_{*}$ if necessary). On the other hand, setting $w=h-y^{3}$, we have the following expression for the mean curvature expressed in $y$-coordinates:

$$
A^{i j}(\nabla w) \partial_{i} \partial_{j} w=\frac{1}{|\nabla w|}\left(\delta^{i j}-\frac{\partial^{i} w \partial^{j} w}{|\nabla w|^{2}}\right) \partial_{i} \partial_{j} w=\mathcal{H} \circ h,
$$

where $\partial^{i}=\delta^{i k} \partial_{k}$. From the way we constructed $h$, we have $A^{i j}\left(y_{0}\right)=\delta^{i j}$. We already know that $\|\Sigma\|_{H^{2+\epsilon}} \leq C_{*}$ since we have a bound for $\eta$, thus we may assume that $\|w\|_{2+\epsilon} \leq C_{*}$. It follows that $A^{i j}$ is uniformly elliptic near the origin and bounded in $C^{0, \beta}$ for some $0<\beta<\epsilon$. Elliptic regularity then implies that $\|w\|_{H^{3+\epsilon}} \leq C_{*}$, as desired.

We remark that the application of elliptic theory in the previous paragraph is not entirely immediate, and has to be carried out in steps due to the low regularity of the coefficients $A^{i j}$. First, one uses Schauder theory and the embedding $H^{1+\epsilon}(\Sigma) \subset C^{0, \beta}(\Sigma)$ to conclude that $w$ is in $C^{2, \beta}$. Then the coefficients $A^{i j}$ are in fact $C^{1, \beta}$. Using that the right hand side of (12.4) is in $H^{1}$ we can then apply $L^{p}$ estimates to obtain $w \in H^{3}$. Thus, $A^{i j}$ is now in $H^{2}$, and we can interpolate between estimates for elliptic operators with coefficients in Sobolev spaces of integer order to finally conclude the result.

\section{REFERENCES}

[1] T. Alazard. About global existence and asymptotic behavior for two dimensional gravity water waves. In Séminaire Laurent SchwartzÉquations aux dérivées partielles et applications. Année 2012-2013, Sémin. Équ. Dériv. Partielles, pages Exp. No. XVIII, 16. École Polytech., Palaiseau, 2014.

[2] T. Alazard. Stabilization of the Water-Wave Equations with Surface Tension. Ann. PDE, 3(2):3:17, 2017.

[3] T. Alazard and P. Baldi. Gravity capillary standing water waves. Arch. Ration. Mech. Anal., 217(3):741-830, 2015.

[4] T. Alazard, N. Burq, and C. Zuily. On the water-wave equations with surface tension. Duke Math. J., 158(3):413-499, 2011.

[5] T. Alazard, N. Burq, and C. Zuily. Strichartz estimates for water waves. Ann. Sci. Éc. Norm. Supér. (4), 44(5):855-903, 2011.

[6] T. Alazard, N. Burq, and C. Zuily. Low regularity Cauchy theory for the water-waves problem: canals and wave pools. In Lectures on the analysis of nonlinear partial differential equations. Part 3, volume 3 of Morningside Lect. Math., pages 1-42. Int. Press, Somerville, MA, 2013.

[7] T. Alazard, N. Burq, and C. Zuily. The water-wave equations: from Zakharov to Euler. In Studies in phase space analysis with applications to PDEs, volume 84 of Progr. Nonlinear Differential Equations Appl., pages 1-20. Birkhäuser/Springer, New York, 2013.

[8] T. Alazard, N. Burq, and C. Zuily. On the Cauchy problem for gravity water waves. Invent. Math., 198(1):71-163, 2014.

[9] T. Alazard, N. Burq, and C. Zuily. Cauchy theory for the gravity water waves system with non-localized initial data. Ann. Inst. H. Poincaré Anal. Non Linéaire, 33(2):337-395, 2016.

[10] T. Alazard and J.-M. Delort. Global solutions and asymptotic behavior for two dimensional gravity water waves. Ann. Sci. Éc. Norm. Supér. (4), 48(5):1149-1238, 2015.

[11] D. M. Ambrose. Well-posedness of vortex sheets with surface tension. SIAM J. Math. Anal., 35(1):211-244 (electronic), 2003.

[12] D. M. Ambrose and N. Masmoudi. The zero surface tension limit of two-dimensional water waves. Comm. Pure Appl. Math., 58:1287-1315, 2005.

[13] J. T. Beale, T. Y. Hou, and J. S. Lowengrub. Growth rates for the linearized motion of fluid interfaces away from equilibrium. Comm. Pure Appl. Math., 46(9):1269-1301, 1993.

[14] Lydia Bieri, Shuang Miao, Sohrab Shahshahani, and Sijue Wu. On the Motion of a Self-Gravitating Incompressible Fluid with Free Boundary. Comm. Math. Phys., 355(1):161-243, 2017.

[15] Jean Bourgain and Dong Li. Strong ill-posedness of the incompressible Euler equation in borderline Sobolev spaces. Invent. Math., 201(1):97-157, 2015.

[16] J. P. Bourguignon and H. Brezis. Remarks on the Euler equation. Journal of Functional Analysis, 15:341-363, 1974.

[17] A. Castro, D. Córdoba, C. Fefferman, F. Gancedo, and J. Gómez-Serrano. Finite time singularities for water waves with surface tension. J. Math. Phys., 53(11):115622, 26, 2012.

[18] A. Castro, D. Córdoba, C. Fefferman, F. Gancedo, and J. Gómez-Serrano. Finite time singularities for the free boundary incompressible Euler equations. Ann. of Math. (2), 178(3):1061-1134, 2013.

[19] A. Castro, D. Córdoba, C. Fefferman, F. Gancedo, and J. Gómez-Serrano. Structural stability for the splash singularities of the water waves problem. Discrete Contin. Dyn. Syst., 34(12):4997-5043, 2014.

[20] A. Castro, D. Córdoba, C. Fefferman, F. Gancedo, and M. López-Fernández. Rayleigh-Taylor breakdown for the Muskat problem with applications to water waves. Ann. of Math. (2), 175(2):909-948, 2012.

[21] C.-H. A. Chen, D. Coutand, and S. Shkoller. Solvability and regularity for an elliptic system prescribing the curl, divergence, and partial trace of a vector field on Sobolev-class domains. J. Math. Fluid Mech., 19(3):375-422, 2017.

[22] Ching-Hsiao Arthur Cheng, Daniel Coutand, and Steve Shkoller. On the motion of vortex sheets with surface tension in three-dimensional Euler equations with vorticity. Comm. Pure Appl. Math., 61(12):1715-1752, 2008.

[23] D. Christodoulou and H. Lindblad. On the motion of the free surface of a liquid. Comm. Pure Appl. Math., 53(12):1536-1602, 2000.

[24] Peter Constantin and Ciprian Foias. Navier-Stokes equations. Chicago Lectures in Mathematics. University of Chicago Press, Chicago, IL, 1988. 
[25] D. Coutand and S. Shkoller. Well-posedness of the free-surface incompressible Euler equations with or without surface tension. J. Amer. Math. Soc., 20(3):829-930, 2007.

[26] D. Coutand and S. Shkoller. A simple proof of well-posedness for the free-surface incompressible Euler equations. Discrete Contin. Dyn. Syst. Ser. S, 3(3):429-449, 2010.

[27] D. Coutand and S. Shkoller. On the finite-time splash and splat singularities for the 3-D free-surface Euler equations. Commun. Math. Phys., 325:143-183, 2014.

[28] Daniel Coutand. Finite-time singularity formation for incompressible Euler moving interfaces in the plane. Arch. Ration. Mech. Anal., 232(1):337-387, 2019.

[29] W. Craig. On the Hamiltonian for water waves. arXiv:1612.08971 [math.AP], page 10 pages, 2016.

[30] Thibault de Poyferré. A priori estimates for water waves with emerging bottom. Arch. Ration. Mech. Anal., 232(2):763-812, 2019.

[31] Yu Deng, Alexandru D. Ionescu, Benoît Pausader, and Fabio Pusateri. Global solutions of the gravity-capillary water-wave system in three dimensions. Acta Math., 219(2):213-402, 2017.

[32] M. M. Disconzi. On a linear problem arising in dynamic boundaries. Evol. Equ. Control Theory, 3(4):627-644, 2014.

[33] M. M. Disconzi and D. G. Ebin. On the limit of large surface tension for a fluid motion with free boundary. Comm. Partial Differential Equations, 39(4):740-779, 2014.

[34] M. M. Disconzi and D. G. Ebin. The free boundary Euler equations with large surface tension. Journal of Differential Equations, 261(2):821-889, 2016.

[35] M. M. Disconzi and I. Kukavica. A priori estimates for the free-boundary Euler equations with surface tension in three dimensions. arXiv: 1708.00086 [math.AP], 2017. 40 pages.

[36] D. G. Ebin. The equations of motion of a perfect fluid with free boundary are not well posed. Comm. Partial Differential Equations, 12(10):1175-1201, 1987.

[37] D. G. Ebin and J. Marsden. Groups of diffeomorphisms and the motion of an incompressible fluid. Annals of Math, 92:102-163, 1970.

[38] Lawrence C. Evans. Partial differential equations, volume 19 of Graduate Studies in Mathematics. American Mathematical Society, Providence, RI, second edition, 2010.

[39] C. Fefferman, A. D. Ionescu, and V. Lie. On the absence of splash singularities in the case of two-fluid interfaces. Duke Math. J., 165(3):417$462,2016$.

[40] P. Germain, N. Masmoudi, and J. Shatah. Global solutions for the gravity water waves equation in dimension 3. Ann. of Math. (2), 175(2):691-754, 2012.

[41] P. Germain, N. Masmoudi, and J. Shatah. Global existence for capillary water waves. Comm. Pure Appl. Math., 68(4):625-687, 2015.

[42] Loukas Grafakos and Seungly Oh. The Kato-Ponce inequality. Comm. Partial Differential Equations, 39(6):1128-1157, 2014.

[43] J. K Hunter, M. Ifrim, and D. Tataru. Two dimensional water waves in holomorphic coordinates. Comm. Math. Phys., 346(2):483-552, 2016.

[44] M. Ifrim and D. Tataru. Two dimensional water waves in holomorphic coordinates II: Global solutions. Bull. Soc. Math. France, 144(2):369394, 2016.

[45] M. Ifrim and D. Tataru. The Lifespan of Small Data Solutions in Two Dimensional Capillary Water Waves. Arch. Ration. Mech. Anal., 225(3):1279-1346, 2017.

[46] Mihaela Ifrim and Daniel Tataru. Two-dimensional gravity water waves with constant vorticity I: Cubic lifespan. Anal. PDE, 12(4):903-967, 2019.

[47] M. Ignatova and I. Kukavica. On the local existence of the free-surface Euler equation with surface tension. Asymptot. Anal., 100(1-2):63$86,2016$.

[48] T. Iguchi. Well-posedness of the initial value problem for capillary-gravity waves. Funkcial. Ekvac., 44(2):219-241, 2001.

[49] T. Iguchi, N. Tanaka, and A. Tani. On a free boundary problem for an incompressible ideal fluid in two space dimensions. Adv. Math. Sci. Appl., 9(1):415-472, 1999.

[50] A. D. Ionescu and F. Pusateri. Global solutions for the gravity water waves system in 2d. Invent. Math., 199(3):653-804, 2015.

[51] A. D. Ionescu and F. Pusateri. Global analysis of a model for capillary water waves in two dimensions. Comm. Pure Appl. Math., 69(11):2015-2071, 2016.

[52] Alexandru D. Ionescu and Fabio Pusateri. Global regularity for 2D water waves with surface tension. Mem. Amer. Math. Soc., 256(1227): $\mathrm{v}+124,2018$.

[53] Tosio Kato and Gustavo Ponce. Well-posedness of the Euler and Navier-Stokes equations in the Lebesgue spaces $L_{s}^{p}\left(\mathbf{R}^{2}\right) . R e v . M a t$. Iberoamericana, 2(1-2):73-88, 1986.

[54] Carlos E. Kenig, Gustavo Ponce, and Luis Vega. On the (generalized) Korteweg-de Vries equation. Duke Math. J., 59(3):585-610, 1989.

[55] Carlos E. Kenig, Gustavo Ponce, and Luis Vega. Well-posedness of the initial value problem for the Korteweg-de Vries equation. J. Amer. Math. Soc., 4(2):323-347, 1991.

[56] I. Kukavica and A. Tuffaha. On the 2D free boundary Euler equation. Evol. Equ. Control Theory, 1(2):297-314, 2012.

[57] I. Kukavica and A. Tuffaha. A regularity result for the incompressible Euler equation with a free interface. Appl. Math. Optim., 69(3):337$358,2014$.

[58] Igor Kukavica, Amjad Tuffaha, and Vlad Vicol. On the local existence and uniqueness for the 3D Euler equation with a free interface. Appl. Math. Optim., 76(3):535-563, 2017.

[59] Igor Kukavica, Fei Wang, and Mohammed Ziane. Persistence of regularity for solutions of the Boussinesq equations in Sobolev spaces. Adv. Differential Equations, 21(1-2):85-108, 2016.

[60] D. Lannes. Well-posedness of the water-waves equations. J. Amer. Math. Soc., 18(3):605-654 (electronic), 2005. 
[61] D. Lannes. The water waves problem, volume 188 of Mathematical Surveys and Monographs. American Mathematical Society, Providence, RI, 2013. Mathematical analysis and asymptotics.

[62] H. Lindblad. The motion of the free surface of a liquid. In Séminaire: Équations aux Dérivées Partielles, 2000-2001, Sémin. Équ. Dériv. Partielles, pages Exp. No. VI, 10. École Polytech., Palaiseau, 2001.

[63] H. Lindblad. Well-posedness for the linearized motion of an incompressible liquid with free surface boundary. Comm. Pure Appl. Math., 56(2):153-197, 2003

[64] H. Lindblad and K. H. Nordgren. A priori estimates for the motion of a self-gravitating incompressible liquid with free surface boundary. J. Hyperbolic Differ. Equ., 6(2):407-432, 2009.

[65] V. I. Nalimov. The Cauchy-Poisson problem. Dinamika Splošn. Sredy, (Vyp. 18 Dinamika Zidkost. so Svobod. Granicami):104-210, 254, 1974.

[66] M. Ogawa and A. Tani. Free boundary problem for an incompressible ideal fluid with surface tension. Math. Models Methods Appl. Sci., 12(12):1725-1740, 2002.

[67] M. Ogawa and A. Tani. Incompressible perfect fluid motion with free boundary of finite depth. Adv. Math. Sci. Appl., 13(1):201-223, 2003.

[68] F. Pusateri. On the limit as the surface tension and density ratio tend to zero for the two-phase Euler equations. J. Hyperbolic Differ. Equ., 8(2):347-373, 2011.

[69] B. Schweizer. On the three-dimensional Euler equations with a free boundary subject to surface tension. Ann. Inst. H. Poincaré Anal. Non Linéaire, 22(6):753-781, 2005.

[70] J. Shatah and C. Zeng. Geometry and a priori estimates for free boundary problems of the Euler equation. Comm. Pure Appl. Math., 61(5):698-744, 2008.

[71] J. Shatah and C. Zeng. Local well-posedness for fluid interface problems. Arch. Ration. Mech. Anal., 199(2):653-705, 2011.

[72] Roger Temam. Navier-Stokes equations. AMS Chelsea Publishing, Providence, RI, 2001. Theory and numerical analysis, Reprint of the 1984 edition.

[73] C. Wang, Z. Zhang, W. Zhao, and Y. Zheng. Local well-posedness and break-down criterion of the incompressible euler equations with free boundary. arXiv:1507.02478, 2015

[74] S. Wu. Well-posedness in Sobolev spaces of the full water wave problem in 2-D. Invent. Math., 130(1):39-72, 1997.

[75] S. Wu. Well-posedness in Sobolev spaces of the full water wave problem in 3-D. J. Amer. Math. Soc., 12(2):445-495, 1999.

[76] S. Wu. Almost global wellposedness of the 2-D full water wave problem. Invent. Math., 177(1):45-135, 2009.

[77] S. Wu. Global wellposedness of the 3-D full water wave problem. Invent. Math., 184(1):125-220, 2011.

[78] H. Yosihara. Gravity waves on the free surface of an incompressible perfect fluid of finite depth. Publ. Res. Inst. Math. Sci., 18(1):49-96, 1982.

[79] H. Yosihara. Capillary-gravity waves for an incompressible ideal fluid. J. Math. Kyoto Univ., 23(4):649-694, 1983.

Department of Mathematics, VAnderbilt University, NashVille, TN, USA

E-mail address: marcelo.disconzi@vanderbilt.edu

Department of Mathematics, University of Southern California, Los Angeles, CA 90089

E-mail address: kukavica@usc.edu

Department of Mathematics and Statistics, American University of SharJah, SharJah, UAE

E-mail address: atufaha@aus.edu 